\title{
Multi-wavelength Observations of Coronal Waves and Oscillations in Association with Solar Eruptions
}

\author{
Dissertation \\ zur Erlangung des Doktorgrades \\ der Mathematisch-Naturwissenschaftlichen Fakultäten \\ der Georg-August-Universität zu Göttingen
}

vorgelegt von

Danica Tóthová

aus Bratislava, Slowakei

Göttingen 2010 
D7

Referent: Prof. Dr. Kneer

Korreferent: Prof. Dr. Solanki

Tag der mündlichen Prüfung: 


\section{Contents}

\begin{tabular}{lr}
\hline Summary & 8
\end{tabular}

$\begin{array}{lll}1 & \text { Introduction } & 9\end{array}$

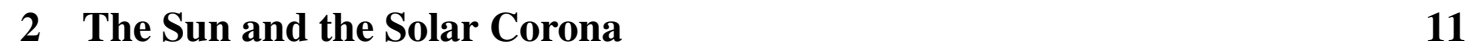

2.1 The Sun and its Atmosphere $\ldots \ldots \ldots \ldots \ldots$

2.2 The Corona . . . . . . . . . . . . . . . . . . . . . . . 14

2.2.1 Emission Measure and Differential Emission Measure . . . . . 16

2.2 .2 Continuum Spectrum of the Corona . . . . . . . . . . . 17

2.2.2.1 Free-free Continuum . . . . . . . . . . . . . . . 17

2.2 .2 .2 Free-bound Continuum . . . . . . . . . . . . . . . 19

2.2 .2 .3 Two-photon continuum . . . . . . . . . . . . . . 19

2.2.2.4 Synthetic CHIANTI Continuum Spectrum . . . . . . 20

2.2 .3 Emission Lines . . . . . . . . . . . . . . . . . . . . . . . . 21

2.2.3.1 EUV Emission Line Spectroscopy . . . . . . . . . . 21

2.2 .3 .2 Line Characteristics . . . . . . . . . . . . . . 23

2.2.3.3 Synthetic CHIANTI Emission Line Spectrum . . . . 24

2.2 .3 .4 SUMER EUV Spectrum $\ldots \ldots \ldots \ldots \ldots \ldots$

\begin{tabular}{|lll}
\hline 3 & Magnetohydrodynamics & 27
\end{tabular}

3.1 MHD Equations . . . . . . . . . . . . . . . . . . . . . . . . 27

3.2 Dimensionless Plasma Parameters . . . . . . . . . . . . . . . 29

3.3 Ideal Adiabatic MHD Equations $\ldots \ldots \ldots \ldots \ldots$

3.3 .1 Force-free Equilibrium . . . . . . . . . . . . . . . . . 32

3.4 Resistive MHD . . . . . . . . . . . . . . . . . . . 34

3.4.1 MHD in the diffusive limit . . . . . . . . . . . . . . 34

\begin{tabular}{lll}
\hline 4 & Solar Eruptions & 37
\end{tabular}

$4.1 \quad$ Magnetic Reconnection . . . . . . . . . . . . . . . . . . . . . . . 37

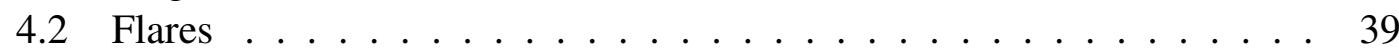

$4.2 .1 \quad$ Flare Emission . . . . . . . . . . . . . . . . 40 
$4.2 .1 .1 \quad \gamma$-Rays and X-Rays . . . . . . . . . . . 40

4.2.1.2 Flare Evolution in $\gamma$ and X-Rays . . . . . . . . . . 42

4.2 .1 .3 Ultraviolet (UV) Emission . . . . . . . . . . . . . . 46

4.2 .1 .4 Radio Emission . . . . . . . . . . . . . . . . 46

4.3 Eruptive Prominences $\ldots \ldots \ldots$. . . . . . . . . . . . . . . . . . . . . 49

4.3 .1 EUV Observations of Prominences . . . . . . . . . . . 50

4.4 Coronal Mass Ejections . . . . . . . . . . . . . . . . . . . . 51

4.4 .1 CME Observations . . . . . . . . . . . . . . . . 51

4.4 .2 Theoretical Models of CME Initiation . . . . . . . . . . . 52

$4.4 .2 .1 \quad$ Pre-Eruptive Topology . . . . . . . . . . . . 52

$4.4 .2 .2 \quad$ Magnetic Flux-Rope Model . . . . . . . . . . . . . 54

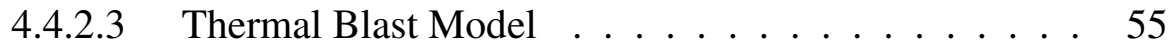

4.4 .2 .4 Dynamo Model . . . . . . . . . . . . . 56

4.4 .2 .5 Break-out Model . . . . . . . . . . . . . . . . . . 56

$\begin{array}{llr}5 & \text { Coronal Waves and Oscillations } & 59\end{array}$

5.1 Introduction . . . . . . . . . . . . . . . . . . . 59

5.2 Theoretical Overview . . . . . . . . . . . . . . . . . 59

5.2.1 Definition of Characteristic Phase Speeds of MHD Waves . . . 60

5.2.2 MHD Waves in an Unbounded Homogeneous Medium . . . . . 61

$5.2 .2 .1 \quad$ Special solutions . . . . . . . . . . . . 62

5.2 .2 .2 General solutions . . . . . . . . . . . . 63

5.2 .3 Waves in a Straight Magnetic Cylinder . . . . . . . . . . . 64

5.2 .4 Alfvén Waves . . . . . . . . . . . . . . . . . . . 67

5.2 .5 Sausage Mode and Kink Mode . . . . . . . . . . . . . . . . 67

5.2 .6 Slow Magneto-acoustic Waves . . . . . . . . . . . . . . . . 68

5.3 Non-Ideal MHD Effects . . . . . . . . . . . . . . . . . . . . . . . . . . 69

6 Multi-wavelength Observations of Oscillations in the Wake of a Flare Blast

\begin{tabular}{lc}
\hline Wave & 71
\end{tabular}

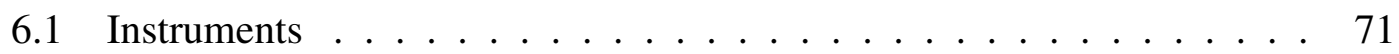

6.1.1 Solar Ultraviolet Measurements of Emitted Radiation (SUMER) on board of Solar Heliospheric Observatory (SoHO) 71

6.1 .2 Transition Region And Coronal Explorer (TRACE) . . . . . . . 72

$6.1 .3 \quad$ Meudon Heliograph $\ldots \ldots \ldots$. . . . . . . . 72

6.2 Wavelet Analysis . . . . . . . . . . . . . . . . . . 74

6.2 .1 Time Series Analysis . . . . . . . . . . . . . . . . 75

6.2.1.1 Continuous Wavelet Transform . . . . . . . . . . 75

6.2 .2 Image Enhancement - Unsharp Masking . . . . . . . . . . . 77

6.3 9 April 2002 C Class Flare . . . . . . . . . . . . . . . . . 80

6.3 .1 Observations . . . . . . . . . . . . . . . . 80 
6.3 .1 .1 TRACE ..................... 82

6.3 .1 .2 Meudon Heliograph $\ldots \ldots \ldots$. . . . . . . 83

6.3 .1 .3 SUMER . . . . . . . . . . . . . . . 83

6.3 .2 Ejecta, Fronts and Oscillations . . . . . . . . . . . . 84

6.4 16 April 2002 M Class Flare $\ldots \ldots \ldots$. . . . . . . . . . . . . . . 89

6.4 .1 Introduction . . . . . . . . . . . . . . . . . . 89

6.4 .2 Observations . . . . . . . . . . . . . . . . 90

6.5 Discussion and Conclusions $\ldots \ldots \ldots$. . . . . . . . . . 98

\begin{tabular}{lll}
\hline 7 & Outlook & 101
\end{tabular}

\begin{tabular}{lll}
\hline A Appendix & 103 \\
\hline
\end{tabular}

A.1 Notation . . . . . . . . . . . . . . . . . . . . . 103

A.1.1 Quantities . . . . . . . . . . . . . . . 103

A.1.2 Constants . . . . . . . . . . . . . . . . . . . . 106

A.2 Spectral Notation . . . . . . . . . . . . . . . . . . 107

A.3 Line List . . . . . . . . . . . . . . . . . . . . . . . . . . . . . . . . . . 108

A.3.1 SUMER Range . . . . . . . . . . . . . . . . . . . . . 108

A.3.2 TRACE Range . . . . . . . . . . . . . . . . . . . . . 109

A.4 Radiative Transfer . . . . . . . . . . . . . . . . . . . . . . . . . . . 110

A.5 Local Thermodynamic Equilibrium . . . . . . . . . . . . . . . 111

A.5.1 Maxwellian Distribution . . . . . . . . . . . . . . . 111

A.5.2 Boltzmann equation . . . . . . . . . . . . . . 112

A.5.3 Saha Ionization Equation . . . . . . . . . . . . . . . . . . 112

A.5.4 Planck Function . . . . . . . . . . . . . . . . . . . . . 112

A.6 Fourier Transform . . . . . . . . . . . . . . . . . . 113

\begin{tabular}{ll}
\hline Bibliography & 115
\end{tabular}

\begin{tabular}{ll}
\hline Publications & 121
\end{tabular}

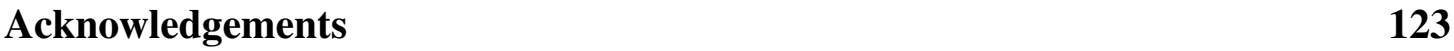

\begin{tabular}{ll}
\hline Curriculum Vitae & 125
\end{tabular} 



\section{Summary}

This thesis presents an observational study of the relationship between flares and filament eruptions, and associated waves and oscillations in flare loops and ambient corona. A detailed analysis of the oscillations seen in flare loops and the ambient corona in both TRACE and SUMER spectra is shown. We argue, that although the flares are associated with slowly rising EUV loop and prominence eruptions, the oscillations were significant in a wide region along the slit far beyond the edge of the rising loops. We suggest an interpretation in terms of heating and acceleration of plasma in the wake of a flare blast wave.

\section{Analysis of the 9 April flare-CME event}

- A power map of lowest frequency intensity variations in the pre-flare phase has been constructed revealing flows along the TRACE loops and the prominence.

- Doppler shifts were calculated from the observations from Meudon and SUMER. A detailed inspection of SUMER spectra was performed in order to prevent a false interpretation of the emission in wings du to contamination from the blends. Only the actual Doppler shifts were used in the analysis.

- The coalignment of the SUMER and TRACE observations was made based on the common features in early pre-flare stage with the help of the common features in SUMER and Meudon observations after the flare.

- A movie was constructed showing the relationship of the observations in different wavelengths.

- We have shown a front with a plane-of-sky velocity $250 \mathrm{~km} \mathrm{~s}^{-1}$ ahead of the erupting filament in TRACE $195 \AA$. It was followed by an enhanced emission in $\mathrm{Si}$ III , brief red shifted bursts moving south and north along the slit, and finally by the rapid decrease in Si III intensity. At the same time, the intensity of the flare line Fe XIX increased and a Doppler shift oscillation lasting at least 1 hour was launched along broad sections of the slit, with a varying amplitude and period. 
- Wavelet analysis have revealed three different oscillations detected with SUMER and TRACE. Based on the observed periods, we have identified a standing magnetoacoustic mode with a 14 minute period in the latitudes 50" $<y<100$ " and $150 "<y<180 "$. In the northern section, a kink oscillation with a 4 min period was revealed. A 7 min displacement oscillation also attributed to the kink mode has been observed further out in the corona. All the oscillations had a high initial pulse supporting the idea of an impulsive trigger.

The re-analysis of the 16 April flare-CME event

- The coalignment of the SUMER and TRACE observations was made based on the common features.

- A movie was constructed showing the relationship of the observations in different wavelengths.

- We have revealed long lasting large red and blue Doppler shifts prior to the eruption of the filament. These were previously interpreted by Wang et al. (2007) as reconnection outflows based on CSHKP model.

- We suggested, that the above mentioned shifts could not be interpreted solely in terms of reconnection jets based on the large width of the region of their occurrence. According to the CSHKP model, reconnection outflows would be confined to a narrow region.

- An oscillation of the Fe XIx line and an intensity oscillation of $195 \AA$ emission was seen during and after the filament eruption. Based on the fact that it spread far beyond the legs of the rising prominence, we suggested it was triggered by the blast wave. 


\section{Introduction}

Modern imaging and spectral instruments allow observations of waves and oscillations (i.e. standing waves) in visible, X-ray, EUV and radio wavelengths in the solar corona. They are interpreted in terms of magnetohydrodynamic theory and modeled with the help of numerical simulations. Three basic magneto-acoustic modes of waves can be sustained by the compressible, elastic corona: fast, slow (sound) and intermediate (also called pure Alfvén).

Trigger mechanism of the waves involves deviation from the magnetohydrostatic equilibrium, a perturbation often induced by solar flares, and are sustained due to the mutually restoring forces acting in opposite directions. Due to the complex magnetic structures in the corona, these modes interact and their study itself is an interesting physics problem. The role of the coronal waves in coronal heating due to the damping of the oscillations by resonant absorption has been studied by Poedts et al. (1989), Aschwanden (2004), Nakariakov et al. (1999). Understanding of waves is also motivated by the possibility of diagnostic of the plasma through the observations of perturbed macroscopic parameters by means of coronal seismology suggested by Roberts (1983).

TRACE imaging observations revealed displacement oscillations of coronal loops, which were interpreted in terms of flare-triggered kink oscillations (Aschwanden et al. 1999. 2002, Nakariakov et al. 1999, Schrijver et al. 2002). Spectroscopic observations have discovered strongly damped quasi-periodic oscillations of the Doppler shift of the flare lines Fe XIX and Fe XXI (Kliem et al. 2002, Wang et al. 2002). SUMER spectroscopic observations allow the detection of density perturbations seen as intensity fluctuations of the coronal lines. In the rare cases where both intensity and Doppler shift oscillation have been seen, the phase shift between the two implies longitudinal slow mode waves. Wave-mode recognition depends critically on the accuracy of determination of the geometry of the studied oscillating region. If the perturbations have a line-of-sight component, they can be seen as Doppler shift fluctuations. These observations have been made with a slit spectrometer at a single position (Ofman \& Wang 2002, Wang et al. 2002, 2003b, 2005). Context images of the segment covered by the spectrometer slit are essential for giving information on the plane-of-sky velocity component which can help reveal the geometry of the events, find the timing of associated events, link the oscillating structures in different wavelengths, etc. 
In the following Chapter (2), a brief introduction to the Sun and the solar corona will be given. Physical conditions, such as the optical thickness, temperature and density structure of the corona will be discussed. The electromagnetic spectrum of the corona and the processes responsible for the continuum and line emission and absorption will be discussed in the Section 2.2. The theoretical overview of the line emission spectroscopy, which is important for the correct interpretation of the imaging and spectroscopic images will be given in the Section 2.2.3. The method used for computation of the Doppler shifts will be outlined in the Section 2.2.3.2.

The theory of magnetohydrodynamics (MHD), which is a plausible approximation for the theoretical interpretation of the eruptive processes and coronal waves and oscillations will be presented in the Chapter 3. The parameters characterizing the physical conditions found in the corona will be defined in the Section 3.2. Their relationship to the topology of the magnetic fields will be illustrated in the Sections 3.3, 3.3.1 and 3.4. The observational and theoretical knowledge on solar flares, filament eruptions and CMEs, relevant to the presented work can be found in the Chapter 4 . The physical conditions leading to the eruptions and a brief theoretical overview of the process of magnetic reconnection which plays an important role in the flare-CME models (Section 4.4) will be presented in the Section 4.1. The emission mechanisms related to flares, which are helpful in identification of different stages of the evolution, will be presented in the Section 4.2.1.

The theory and observations of coronal waves and oscillations will be discussed in the Chapter 5. The typical phase speeds of the magnetohydrodynamic waves helpful in identification of the observed modes in the Chapter 6 will be defined in the Section 5.2.1. In the section 5.2.2, the derivation of the dispersion relation and its solutions in a homogeneous unbounded medium can be found. In the Section 5.2.3, the dispersion relation for MHD surface and body modes in a magnetic cylinder, and a description of the wave modes which arise in the corona and are relevant to the studies in the Chapter 6 will be presented.

We have used wavelet based techniques for the enhancement of the contrast of the coronal structures in TRACE images, and to pick out the oscillations and estimate their periods in SUMER time series. They will be explained in the Section 6.2. The specifics of the instruments used for the observations will be presented in the Section 6.1 and the detailed studies of multi-wavelength observations of waves and oscillations associated with a flare, a filament eruption and a CME will be presented and summarized in the Sections 6.3 and 6.4 and 6.5 . Future possibilities for similar studies will be mentioned in the Chapter 7 


\section{The Sun and the Solar Corona}

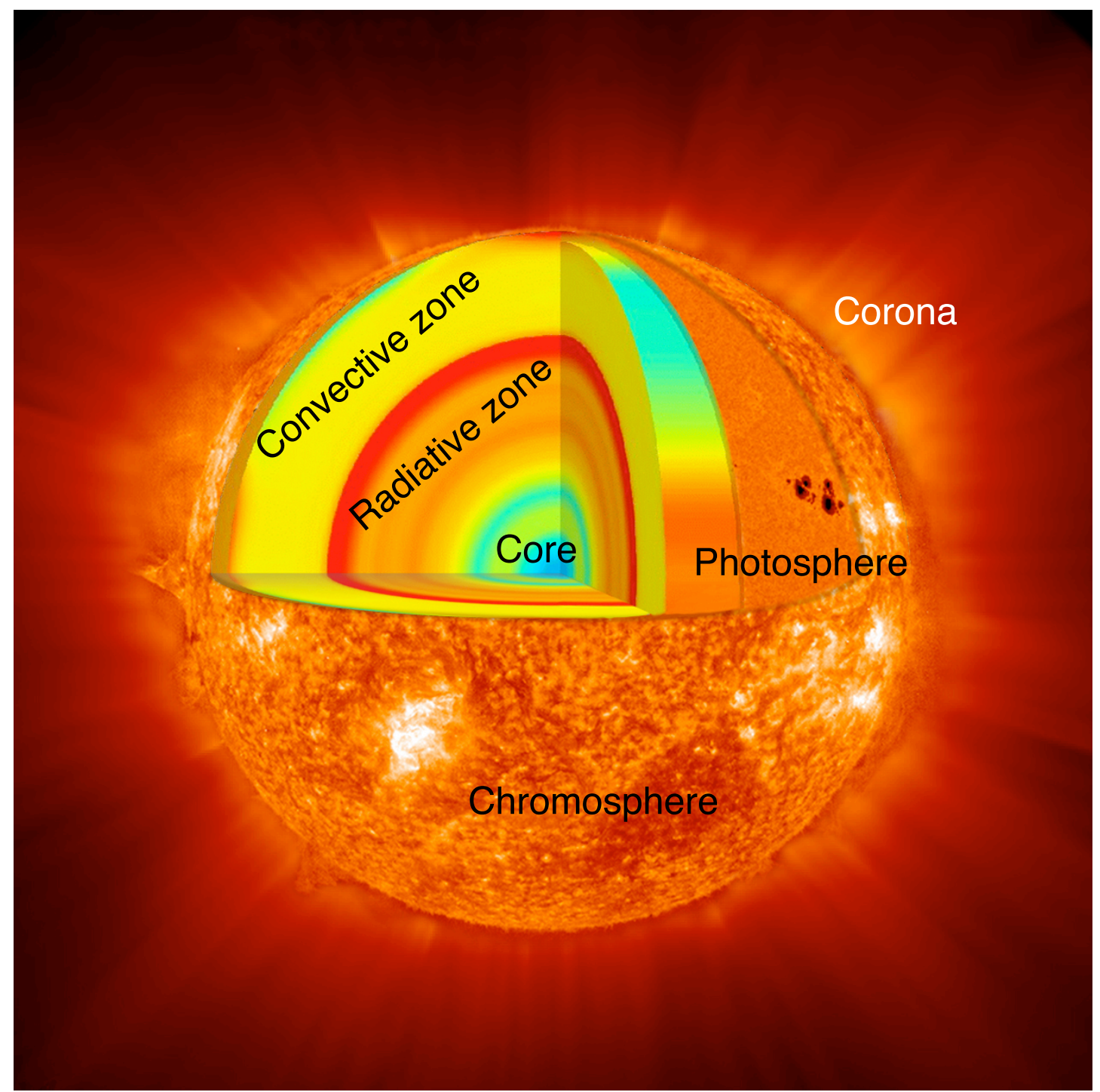

Figure 2.1: Layers of the Interior of the Sun and its atmosphere. Courtesy of NASA. 12 


\subsection{The Sun and its Atmosphere}

The solar interior shown in Fig. 2.1 consists of the core, where the energy is generated via fusion, a radiative zone through which the energy is transported via radiative transfer (A.4), and a convective zone where the transport by convective cells occur. Although electromagnetic waves are completely absorbed by the opaque interior, its structure can be studied through the waves by means of helioseismology.

The photosphere is a thin layer above the turbulent convection zone. The convection cells on its surface form granules with a lifetime of several minutes, and supergranules, which last from a few hours to days. Their boundaries are the place where the magnetic flux is concentrated in the quiet Sun. The photosphere is the the visible surface of the Sun. It is characterized by nearly black-body radiation given by Planck's law (Eq. A.15), with an effective temperature $T_{\text {eff }} \approx 5800 \mathrm{~K}$. Its particle density is around $10^{17} \mathrm{~cm}^{-3}$.

The temperature structure of the solar atmosphere is illustrated in Fig. 2.2.

Above the photosphere is the chromosphere. It is hotter $(T>10000 \mathrm{~K})$ and rarer $\left(10^{11} \mathrm{~cm}^{-3}\right)$ than the photosphere, which can be seen in Fig. 2.2. Its faint visible light is only seen when the Sun is occulted and is mostly due to the emission in lines. The red $\mathrm{H} \alpha 6562,8 \AA$, with formation temperature $T \sim 3 \times 10^{4} \mathrm{~K}$ is the most prominent. The outer layer of the solar atmosphere is the corona, filled with hot $(T>1 \mathrm{MK})$, tenuous $\left(10^{8} \mathrm{~cm}^{-3}\right)$ plasma dominated by magnetic fields. The transition region, a thin boundary between the chromosphere and the corona, is a layer where plasma becomes fully ionized. It is characterized by emission lines predominantly formed in the far ultraviolet and soft X-ray range. The transition region separates the lower layers, which are dominated by gravity, from the magnetic corona.

Solar activity is due to magnetic fields generated via dynamo processes in the solar interior and convectively transported to the surface. In the photosphere, the solar activity manifests itself in pairs of sunspots. Sunspots are cooler and hence darker (Fig. 2.1) than the surrounding photosphere and have a strong concentration of magnetic flux. In the corona, magnetic activity is seen as magnetic loops, brightenings and flares, filament eruptions, CMEs, etc. 


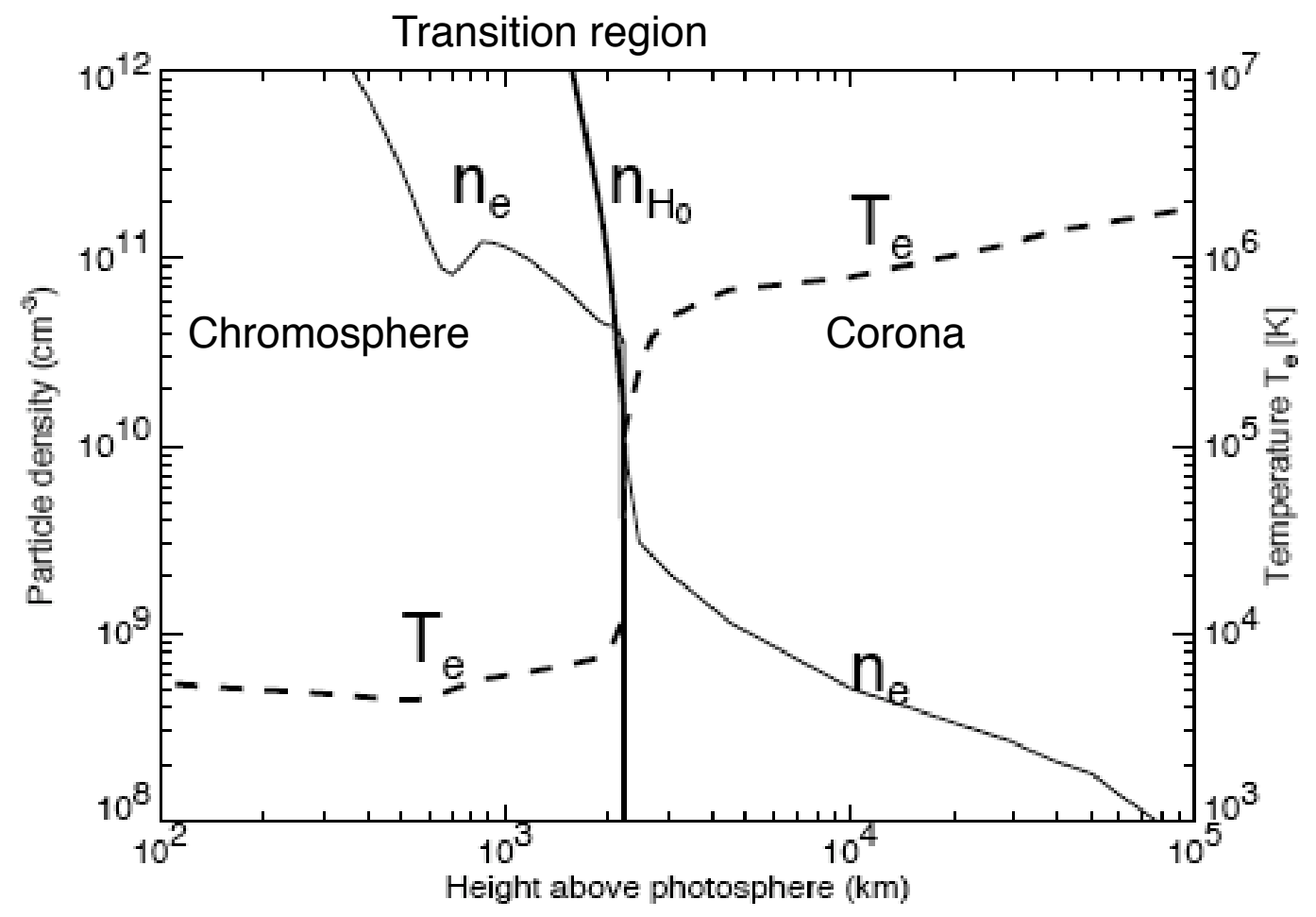

Figure 2.2: Electron density and temperature model of the chromosphere (Fontenla et al. 1990); and lower corona (Gabriel 1976).

\subsection{The Corona}

White (visible) light from the corona is 6 orders of magnitude less intense than the photospheric radiation itself. Therefore, it can only be seen when the solar surface is occulted (Fig. 2.3), either during total solar eclipses (Fig. 2.3), or using coronagraphs, discovered by Bernard Lyot at Pic-du-Midi Observatory in 1930. Contrary to the white light emission, the corona is much brighter in X-ray and radio wavelengths compared to the photosphere, which is essentially invisible.

The optical continuum emission of the solar corona, also called K-corona (from german word Kontinuum), is produced by electron Thomson scattering of the radiation emitted at the photosphere and has a shape close to the black-body radiation curve of the photosphere given by the Planck function (Eq. A.15). The scattering rate is proportional to the electron density and thus provides a method for determining the electron 


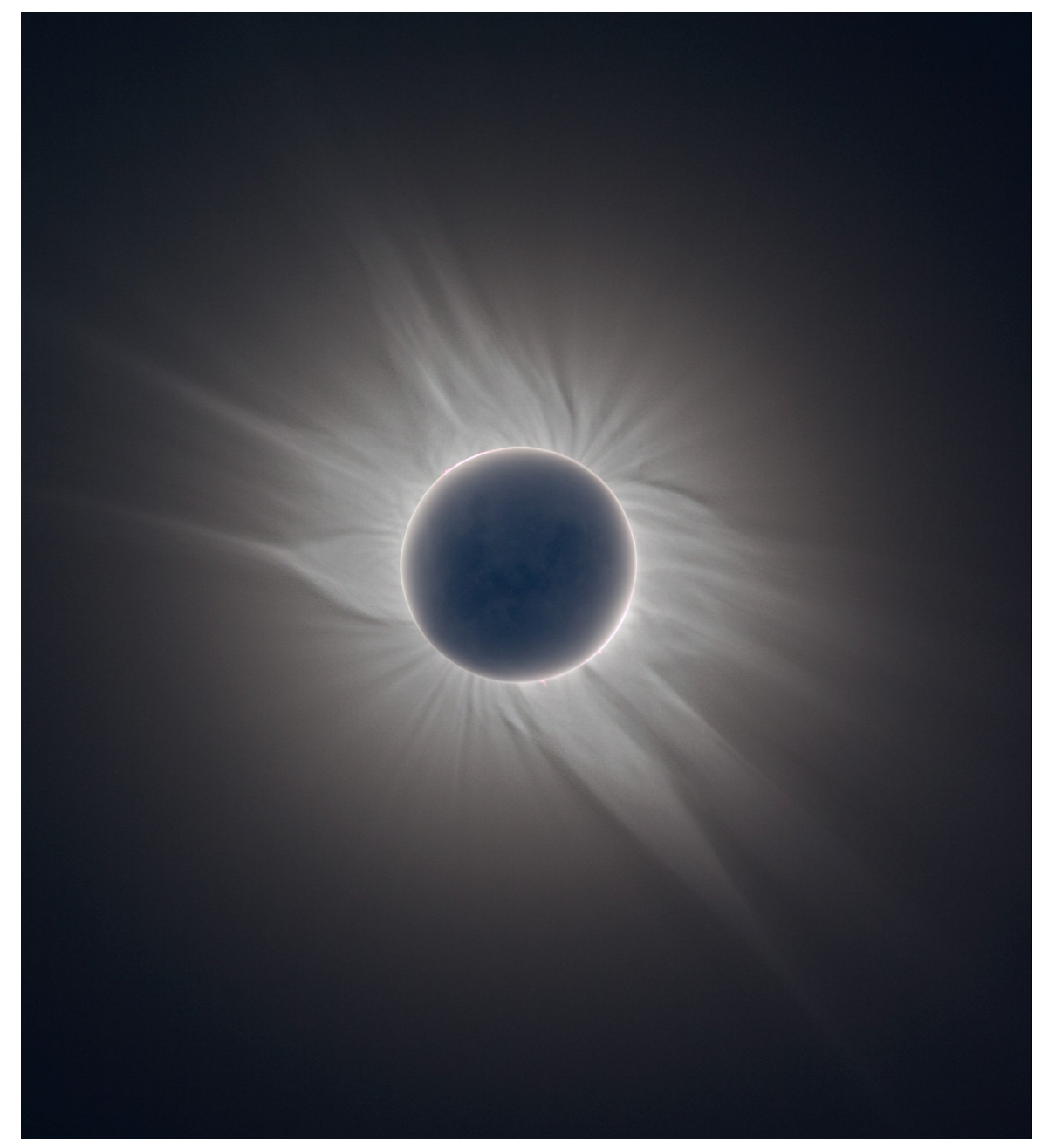

Figure 2.3: Solar eclipse, March 29, 2006.

(adopted from King (http : //spaceweather.com/eclipses/29mar06c/))

density in the solar corona.

The magnetic structure of the corona can be seen in Fig. 2.3. The large-scale loop-like structures are called helmet streamers, the open regions correspond to the coronal holes, from where the fast solar wind escapes along the magnetic field lines.

The spectrum of the Fraunhofer (F)-corona, which dominates from about 2 and 3 solar radii outwards, is formed by the scattering of the photospheric white and UV light by slowly moving interplanetary dust particles in the close vicinity of the Sun. Therefore, it has dark absorption lines (Fig. 2.4) superimposed on the continuum. The Fraunhofer $\mathrm{H} \alpha$ line at $6563 \AA$ discussed in 6.3 and 6.4 belongs to the Balmer series of 
Hydrogen.

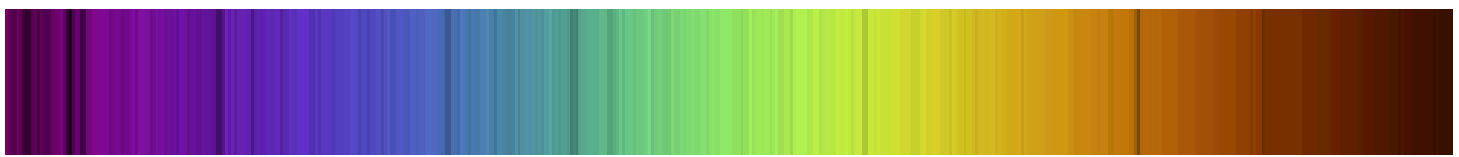

Figure 2.4: Fraunhofer absorption lines in the solar visible spectrum ranging $4000<$ $\lambda<7000 \AA$. (adopted from http://bass2000.obspm.fr)

Fraunhofer lines are formed mainly via absorption of a photon. The transition rate is proportional to the energy density of the radiation field, the number of atoms in the lower energy state and Einstein's coefficient for induced absorption.

The Emission (E)-corona is the component of the coronal radiation characterized by emission continuum (Fig. 2.7) and emission lines (Fig. 2.8). A composite image taken by TRACE (Fig. 2.5) represent observations in EUV lines Fe Ix /Fe x $171 \AA$ (red color table), Fe xII $195 \AA$ (green), and Fe xv $284 \AA$ (blue) corresponding to temperatures 1.3 MK, 1.6 MK and 2 MK respectively. In this Section, a summary of the processes responsible for continuum and line emission in the corona will be given. More details on the signatures of flares provided by observations can be found in the Section 4.2 .1 .

$\mathrm{b}$

\subsubsection{Emission Measure and Differential Emission Measure}

The EUV emission in the solar corona is optically thin. The observed EUV radiation is due to the integrated emission from transitions in all ions along the line-of-sight. Therefore, coronal continuum and line intensity are often expressed in terms of (differential) emission measure $(D) E M$, a quantity characterizing the temperature structure of the EUV corona. Emission measure is defined as:

where $z$ is the distance along the of the emitting plasma. Examples of $D E M$ distributions are shown in Fig. 2.6. The DEM distribution of the flaring plasma (red curve in Fig. 2.6) shows a local maximum at a temperature $T \sim 12 \mathrm{MK}$, implying that its spectrum is dominated by emission lines due to transitions of highly ionized ions formed at around this temperature. DEM maxima for active region (blue), quiet sun (black solid), and prominence (cyan) plasmas are at temperatures 1-1.5 orders lower. 


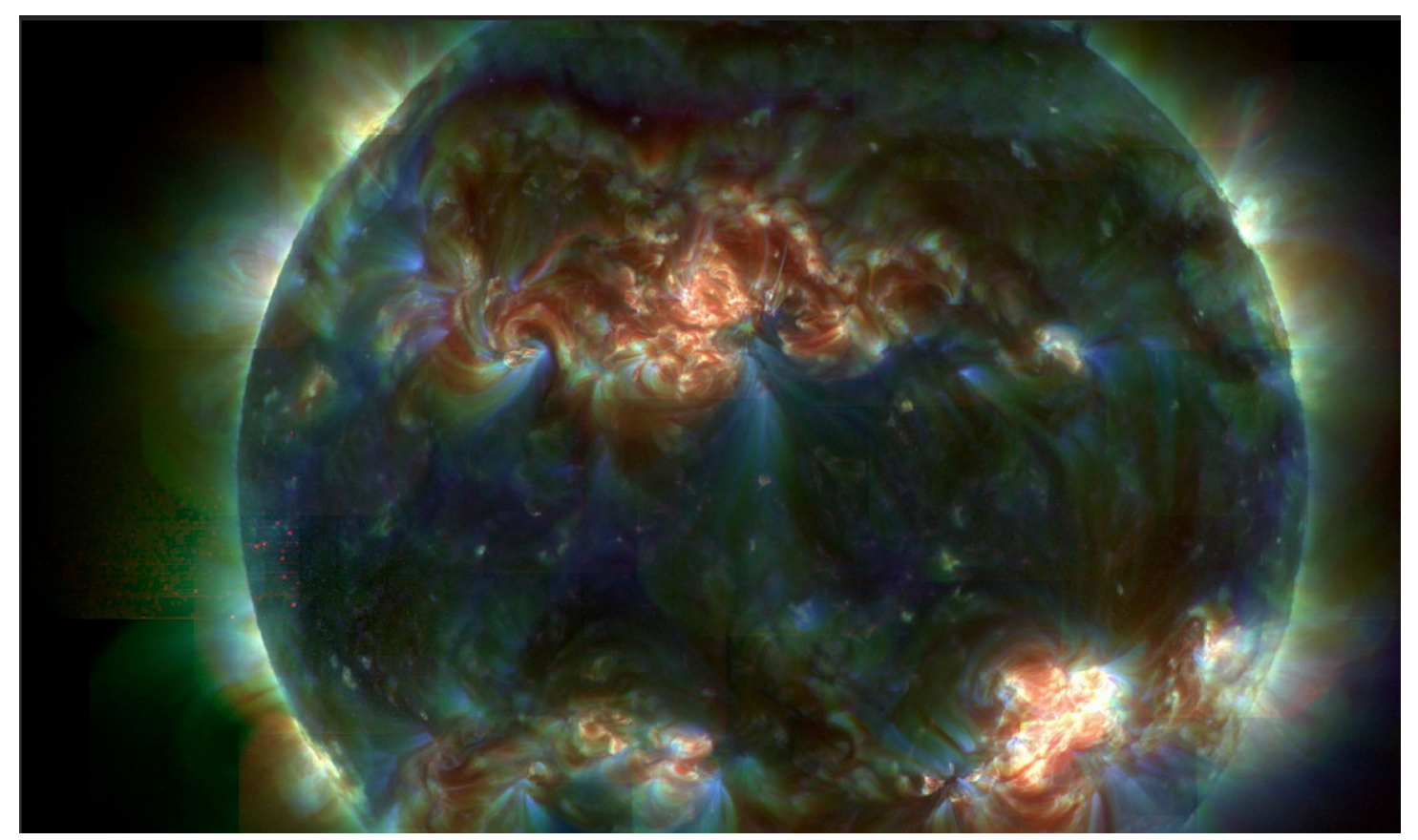

Figure 2.5: TRACE composite image.

(courtesy http : //trace.lmsal.com/POD/images/TRACE picturebook.jpg)

\subsubsection{Continuum Spectrum of the Corona}

\subsubsection{Free-free Continuum}

The most common radiative process occurring in the corona is Bremsstrahlung (German term for braking radiation), discovered by Tesla and named by Bohr. It is a free-free (i.e. not ionizing) transition due to the non-elastic scattering of free electrons. Thermal/nonthermal Bremsstrahlung radiation is emitted when Maxwellian/non-Maxwellian electrons, respectively, are decelerated in the Coulomb field of ions.

\section{Thermal Bremsstrahlung Emission}

Thermal Bremsstrahlung on highly ionized coronal ions produces soft X-ray (SXR) photons with wavelengths in the range $(1<\lambda<100 \AA)$. The effective temperature of SXR radiation ranges between 1,5-150 MK. EUV continuum (100-1000 $\AA$ ) is produced in the same process, though the degree of ionization of the collisional ions is much lower in this case and so is the effective electron temperature ( $\leq 1,5 \mathrm{MK})$. For Hydrogen-like atoms, at a temperature $T_{e}$, the power emitted per unit volume and unit wavelength interval due to Bremsstrahlung from ions with charge $|Z e|$ is (Tucker \& Gould 1966) 


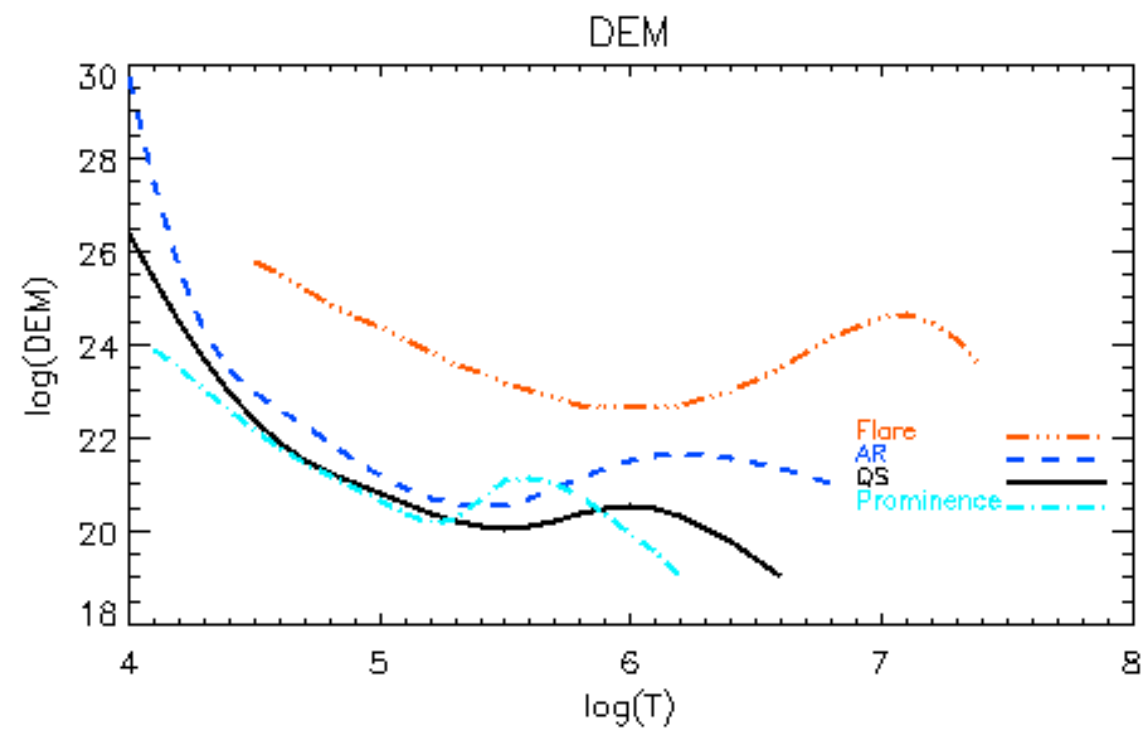

Figure 2.6: Distribution of $D E M$ of the coronal plasma under various conditions found in the corona obtained using CHIANTI database.

$$
\frac{d P_{\lambda}^{f f}}{d V d \lambda} \approx 2.04 \times 10^{-19} \lambda^{-2} T^{-1 / 2} \exp \left(-\frac{h c}{\lambda T}\right) N_{e} N\left(X^{+Z}\right) g_{\mathrm{ff}} \quad\left[\operatorname{erg~cm}^{-3} \mathrm{~s}^{-1} \AA^{-1}\right],
$$

where $g_{\mathrm{ff}}(T, \lambda)$ is a velocity averaged Gaunt factor, which is of the order of unity under coronal conditions (Karzas \& Latter 1961). Bremsstrahlung is characterized by a continuous distribution of radiation (black curve in Fig. 2.7), which becomes more intense and shifts toward higher frequencies when the energy of the bombarding electrons is increased.

\section{Non-thermal Bremsstrahlung Radiation}

During the most energetic processes in large flares, the relativistic electrons accelerated in the collisionless corona precipitate to the dense chromosphere, where they interact with atomic nuclei, and $\gamma$-rays are emitted. $\gamma$-photons are characterized by the shortest wavelength $\left(10^{-3}-10^{-1} \AA\right)$ in the solar spectrum, and by corresponding energies (100 $\mathrm{keV}-10 \mathrm{MeV})$.

When mildly non-thermal relativistic electrons $(0,2 c-0,5 c)$ decelerate in collisions with the chromospheric ions in a process called thick-target (non-thermal) Bremsstrahlung, hard X-ray (HXR) wavelengths $\left(10^{-1}-1 \AA\right.$ with corresponding energies of $\left.10-100 \mathrm{keV}\right)$ are observed . 


\subsubsection{Free-bound Continuum}

The free-bound continuum emission (red curve in Fig. 2.7) is due to radiative recombination of ions in collisions with electrons. It involves a capture of a free electron to a bound state $n$, hence the name free-bound. An inverse process to radiative recombination is the photo-ionization. The sharp edges which can be seen in Fig. 2.7 are due to the recombination at wavelengths $\lambda=h c / E_{N}^{Z}$ corresponding to ionization energies $E_{n}^{Z}$ of different levels $n$.

The rate of photo-ionization is proportional to the energy density of the radiation field, which is weak in the corona. It is therefore often omitted in the ionization equilibrium calculations in favor of the collisional ionization, which is dominant. The energy of the photon emitted by a stationary ion in charge $Z$ and level $n$ due to the capture of an electron with the speed $\mathrm{v}$ is:

$$
h v=E_{n}^{Z}+\frac{1}{2} m_{e} \mathrm{v}^{2} .
$$

It follows from (Eq. 2.3), that the free-bound emission and absorption have a the maximum wavelength cut-off at $\lambda_{\max }^{Z}=h c / E_{n}^{Z}$, which is $912.13 \AA$ for the ground state of the Hydrogen atom. Tucker \& Gould (1966) calculated the the radiative recombination emission spectrum for Hydrogen-like atoms. The total power emitted via radiative recombinations of ion $X$ into the excited state $n$ and ionization degree $|Z|$ is

$$
\begin{aligned}
& \frac{d P_{\lambda}^{f b}}{d V d \lambda} \approx 6.52 \times 10^{-14} \lambda^{-2} T^{-3 / 2} \exp \left(-\frac{h c}{\lambda k_{B} T_{e}}\right) N_{e} N\left(X^{+Z+1}\right) . \\
& \zeta_{n} n\left(E_{n}^{Z} / E_{\mathrm{H}}\right)^{2} \exp \left(E_{\mathrm{H}} / k_{B} T\right) g_{\mathrm{fb}} \quad\left[\mathrm{erg} \mathrm{cm}^{-3} \mathrm{~s}^{-1} \AA^{-1}\right] \\
& \text { for } \lambda<\lambda_{\max }^{Z}=\frac{h c}{E_{n}^{Z}} \approx \frac{12400}{E_{n}^{Z}} \quad[\AA] .
\end{aligned}
$$

where $\zeta_{n}$ is the incomplete fraction of the shell $n$.

\subsubsection{Two-photon continuum}

The green curve in Fig. 2.7 represents the continuum emission due to two-photon emission. Simultaneus emission of two photons with wavelengths $\lambda_{1}=c / \nu_{1}$ and $\lambda_{2}=c / v_{2}$ results from a radiative de-excitation from the metastable states $2 S(n=2, J=0)$ characterized by energy $E_{u}$, to the ground state $1 S(n=1, J=0)$ with energy $E_{l}$.

$$
h v_{1}+h v_{2}=E_{\mathrm{ul}}=E_{u}-E_{l}
$$

In Hydrogen- and Helium-like ions, the rate of excitation to the metastable levels $2 S$ is about one third of the excitation rate to the level $2 P$ (Beigman et al. 1970). Therefore, if a one-photon depopulating process is strictly forbidden by the quantum rules, 
and the rate of collisional de-excitation is small, the energy of two-photon emission will be one third of the energy emitted in neighbouring $2 P-1 S$ transitions. According to Spitzer \& Greenstein (1951), the rate of two-photon emission for hydrogenic ions is $8.23 \mathcal{Z}^{6} \mathrm{~s}^{-1}$, while the corresponding probability of single-photon emission is negligible. Spitzer \& Greenstein (1951) have calculated the shape of the two-photon continuum for Hydrogen. Its frequency ranges from $0<v_{\mathrm{tp}}<E(2 S) / h$ and is symmetric about its central frequency $1 / 2 E(2 S) / h$, which is also the most probable one. Assuming that all Hydrogen-like ions have a similar spectral shape, Tucker \& Koren (1971) evaluated the energy per unit volume, time, and wavelength emitted due to two-photon de-excitation in a hydrogenic ion with a single electron:

$$
\frac{d P_{\mathrm{tp}}(Z)}{d \lambda d t}=\frac{4 P_{\mathrm{L} \alpha}(T)}{\lambda}\left(\frac{\lambda_{\mathrm{ul}}}{\lambda}\right)^{3}\left(1-\frac{\lambda_{\mathrm{ul}}}{\lambda}\right) \quad\left[\mathrm{erg} \mathrm{cm}^{-3} \mathrm{~s}^{-1} \AA^{-1}\right],
$$

where $\lambda_{\mathrm{ul}}=\frac{\lambda_{1} \lambda_{2}}{\lambda_{1}+\lambda_{2}}$ is the wavelength corresponding to the energy difference $E_{\mathrm{ul}} . P_{\mathrm{L} \alpha}$ is the power of the neighbouring $2 P-1 S$ single-photon emission, which corresponds to $\lambda_{\mathrm{L} \alpha}=1215.7 \AA$ for $\mathrm{H}_{\mathrm{I}}$ and $303.8 \AA$ for He II .

In Helium-like atoms, the two-photon process is unimportant from the state $2^{3} S$ Griem (1969) because a single-photon magnetic dipole de-excitation is allowed. According to Tucker \& Koren (1971) the power of the two-photon transition of an ion in a charge state $(Z-1)$ can be calculated when $P_{\mathrm{L} \alpha}(T)$ in Eq. 2.6 is replaced by the sum of the powers of the transitions i $2^{3} P-1{ }^{1} S, 2^{1} P-1{ }^{1} S$ and $2^{3} S-1{ }^{1} S$ of the ion in the charge state $(Z-2)$.

\subsubsection{Synthetic CHIANTI Continuum Spectrum}

Fig. 2.7 represents the continuum emission spectrum calculated using the CHIANTI database according to the model by Gronenschild \& Mewe (1978). It assumes stationary, optically thin plasma in ionization equilibrium with a Maxwellian electron velocity distribution A.6. The mentioned assumptions may be violated during flares when the plasma temperature changes are faster than ionization changes leading to nonequilibrium ionization, and electrons are accelerated to form non-Maxwellian distributions. Blue, black, red and green curves in Fig. 2.7 correspond respectively the total, free-free, free-bound and two-photon continuum energy emitted per unit time, angle, emission measure and wavelength. The coronal abundances used in calculation were adopted from Feldman et al. (1992) and the ion population is given by the ionization balance of Mazzotta et al. (1998). In the spectroscopic observations in the Chapter 6 , the continuum emission has been subtracted from the spectrum in order to represent the line emission and to pick out the Doppler shifts. 


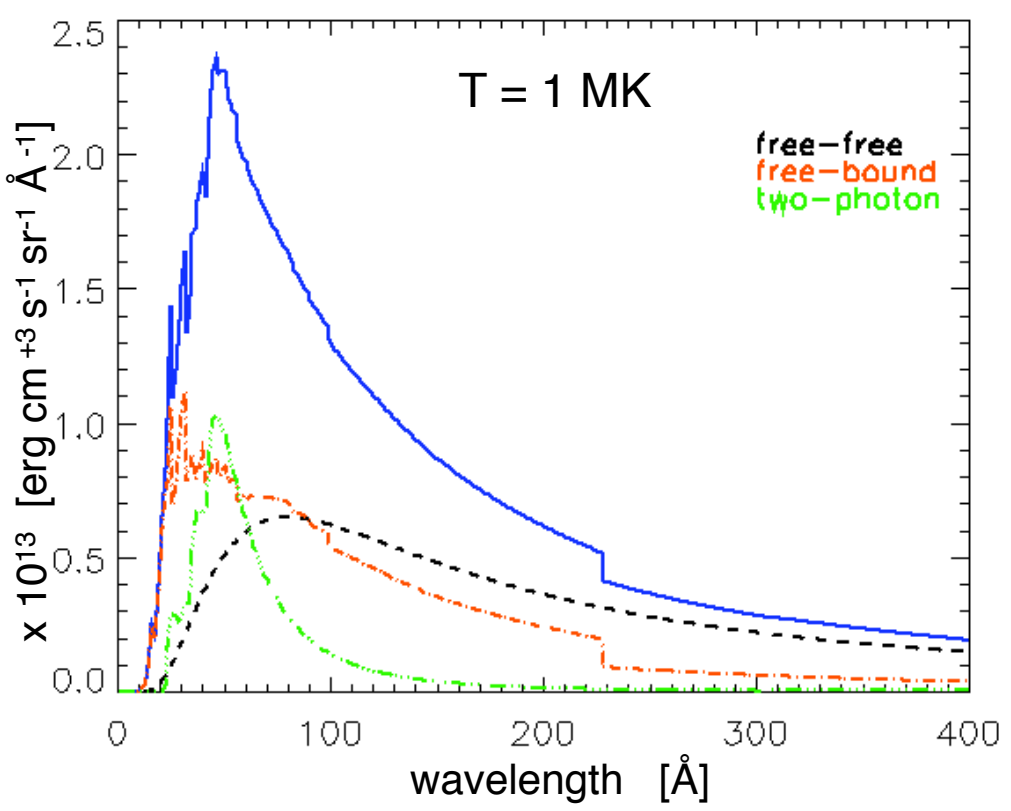

Figure 2.7: Continuum emission calculated using CHIANTI atomic database.

\subsubsection{Emission Lines}

The EUV and soft X-ray spectrum of the corona is dominated by emission lines superimposed on the emission continuum. The optical part of the corona contains a number of emission lines, among which the most prominent ones are the forbidden green Fe xIV 5302,8 $\AA$ and red line Fe x $6374 \AA$ A. Edlen and Grotarian (1939) showed, that the green coronal line, originally associated to the unknown element "coronium", is formed in a highly improbable transition forbidden by quantum-mechanical rules. The likeliness of occurrence of "forbidden" transitions becomes much higher in the hot and tenuous plasma of the corona, where the collisional de-excitation from meta-stable states is rare in comparison with the radiative decay.

\subsubsection{EUV Emission Line Spectroscopy}

The dominant processes in optically thin coronal plasma are collisional ionization balanced by radiative recombination, and collisional excitation balanced by radiative deexcitation (spontaneous decay), which is much more significant than the de-excitation by collisions, owing to the long mean free path of the free electrons. Absorption and stimulated emission, both proportional to the radiation field are negligible, therefore, there is no need to solve the radiative transport equation A.3. 
The specific intensity (more correctly specific or spectral radiance) $I\left(\lambda_{\mathrm{lu}}\right)=I_{\mathrm{lu}}$ of an optically thin spectral line of wavelength $\lambda_{\text {lu }}$ is the energy emitted per unit time, unit area, unit angle and wavelength interval:

$$
I\left(\lambda_{\mathrm{lu}}\right) d \lambda_{\mathrm{lu}}=\frac{h v_{\mathrm{lu}}}{4 \pi} \int N_{u}\left(X^{+Z}\right) A_{\mathrm{ul}} d z \quad\left[\operatorname{erg~cm}^{-2} \mathrm{~s}^{-1} \mathrm{ster}^{-1}\right]
$$

The population density $N_{j}\left(X^{+Z}\right)$ can be expressed as a product of five terms:

- excitation ratio - the relative upper level (j) population $N_{j}\left(X^{+Z}\right) / N\left(X^{+Z}\right)$

- ionization ratio - the relative abundance of the ion $N\left(X^{+Z}\right) / N(X)$

- element abundance relative to the abundance of Hydrogen $N(X) / N_{\mathrm{H}}$

- ratio of the Hydrogen to the electron density $N_{\mathrm{H}} / N_{e}$

- electron density $N_{e}$

It follows, that

$$
N_{j}\left(X^{+Z}\right)=G\left(T, \lambda_{\mathrm{lu}}\right) N_{e} N_{\mathrm{H}} A_{\mathrm{ul}}^{-1},
$$

where

$$
G_{\mathrm{lu}}(T)=G\left(T, \lambda_{\mathrm{lu}}\right)=\frac{h c}{4 \pi \lambda_{\mathrm{lu}}} \frac{N_{j}\left(X^{+Z}\right)}{N\left(X^{+Z}\right)} \frac{N\left(X^{+Z}\right)}{N(X)} \frac{N(X)}{N_{\mathrm{H}}} \frac{A_{\mathrm{ul}}}{N_{e}} \quad\left[\mathrm{erg} \mathrm{cm}^{+3} \mathrm{~s}^{-1} \mathrm{ster}^{-1}\right]
$$

and 2.7 can be re-written in terms of the Contribution function $G\left(T, \lambda_{\mathrm{lu}}\right) 2.9$ ) of the line:

$$
I_{\mathrm{lu}} d \lambda_{\mathrm{lu}}=\int G_{\mathrm{lu}}\left(T, \lambda_{\mathrm{lu}}, N_{e}\right) N_{e} N_{\mathrm{H}} d z \quad\left[\mathrm{erg} \mathrm{cm}^{-2} \mathrm{~s}^{-1} \mathrm{ster}^{-1}\right],
$$

This form of the equation 2.10 is convenient under the coronal approximation, in which excitation rates are much faster that the ionization and recombination processes, and the ionization and excitation equilibria can be calculated separately, giving the relative upper level population and the ion abundance. If the electron density $N_{e}$ and temperature $T_{e}$ don't vary along the line of sight, 2.11 can be expressed in terms of the emission measure defined above (2.1):

$$
I\left(\lambda_{\mathrm{lu}}\right)=G_{\mathrm{lu}}\left(T, N_{e}\right)<E M>\quad\left[\mathrm{erg} \mathrm{cm}^{-2} \mathrm{~s}^{-1}\right]
$$




\subsubsection{Line Characteristics}

Computation of the spectral characteristics of the observed lines allow us to determine the total radiance $I$ emitted in a wavelength interval, Doppler shift $\Delta \lambda$ of the center of the line and the line width $w$ :

- Integrated radiance (intensity) $I$, as as the zeroth moment of the specific intensity $I_{\lambda}$ :

$$
I=M_{0}=\int I_{\lambda} d \lambda \quad\left[\mathrm{erg} \mathrm{cm}^{-2} \mathrm{~s}^{-1}\right]
$$

- Doppler shift $\Delta \lambda$ of the center of gravity of the line is the ratio of the first and zeroth weighted moments:

$$
\Delta \lambda=M_{1} / M_{0}=\frac{\int I_{\lambda} \lambda d \lambda}{\int I_{\lambda} d \lambda} \quad[\AA],
$$

representing the velocity of the majority of the plasma with the temperature around the formation temperature of the given line. Bulk Doppler line-of-sight velocity $\mathrm{v}_{\mathrm{d}}$ of the plasma emitting at a shifted wavelength $\lambda \pm \Delta \lambda$ is:

$$
\mathrm{v}_{\mathrm{d}}=\mathrm{c} \cdot \frac{\Delta \lambda}{\lambda} \quad\left[\mathrm{ms}^{-1}\right]
$$

- Line width $w$ of a spectral line is defined as the difference of the squares of the second and first weighted moment,

$$
w=\sqrt{\left(M_{2} / M_{0}\right)^{2}-\left(M_{1} / M_{0}\right)^{2}}=\sqrt{\left[\frac{\int I_{\lambda} \lambda^{2} d \lambda}{\int I_{\lambda} d \lambda}\right]^{2}-\Delta \lambda^{2}}[\AA] .
$$

Full width at half maximum is another measure of line broadening, defined as a difference of the extreme values of the wavelengths of the Gaussian 2.17 fitted to the observed intensity, measured at the half of its maximum value.

$$
F W H M=2 \sqrt{2 \ln 2} \sigma \quad[\AA],
$$

where $\sigma$ is a standard deviation of the Gaussian distribution

$$
f(\lambda)=\frac{1}{\sigma \sqrt{2 \pi}} e^{-\frac{\left(\lambda-\lambda_{0}\right)^{2}}{2 \sigma^{2}}} .
$$




\subsubsection{Synthetic CHIANTI Emission Line Spectrum}

The synthetic EUV spectra in Fig. 2.8 were calculated using the CHIANTI database under isothermal assumption for temperatures $T=1 \mathrm{MK}$ (blue), $T=10 \mathrm{MK}$ (black), $T=100 \mathrm{MK}$ (red) and a density $N_{e}=10^{8} \mathrm{~cm}^{-3}$. For the reasons of better representation, maximum intensities at each temperature were normalized. The wavelength range covers the lines observed with the TRACE imager. Their position is marked. At flare temperatures $(T \sim 100 \mathrm{MK})$, the most prominent line is Fe xxIv $192 \AA$ A.3.2. It is covered by the $195 \AA$ ATRACE band which, at lower temperatures is dominated by the Fe XII emission. The coronal abundances and ion populations were adopted from Feldman et al. (1992) and Bryans et al. (2009), respectively.

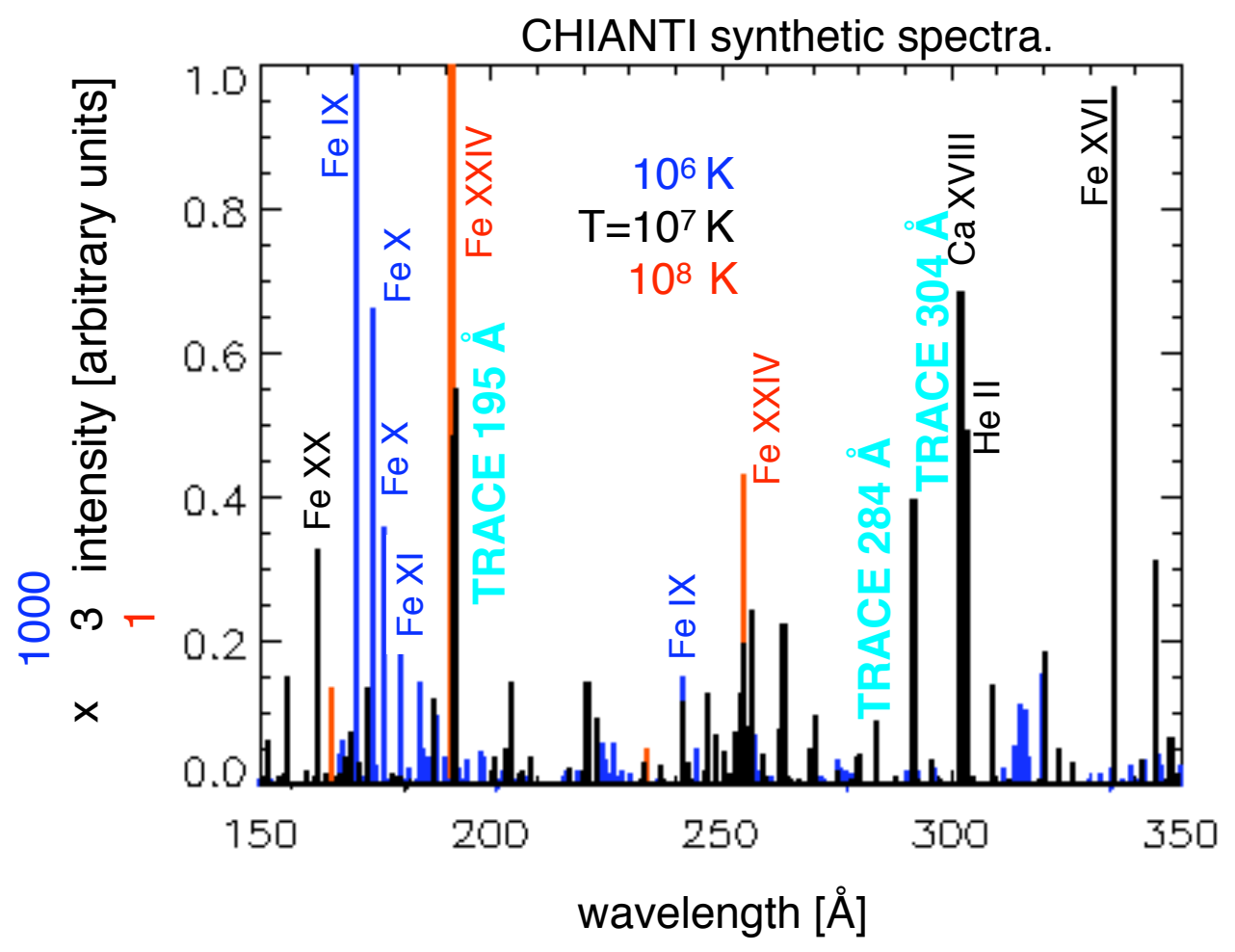

Figure 2.8: EUV emission lines in the wavelength range $150 \AA<\lambda<350 \AA$ calculated using CHIANTI atomic database. The TRACE EUV lines are marked. 


\subsubsection{SUMER EUV Spectrum}

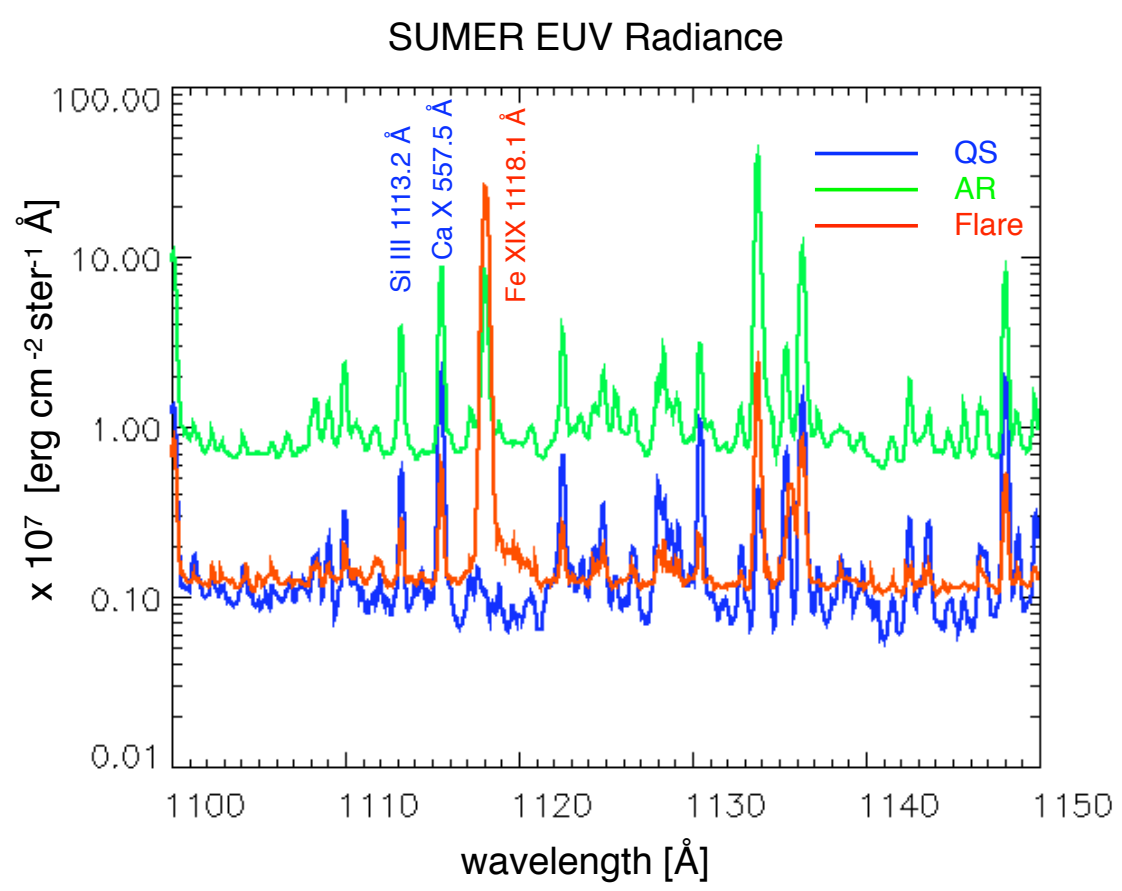

Figure 2.9: SUMER radiance as observed on quiet Sun (blue), active region (green) and flaring plasma (red) (Curdt et al. 2004).

The SUMER spectrometer observes in the far ultraviolet range in two orders, so that the first and second order lines are superimposed. It covers the EUV wavelengths from 500-1610 А. The spectrum range discussed in the Chapter 6 is illustrated in Fig. 2.9. The second order range 1100/2 $\mathrm{A}<\lambda<1150 / 2 \AA$ is superimposed on the first order range $1100 \AA<\lambda<1150 \AA$. A line list of the EUV emission lines and their blends relevant to our studies can be found here: (A.3). The SUMER lines discussed in the Sections 6.3 and (6.4) are marked.

The $\mathrm{Si}$ III line at $1113,2 \AA$ with the formation temperature $\sim 10^{4} \mathrm{~K}$ results from the dipole allowed $(\Delta J=0, \pm 1, \Delta s=0) 3 s 3 p^{3} P_{2}-3 s 3 d^{3} D_{1,2,3}$ transitions. The $\mathrm{Ca} x$ line at $557.8 \AA$ resulting from a dipole allowed transition ${ }^{3} s^{2} S_{1 / 2}-{ }^{3} p^{2} P_{3 / 2}$ is formed at coronal temperatures ( 0.6 MK). The Fe XIx line $1 s^{2} 2 s^{2} 2 p^{43} P_{2}-{ }^{3} P_{1}$ at $1118.06 \AA$ ( $\left.T \sim 10 \mathrm{MK}\right)$ results from an intercombination (spin-forbidden or semi-forbidden) magnetic dipole $(\Delta s \neq 0, \Delta J=0, \pm 1)$ transition within the ground configuration and was identified by (Doschek et al. 1975). High shifts (up to $650 \mathrm{~km} \mathrm{~s}^{-1}$ ) and a large Doppler broadening as well as Doppler shift and Doppler width oscillations have been observed in this line. 
Hence, it plays a very important role as a UV diagnostic of the dynamics before, during and after a flare.

The intensity of $\mathrm{Ca}$ x line which is observed in the second order, cannot be quantitatively compared to the first order lines, because radiometric calibration for first order lines was performed. The observed intensity of the continuum and of the different lines depends upon the temperature and density structure of the region where they are formed. 


\section{Magnetohydrodynamics}

The solar magnetic field is very complicated and its study requires some basic assumptions and simplifications. Hydrostatics (HS) is a tool allowing us to study the coronal plasma in terms of simple fluid mechanics with no magnetic fields and flows. In order to include the flows, hydrodynamics (HD) needs to be applied, which, combined with the theory of electromagnetism gives magnetohydrodynamics, a plausible approximation for the study of the various phenomena taking place in the coronal plasma. The coronal magnetic field plays an important role in channeling charged particles and ions, plasma and heat flows, waves, etc. It may change the topology by exerting a Lorentz force on the plasma, building up and storing the magnetic energy, triggering instabilities, reconnecting, etc.

\subsection{MHD Equations}

MHD equations include three conservation laws together with Maxwell's differential equations $3.103 .11,3.12,3.13$ describing the relation between the electric field $\mathbf{E}$, magnetic flux density $\mathbf{B}$ and current density $\mathbf{j}$ in the non-relativistic approximation $\mathrm{v}<<\mathrm{c}$.

Equation of continuity yields from the law of mass conservation :

$$
\frac{\partial \rho}{\partial t}+\nabla \cdot(\rho \mathbf{v})=0
$$

Equation of motion yields from the conservation of momentum of force :

$$
\frac{\partial(\rho \mathbf{v})}{\partial t}+\nabla \cdot(\rho \mathbf{v v})=-\nabla p_{\text {tot }}+\nabla \cdot \frac{\mathbf{B B}}{4 \pi}-\rho \mathbf{g}+\rho v_{\mathrm{visc}} \nabla^{2} \mathbf{v},
$$

where the total pressure $p_{\text {tot }}$ is a sum of the gas pressure, which under assumption of ideal gas is

$$
p_{g}=N k_{B} T \approx 2 N_{e} k_{B} T
$$


and magnetic pressure

$$
p_{m}=B^{2} / 8 \pi \text {. }
$$

$\mathbf{v v}($ resp.BB $)$ is a dyadic product, given by a $(3 \times 3)$ matrix:

$$
\mathbf{v} \mathbf{v}=\left(\begin{array}{c}
\mathrm{v}_{\mathrm{x}} \\
\mathrm{v}_{\mathrm{y}} \\
\mathrm{v}_{\mathrm{z}}
\end{array}\right)\left(\begin{array}{lll}
\mathrm{v}_{\mathrm{x}} & \mathrm{v}_{\mathrm{y}} & \mathrm{v}_{\mathrm{z}}
\end{array}\right) .
$$

\section{Equation of energy conservation:}

$$
\frac{\partial e_{\mathrm{tot}}}{\partial t}+\nabla \cdot\left(e_{\mathrm{tot}} \mathbf{v}+p_{\mathrm{tot}} \mathbf{v}-(\mathbf{B B}) \mathbf{v}\right)=\nabla \cdot \frac{\mathbf{B} \times \eta \mathbf{j}}{4 \pi}-Q_{\mathrm{visc}}-Q_{\mathrm{rad}}-Q_{c} .
$$

where the total energy $e_{\text {tot }}$ is a sum of kinetic, internal and magnetic energy,

$$
e_{\mathrm{tot}}=\frac{1}{2} \rho \mathrm{v}^{2}+\rho \epsilon+\frac{\mathrm{B}^{2}}{8 \pi}
$$

and $\epsilon$ is the specific internal energy, which is given by an equation of state. For an ideal adiabatic gas with an adiabatic constant $\gamma$ is

$$
\epsilon=\frac{p}{\gamma-1}=\frac{k_{B} T}{\gamma-1}
$$

The first term on the right hand side of the Eq. 3.6 represents the Ohmic losses, $Q_{\text {visc }}$ are the losses by viscous dissipation, $Q_{\text {rad }}$ are the radiative losses and $Q_{c}=\nabla \cdot \mathcal{F}_{c}=$ $-\nabla \cdot(\kappa \nabla T)$ are the conductive heat losses.

Gauss' law:

$$
\boldsymbol{\nabla} \cdot \mathbf{E}=4 \pi \sigma_{Q},
$$

Gauss' law for magnetism:

$$
\boldsymbol{\nabla} \cdot \mathbf{B}=0,
$$

Ampère's circuital law:

$$
\nabla \times \mathbf{B}=\frac{4 \pi}{c} \mathbf{j}+\frac{1}{c} \frac{\partial \mathbf{E}}{\partial t},
$$




\section{Ohm's law:}

$$
\sigma(\mathbf{v} \times \mathbf{B}+\mathbf{E})=\mathbf{j}
$$

Faraday's induction equation:

$$
\frac{\partial \mathbf{B}}{\partial t}=\nabla \times(\mathbf{v} \times \mathbf{B})+\eta \nabla^{2} \mathbf{B}
$$

or, in a conservative form:

$$
\begin{gathered}
\frac{\partial \mathbf{B}}{\partial t}+\nabla \cdot(\mathbf{v B})=\nabla \cdot(\mathbf{B v})-\nabla \times(\eta \mathbf{j}) . \\
\eta=\frac{c^{2}}{4 \pi \sigma} \quad\left[\mathrm{cm}^{2} \mathrm{~s}^{-1}\right]
\end{gathered}
$$

is called magnetic diffusivity or resistivity,

$$
\sigma=N_{e} e^{2} / m_{e} v_{c} \quad\left[\mathrm{~s}^{-1}\right]
$$

is the conductivity,

$$
v_{c} \approx 2,91 \cdot 10^{-6} N_{e} \ln \Lambda T_{e}^{-3 / 2} \quad \mathrm{~s}^{-1}
$$

is the frequency of the collisions, and $\ln \Lambda$ is the Coulomb logarithm, which measures the ratio of the efficiency of small-angle to large-angle collisions.

The first term on the right hand side of 3.13 is the convective term :

$$
\nabla \times(\mathbf{v} \times \mathbf{B})
$$

the second one is called the diffusive term:

$$
\eta \nabla^{2} \mathbf{B}
$$

\subsection{Dimensionless Plasma Parameters}

In order to simplify the MHD equations, a comparison of the effects represented by various terms in the MHD equations in the studied medium is necessary. Two important parameters describing the coronal plasma are the plasma $\beta$ parameter and the Magnetic 
Reynold's number. The latter is analogous to the viscous Reynold's number, which is not relevant in the magnetically dominated corona.

Plasma $\beta$ parameter indicates the relative importance of kinetic and magnetic processes in Eq. 3.6. It is defined as the ratio of the gas pressure $p_{g}$ (Eq. 3.3) to the magnetic pressure $p_{m}$ (Eq. 3.4):

$$
\beta=\frac{p_{g}}{p_{m}}=\frac{2 N_{e} k_{B} T}{B^{2} / 8 \pi}
$$

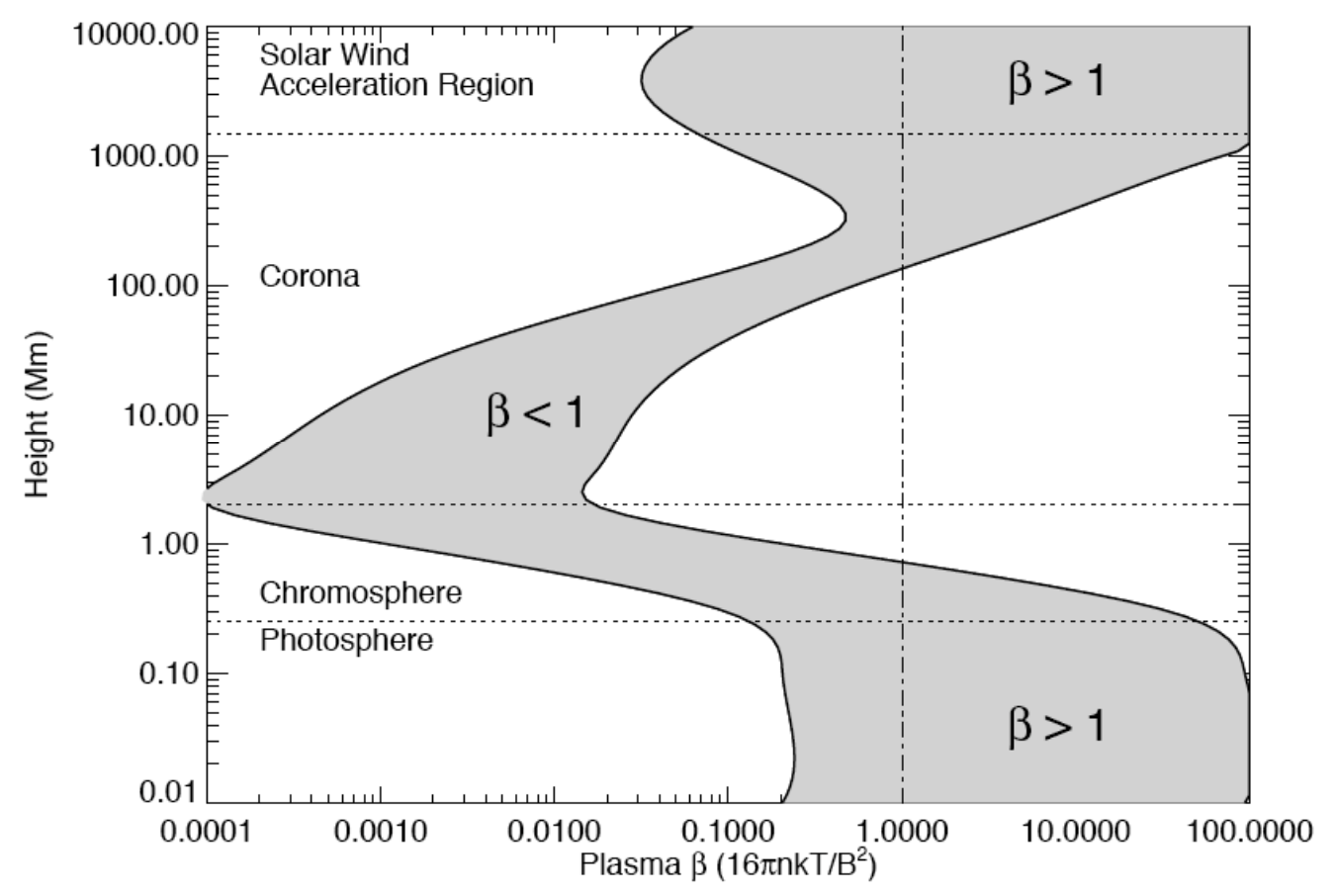

Figure 3.1: Plasma $\beta$ in the solar atmosphere for $B=100 \mathrm{G}$ and $\mathrm{B}=2500 \mathrm{G}$. (Gary, 2001).

Reynold's number compares the relative importance of the inertial versus viscous forces and thus allows to characterize flows as laminar $\left(R_{e}\right.$-small) or turbulent $\left(R_{e}\right.$-large $)$.

$$
R_{e}=\frac{U L}{v_{\mathrm{visc}}}
$$

Magnetic Reynold's number (also Lundquist number ) allows the comparison of the effects of magnetic advection and those of the magnetic resistive diffusion described by the first (3.18) and second term (3.18) on the right hand side of 3.13 , respectively. It 
is defined as a product of the typical velocity $\mathrm{U}$ and a typical length scale $\mathrm{L}$ of the flow divided by the magnetic diffusivity $\eta$ :

$$
R_{m}=\frac{U L}{\eta}
$$

\subsection{Ideal Adiabatic MHD Equations}

In order to study the coronal plasma, many assumptions have to be made. Ideal MHD is a set of simplified equations, including the MHD continuity equation (3.1), the momentum equation (3.25), Maxwell' s equations (3.26, 3.12, 3.28), and an adiabatic equation of state 3.24 , in the limit of no electric charges $\left(\boldsymbol{\nabla} \cdot \mathbf{E}=4 \pi \sigma_{Q}=0\right)$ and no electron diffusivity $(\eta=1 / 4 \pi \sigma \sim 0)$.

Assumptions:

- The plasma is highly collisional, so that the collisional time scales are much shorter compared to the MHD time scales and electrons and ions have a Maxwellian velocity distribution (A.6).

- Pressure isotropy

- Validity of ideal gas law:

$$
p_{g}=2 N_{e} k_{B} T
$$

- Validity of adiabatic equation of state:

$$
\frac{d}{d t} p \rho^{-\gamma}=0
$$

- Charge-neutrality $\nabla \cdot \mathbf{E}=0$ and $\nabla \cdot \mathbf{j}=0$

- The plasma is perfectly conductive: $R_{m}>>1$

In the momentum conservation equation(3.2), heat conduction, and viscous forces are neglected:

$$
\frac{\partial(\rho \mathbf{v})}{\partial t}+\nabla \cdot(\rho \mathbf{v} \mathbf{v})=-\nabla p_{\text {tot }}+\nabla \cdot \frac{\mathbf{B B}}{4 \pi}-\rho \mathbf{g} .
$$


In the equation of Ampère's circuital law (3.11), the electric field is assumed constant in time:

$$
\nabla \times \mathbf{B}=\frac{4 \pi}{c} \mathbf{j}
$$

Substituting $\mathbf{E}=0$ into the Ohm's law (3.12),

$$
\sigma(\mathbf{v} \times \mathbf{B})=\mathbf{j}
$$

and neglecting the diffusivity $(\eta=1 / 4 \pi \sigma \sim 0)$ in Faraday's law of induction 3.13 ,

$$
\nabla \times(\mathbf{v} \times \mathbf{B})=\frac{\partial \mathbf{B}}{\partial t} .
$$

Analogous to the Kelvin-Helmholtz theorem in ideal hydrodynamics, which states that vorticity lines move with the inviscid fluid, the ideal induction equation 3.28 yields the Alfvén's theorem of the frozen field lines (Alfvén 1942) :

In an ideal perfectly conductive plasma, the total amount of magnetic flux passing through any closed circuit moving with the local fluid velocity is constant in time. In other words, ideal MHD equations imply the conservation of magnetic flux and connectivity of the field lines.

\subsubsection{Force-free Equilibrium}

Coronal loops, arcades and quiescent prominences are often in magnetohydrostatic equilibrium, i.e. there is a balance between the external forces in the momentum equation 3.25 which reduces to:

$$
0=-\nabla p+\mathbf{j} \times \mathbf{B}-\rho \mathbf{g}
$$

In the limit of plasma- $\beta<<1$ (Fig. 3.1), thermal gas pressure can be neglected with respect to the magnetic pressure and gravity term, and the equation 3.29 can be re-written as:

$$
\mathbf{j} \times \mathbf{B}=\frac{1}{4 \pi}(\nabla \times \mathbf{B}) \times \mathbf{B}=0 .
$$

When no currants are present $(\mathbf{j}=0)$, the magnetic field is potential, i.e. it can be written as a gradient of a scalar function: $\mathbf{B}=-\nabla(\phi)$. When the currants are present $(\mathbf{j} \neq 0)$, the Lorenz force

$$
\mathbf{j} \times \mathbf{B}
$$


vanishes only if the currents flow along the magnetic field lines, thus forming helical flux structures. This condition can be written as

$$
\mathbf{j}=\alpha \cdot \mathbf{B},
$$

where $\alpha$ is a scalar function constant in time. The constant of proportionality $\alpha$ doesn't vary in space for linear force-free fields. Non-linear force-free fields assume $\alpha$ to generally vary with space, but stay constant along each field line. A non-potential force-free field contains more potential energy than the potential field with the same feet, but less than an unrestricted field in the photosphere.

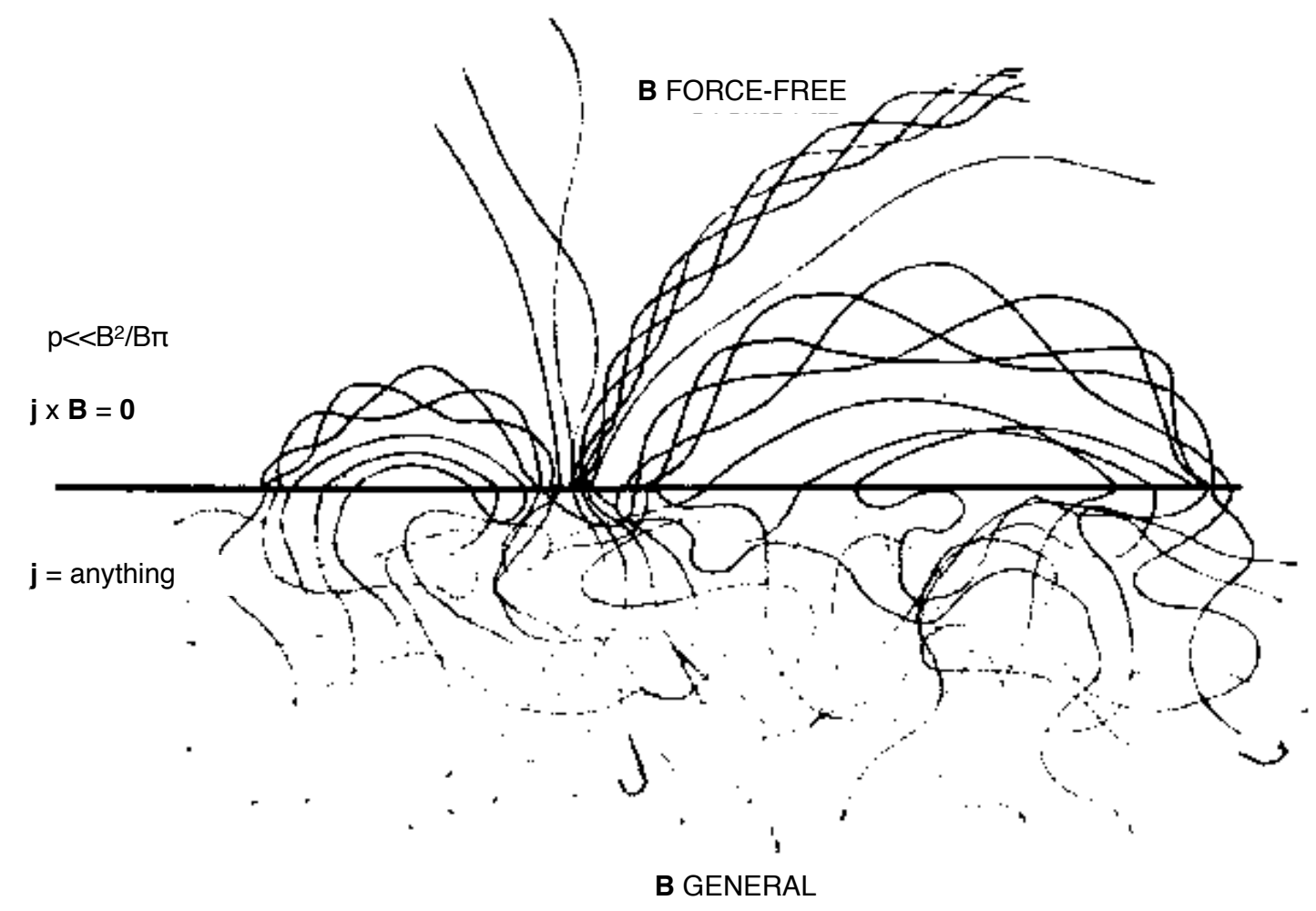

Figure 3.2: A cartoon describing the force-free configuration of the corona and the general field in the underlying photosphere adopted from Gold (1964)

The bottom part of the Fig. 3.2 from Gold (1964) describes the photosphere as a conductive medium, where the magnetic pressure (3.4) is negligible compared to the gas pressure $(\beta>>1)$ and therefore, the topology of the magnetic field is general. In contrast, the density of the conductive fluid drops, and the plasma is dominated by the 
magnetic pressure in the corona $(\beta<<1)$ so that the configuration of the field is forcefree, with helical flows along the magnetic field lines.

Differential rotation together with convective motions at the photosphere are wrapping up the coronal field, which is "frozen in", thus generating toroidal magnetic field, in the form of magnetic loops. As the magnetic stress is further accumulated due to the motion of the footpoints, the connectivity of the magnetic field lines breaks up via magnetic reconnection (4.1), in order to relax the system from a non-linear to a linear force-free field.

\subsection{Resistive MHD}

Although the solar corona plasma is generally a perfectly conductive medium, there are processes, such as magnetic reconnection (4.1), when the resistivity must be taken into account. Some of the approximations made in ideal MHD equations are valid also in resistive MHD approximations:

- Highly collisional plasma

- Pressure isotropy

- Validity of the ideal gas law $p_{g}=2 N_{e} k_{B} T$.

- Non-relativistic approximation $\mathrm{v}<<\mathrm{c}$

- Charge-neutrality $\nabla \cdot \mathbf{E}=0$ and $\nabla \cdot \mathbf{j}=0$

- Diffusivity cannot be neglected $R_{m} \ngtr 1$

Resistive energy equation becomes:

$$
\frac{1}{\gamma-1} \frac{D p}{D t}+\frac{\gamma}{\gamma-1} p \nabla \cdot \mathbf{v}+\frac{\eta}{4 \pi}(\nabla \times \mathbf{B})^{2}=-Q_{\text {visc }}-Q_{\text {rad }}-Q_{c}
$$

The second term (3.19) in the induction equation (3.13) cannot be neglected due to the finite resistivity $\eta$ and it is not assumed $\mathbf{E} \neq 0$ in Ohm's law.

\subsubsection{MHD in the diffusive limit}

For values of the magnetic Reynold's number $R_{m}<<1$, the convective term (3.18) can be neglected with respect to the diffusive term $(3.19)$ in 3.13 , yielding a diffusion equation: 


$$
\frac{\partial \mathbf{B}}{\partial t}=\eta \nabla^{2} \mathbf{B}
$$

Substituting an inverse of a typical length scale $L^{-1}$ and time scale $\tau_{\text {diff }}^{-1}$ instead of the derivations $\nabla$ and $\partial / \partial t$ gives the relation:

$$
\tau_{\mathrm{diff}}=L^{2} / \eta \quad[\mathrm{s}],
$$

stating that the magnetic field variations occurring on a length scale $L$ dissipate on a time-scale $\tau_{\text {diff. }}$ It follows from Eq. (3.34) that the dissipation of magnetic fields is faster for smaller length-scales. In a fully ionized plasma $\eta^{-1}=4 \pi N_{e} e^{2} / m_{e} v_{c} \approx$ $10^{-9} T^{3 / 2} \mathrm{~s} \mathrm{~cm}^{-2}$, and the frequency of collisions is given by Eq. 3.17), yielding diffusive times of the order of $\tau_{\text {diff }}=L^{2} 10^{-9} T^{3 / 2} \mathrm{~s}$. 



\section{Solar Eruptions}

Magnetic energy stored in the solar corona in the form of electric currents is currently accepted as the only possible source for the energy release during solar flares (4.2). Theoretical flare models have been developed in order to understand how the currents are formed and dissipated. To date, magnetic reconnection (4.1) is the only suggested mechanism which can explain observations of the apparent motion of the flare ribbons. Based on the initial geometry, there are three main flare models assuming rapid reconnection, which are able to store and release magnetic energy:

- Emerging-Flux Model

- Sheared-Arcade Model

- Magnetic-Flux-Rope Model

The flares discussed in the Sections (6.3) and (6.4) were accompanied by filament eruptions (4.3) and CMEs (4.4). CME associated flare models explaining how the magnetic flux is ejected into the interplanetary space during a flare will be discussed in the Section (4.4).

\subsection{Magnetic Reconnection}

The physical mechanism, which was only later named magnetic reconnection by Dungey (1953), was first suggested by Giovanelli (1946) as a plausible mechanism for particle acceleration in solar flares. Magnetic reconnection involves a change in connectivity of magnetic field lines as a response to excessive stress. Although it has been widely studied (Giovanelli 1948, Dungey 1953, Cowling 1953, Sweet 1958, Parker 1963, Sweet 1969, Petschek 1964), the basic physics of the mechanism hasn't been established so far. In the solar interior, it is responsible for the generation of magnetic field in the dynamo process. In the photosphere, reconnection takes place in the interface between the pre-existing flux and the emerging flux of opposite polarity. In the chromosphere, magnetic reconnection occurs during magnetic flux emergence, flux cancellation and during explosive events. In the corona, it may involve either quasi-steady changes, small scale 
events which may play a role in coronal heating, or sudden violent processes such as flares and CMEs, on which the emphasis will be laid on in this thesis.

Magnetic reconnection is a non-ideal process, governed by the resistive induction equation (3.13). It can occur only if Alfvén's theorem of the frozen field lines (3.28) is violated and the plasma can flow across the magnetic field lines. The topological changes due to magnetic reconnection are accompanied by a release of the free nonpotential energy, which is mostly converted into the plasma heating and acceleration of particles and ions.

When two oppositely directed magnetic flux systems are pushed together, the magnetic field has to drop to zero at the boundary in order to allow for a continuous change from a positive to a negative magnetic field strength. Due to the balance of the total pressure across the neutral boundary layer,

$$
p_{\mathrm{g}, \mathrm{ext}}+B_{\mathrm{ext}}{ }^{2} / 8 \pi=p_{\mathrm{g}, \mathrm{int}}+B_{\mathrm{int}}^{2} / 8 \pi,
$$

the thermal pressure in the neutral layer with a zero magnetic pressure (Eq. 3.4) must be higher than the thermal pressure $p_{g}$ on both sides of the boundary, where the magnetic field strength is finite. Hence, near the neutral layer, the magnetic field gradient $(\nabla B)$ is large, and so is the resistive term (3.19) in the induction equation 3.18, the field lines are no more frozen and their breaking and reconnecting may take place.

The rate of the reconnection can be expressed in terms of the ratio of inflow $\mathrm{v}_{\text {in }}$ to outflow speed $\sim c_{A}$ (5.2), which corresponds to the Alfvén Mach number of the inflow $M_{\text {in }}$. The continuity equation (3.1) yields the conservation of mass, i.e.

$$
\mathrm{v}_{\text {in }} \Delta_{\mathrm{d}} \approx \mathrm{c}_{\mathrm{A}} \mathrm{L}_{\mathrm{d}},
$$

where $\Delta_{d}$ and $L_{d}$ are the width and length of the diffusion region respectively. The inflow velocity $\mathrm{v}_{\text {in }}$ can be expressed in terms of diffusion velocity given by

$$
\mathrm{v}_{\text {in }}=\eta / \Delta_{\mathrm{d}} .
$$

Taking the product of the equations (4.2) and (4.3), and inserting the magnetic Reynold's number $R_{m}$ for the expression $C_{A} L / \eta$, the following estimates of Mach number $M_{\text {in }}$ and ratio of the diffusion region length to width can be found (Parker 1973):

$$
\begin{gathered}
M_{\text {in }}=\frac{\mathrm{v}_{\text {in }}}{c_{A}}=\frac{1}{\sqrt{R_{m}}}, \\
\frac{L_{d}}{\Delta}=\frac{1}{\sqrt{R_{m}}} .
\end{gathered}
$$




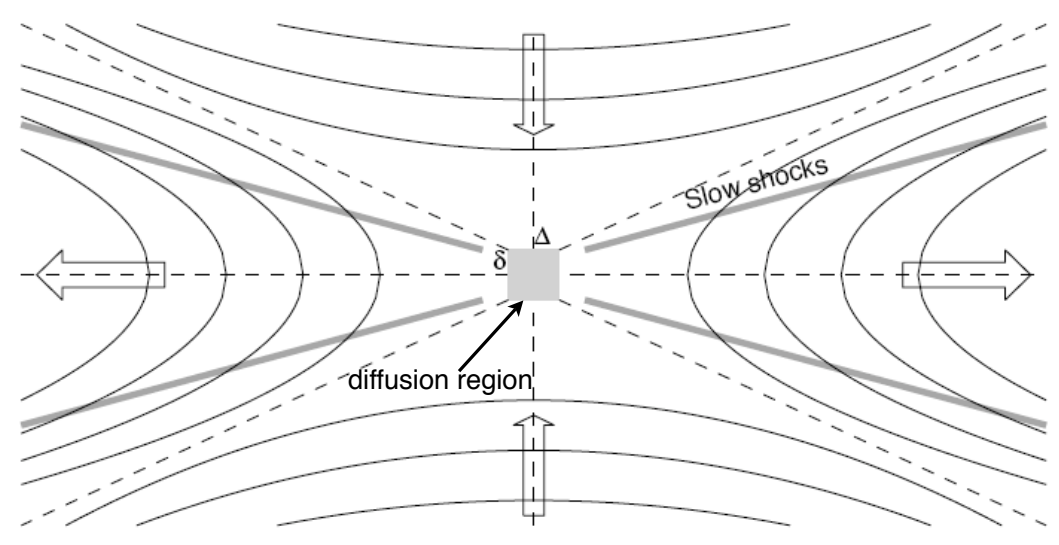

Figure 4.1: Petschek type reconnection in two dimensions (Petschek 1964). Grey area corresponds to the diffusion region.

Petschek type reconnection shown in Fig. 4.1 is faster than the previously suggested Sweet-Parker mechanism (Sweet 1958, Parker 1963), whose diffusion region (region colored grey in Fig. 4.1) is much more elongated than it is wide.

\subsection{Flares}

Solar flares are powerful explosions on the Sun releasing energies up to $\sim 10^{32} \mathrm{erg}$ characterized by an increased emission in the bands ranging from visible to radio wavelengths. They involve the reconnection of large systems of magnetic flux on the Sun rapidly releasing energy stored in the magnetic field over a period of hours to days. The full-Sun X-ray flux monitor GOES (Geostationary Operational Environmental Satellite) provides classification of the observed solar flares based on their peak X-ray intensities. Classification using letters ( $\mathrm{A}, \mathrm{B}, \mathrm{C}, \mathrm{M}$ and $\mathrm{X}$ ) is logarithmic with a span of five orders of magnitude, the most energetic $\mathrm{X}$ class flares having a peak flux of the order of $10^{-4} \mathrm{ergs}^{-1} \mathrm{~cm}^{-2}$. Each class denoted by a letter is then linearly subdivided into classes 1-9, where the number of a given subclass expresses how many times it is more powerful than the class number one. Flares are often closely associated with CMEs, which led to their interpretation as the primary trigger mechanism of CMEs and geomagnetic storms. Observations of CMEs preceding a solar flare (Zhang et al.2001) and those without an associated flare, as well as the lack of high resolution observations and therefore accuracy in the estimation of the onset of the CMEs (Kahler 1992) have put the hypothesis of the flare being the cause of the CME in doubt (see paragraph 4.4.2.3). 


\subsubsection{Flare Emission}

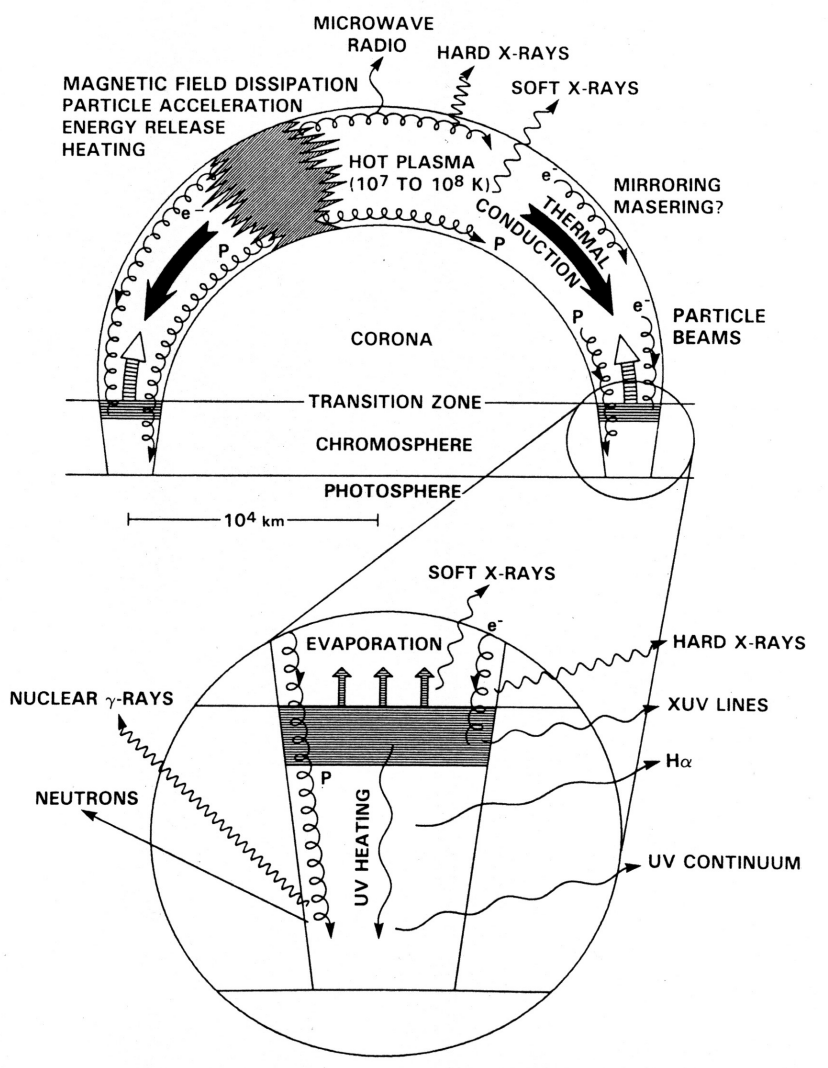

Figure 4.2: Cartoon by Joe Gurman (Dennis et al. 1986, Dennis \& Schwartz 1989) showing the variety of emissions produced at different parts of a flare loop.

\subsubsection{1 $\gamma-$ Rays and X-Rays}

High energy particles interacting with the dense chromosphere can produce $\gamma-$ and Xrays with photon energy in the range $\sim 10-100 \mathrm{keV}$. The exact mechanism involved, as well as the wavelength of the emitted photons depend on the energy of the colliding particles. Possible particle acceleration mechanisms generating the energetic electrons include direct acceleration by electric fields present in the reconnection current sheets, wave-particle interaction involving trapping of electrons by waves and turbulence and shock fronts.

\section{Gamma rays}

$\gamma$ - rays result from the decay of elements in the chromospheric footpoints (Fig. 4.2) 
such as carbon, nitrogen, oxygen, etc., which were excited to high energy states in nuclear interactions with highly relativistic electrons accelerated in flares. Therefore, $\gamma$-rays may serve as a tool for the density diagnostic of the relativistic particles accelerated in flares as well as the target nuclei in the chromosphere.

\section{Hard X-rays}

HXR are observed at the loop tops and footpoints at the level of the transition region and chromosphere (Fig. 4.2). They are produced in collisions between the mildly relativistic electrons and thermal ions, therefore they carry information on particles energized during flares and serve as a diagnostic on the acceleration, propagation and trapping of the electrons. Among processes in which hard X-rays are formed are thermal bremsstrahlung, thick-target bremsstrahlung (Brown 1971) and thin-target bremsstrahlung. A more detailed description of HXR sources in association with with solar flares will be given in the Section 4.2.1.2. Krucker et al. (2008) reviews HXR emission observed with the Reuven Ramaty High Energy Solar Spectroscopic Imager (RHESSI) during various stages of flares.

\section{Soft X-rays}

The bulk of the radiative energy of the coronal plasma at temperatures above $1 \mathrm{MK}$ is concentrated in the soft X-ray range (Fig. 4.2), in which the flare loops together with their footpoints are very well seen. SXRs are produced as a consequence of collisional excitation and thermal bremsstrahlung (see Section 2.2.2.1) - they involve electrons with a Maxwellian velocity distribution (A.6). Synthetic spectra relevant for an active region and a flare are shown in Fig. (4.3) and (4.4). 


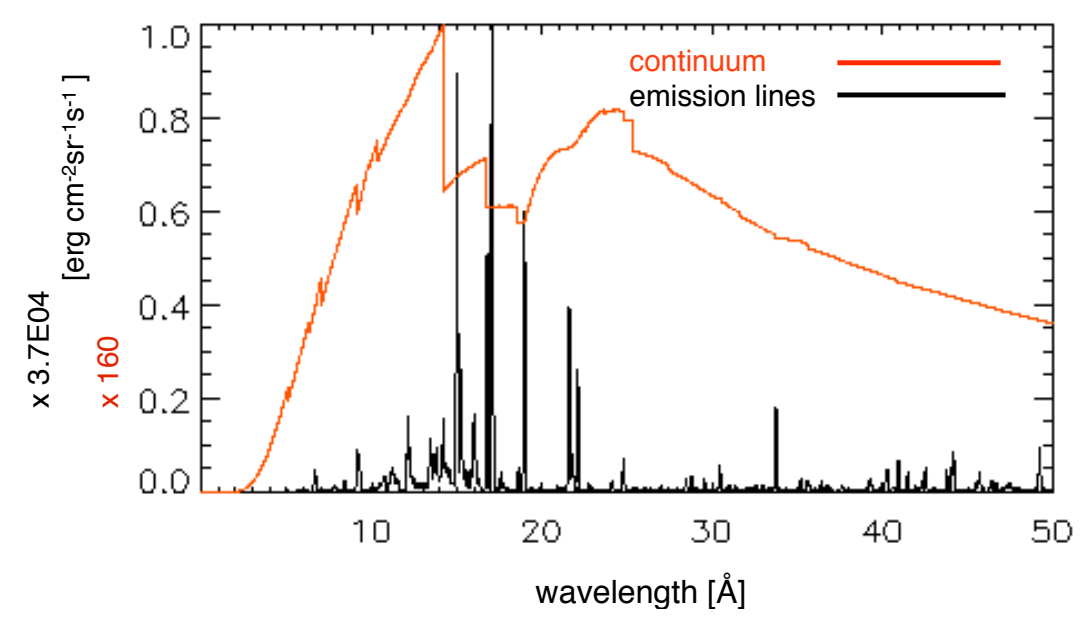

Figure 4.3: Synthetic SXR spectrum obtained using CHIANTI atomic database containing emission lines (black) superimposed on continuum (red) assuming ionization equilibrium after Mazzotta et al.(1998) and an active region DEM.

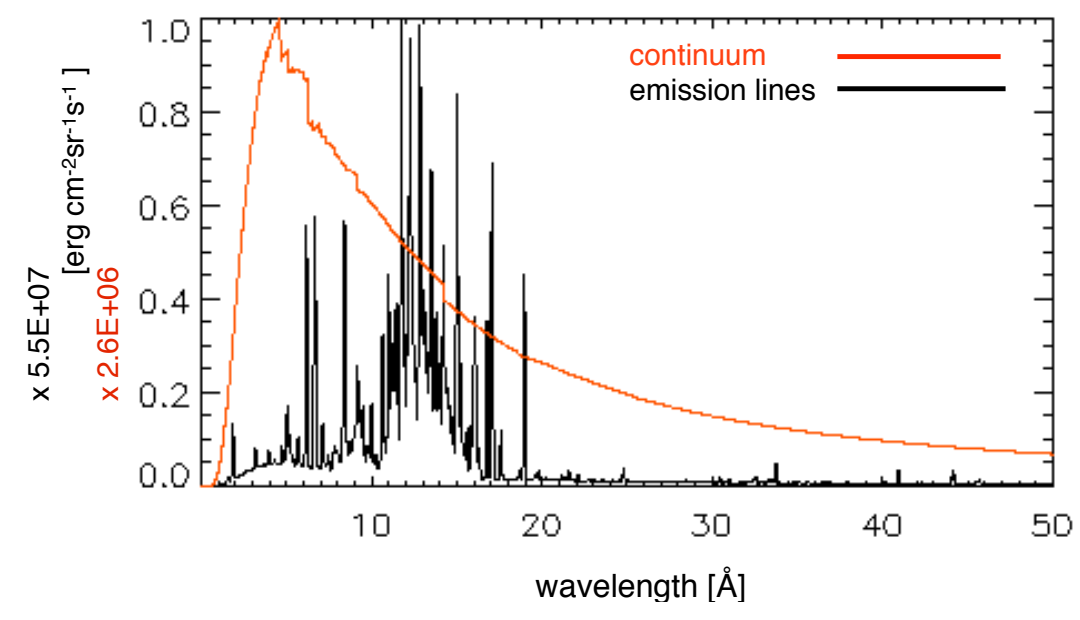

Figure 4.4: Synthetic SXR spectrum obtained using CHIANTI atomic database containing emission lines (black) superimposed on continuum (red) assuming ionization equilibrium after Mazzotta et al. (1998) and a flare DEM.

\subsubsection{Flare Evolution in $\gamma$ and X-Rays}

The development of flares goes through three main stages: 


\section{Pre-flare phase}

The first is the pre-flare stage, in which the soft X-ray emission gradually increases with no significant increase in hard $\mathrm{X}$ - and $\gamma$ - rays suggesting plasma heating.

\section{Impulsive phase}

The flare itself starts with the "impulsive phase", which is characterized by a rapid increase in intensity of $\gamma$ - rays, SXRs and HXRs.

\section{- Footpoint HXR source}

According to the standard model - the so-called "thick-target model" (Brown 1971, Hudson 1972), the strongest HXRs are emitted when non-thermal electrons and ions, injected near a flare loop top, precipitate to the dense chromosphere to heat up the plasma at the footpoints (Fig. 4.5 b-d) of the flare loops. Heating is due to the loss of kinetic energy of the relativistic electrons in Coulomb and ionizing collisions. The first observations of the HXR footpoint emission was made with the Solar Maximum Mission (Hoyng et al. 1981).

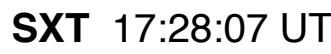

HXT 17:27:35 - 17:28:15 UT

$14-23 \mathrm{keV}$

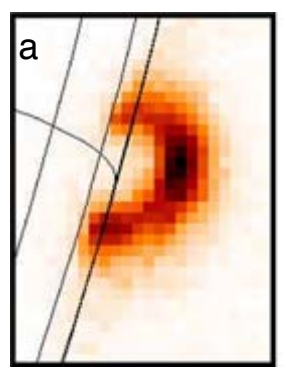

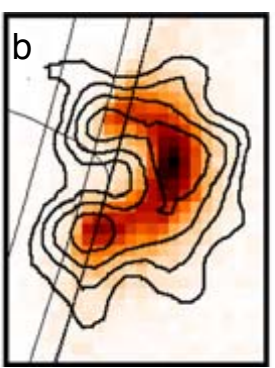

23-33 keV

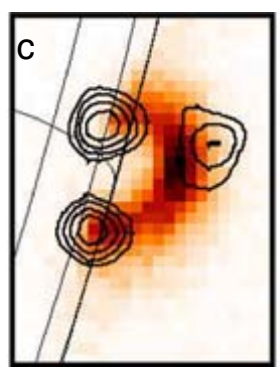

33-53 keV

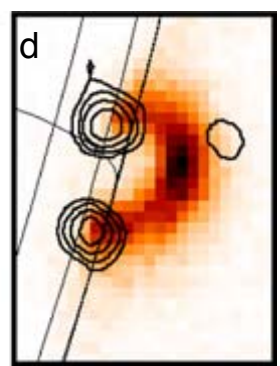

Figure 4.5: HXR emission (black contours) overlaid on an SXR image during the "Masuda Flare" on 13 January 1992. The SXR image was taken with the Yohkoh/SXT at 17:28:07 UT. The HXR (Yohkoh/HXT) contours in b-d correspond to three different energy bands taken with Yohkoh/HXT from 17:27:35 - 17:28:15 UT. The contour levels are $6.25,12.5,25.0$ and $50.0 \%$ of the peak value. Courtesy of Krucker et al. (2008).

\section{- Masuda HXR source}

Non-thermal bremsstrahlung by electrons accelerated and trapped in the cusp region due to magnetic mirroring or by wave turbulence in the reconnection outflow produces a HXR above the loop top source (Fig. $4.5 \mathrm{c}$ and 
d) discovered by Masuda et al. (1994). It had a single peak that lasted for about 2 minutes. At the location of the Masuda loop top HXR source, no enhancement in thermal emission was observed, indicating low plasma density (Hudson \& Ryan 1995).

\section{- Double coronal HXR sources}

Sui \& Holman (2003) and Sui et al. (2004) observed double coronal sources early in the impulsive phase of the homologous flares that occurred during 14-16 April 2002 (Fig. 4.6). The temperature gradients are of the opposite signs at the location of both sources (Sui and Holman 2003). The inverted gradients were interpreted as evidence for a current sheet formed between the tops of the flare loops and the coronal sources above the loops (Sui et al. 2004).

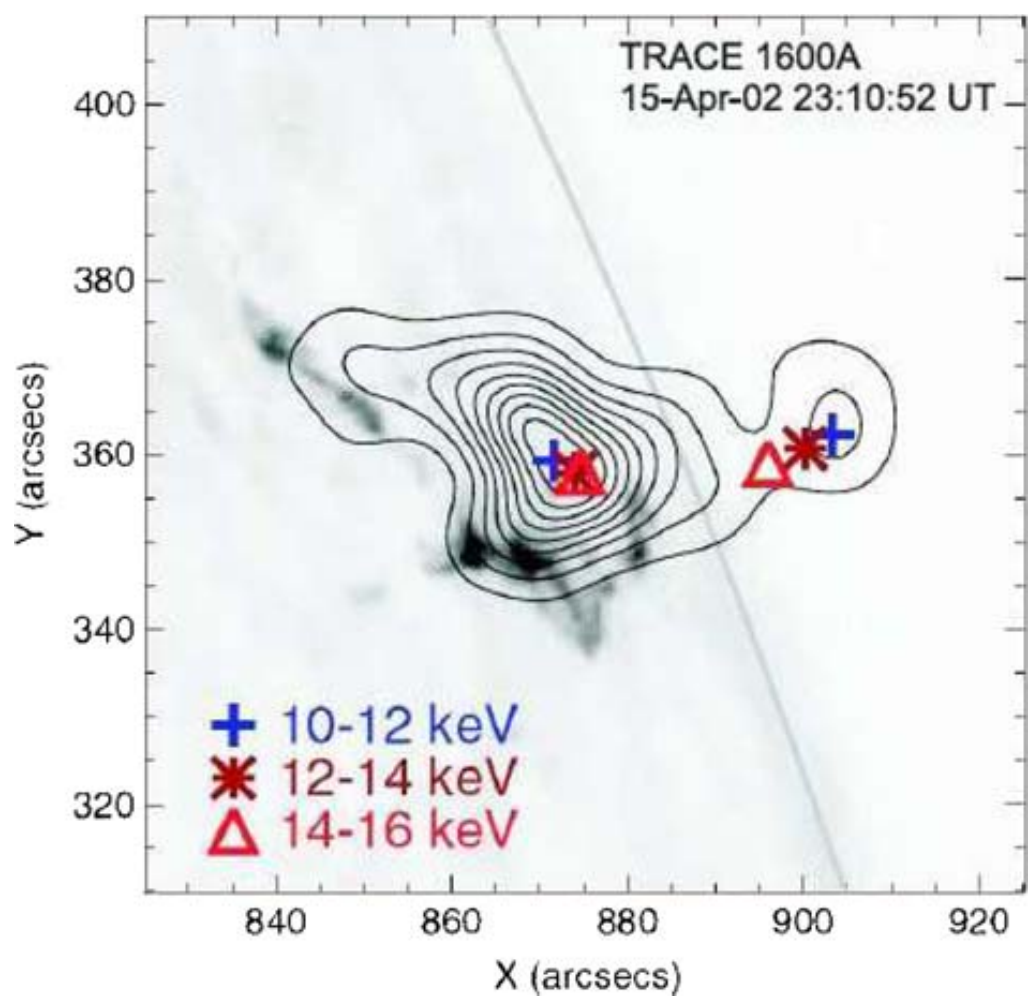

Figure 4.6: TRACE $1600 \AA$ image overlaid with X-ray contours obtained from RHESSI in the energy range 10-12 keV during the flare on 15 April 2002, revealing thermal emission from the flare loops and from the coronal source above.

\section{Gradual phase}

During the "gradual phase", postflare loops form, with a characteristic cusp shape that traces out the relaxed dipole-like magnetic field lines. At their footpoints, 
the overpressure of the heated plasma forms upflows seen in soft X-rays in the postflare loops in a process named chromospheric evaporation. The HXR- and $\gamma-$ ray flux starts to decay.

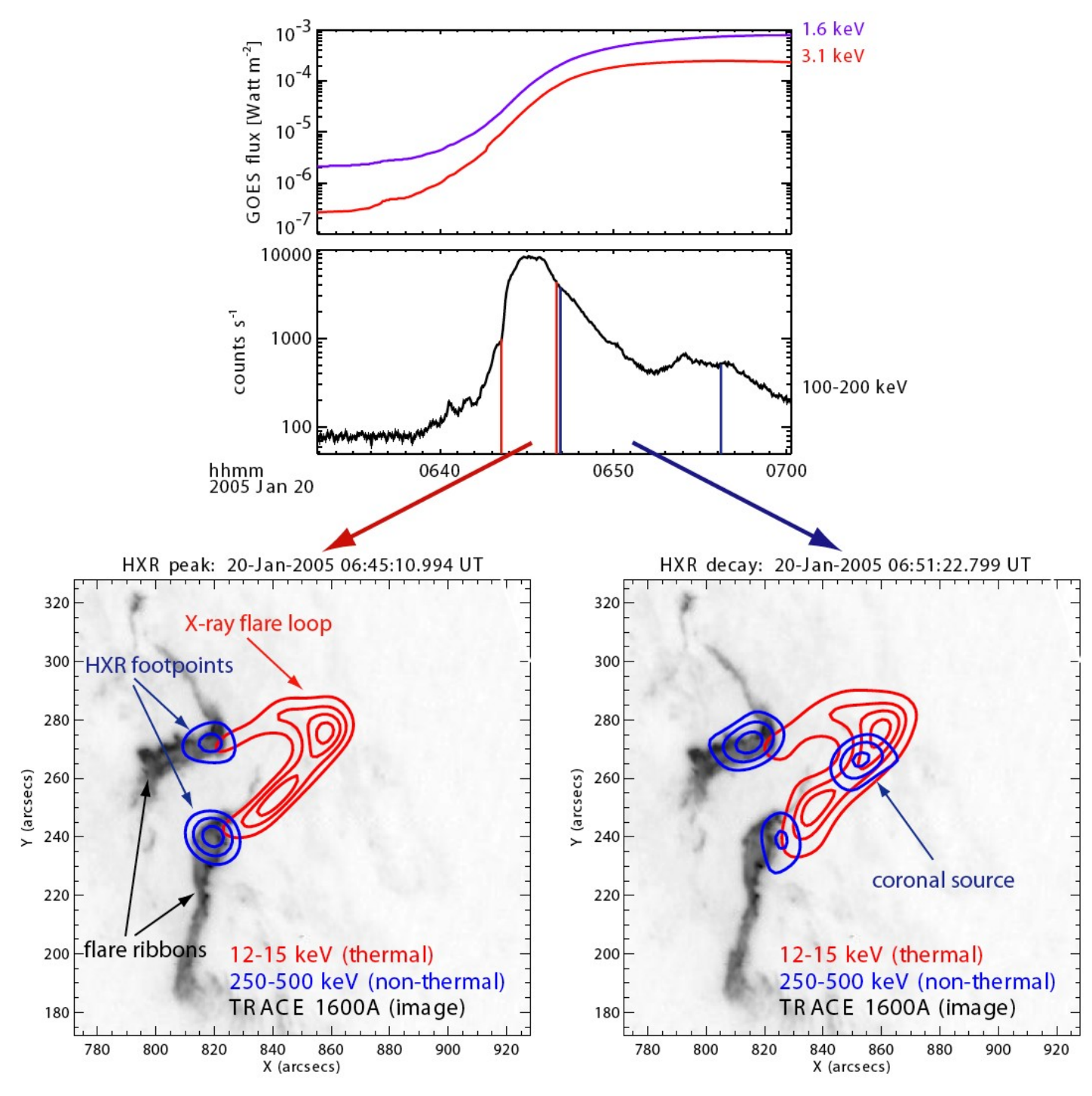

Figure 4.7: X-ray observations of an X-class flare that produced the biggest solar proton storm ever observed.

Top: 1-minute averages of soft X-ray flux from GOES satellite.

Middle: HXR counts

Bottom: TRACE $1600 \AA$ with RHESSI contours. 


\subsubsection{Ultraviolet (UV) Emission}

In addition to the HXRs observed at the flare foot-points, the flare ribbons, which move apart at a velocity highly correlated with the reconnection rate, are also recognized as bright, compact sources of UV continuum (TRACE at $1600 \AA$ ), white light and $\mathrm{H} \alpha$ in the lower atmosphere (Fig. 4.7). They lie along sites where electrons accelerated during the flare interact with the chromospheric plasma.

Skylab observations of three flares made by Cheng \& Rosenberg (1976) have shown that the enhancement in intensities of transition region lines during flare maximum is significantly higher in comparison with the chromospheric lines. Moreover, transition region lines are broadened and initially predominantly red-shifted.

Spectroscopic observations of the solar flares discussed in the Chapter (6) have been made in the transition region line Si III $1113 \AA$, coronal line Ca x 1115/2 $\AA$ and the flare line Fe XIX $1118 \AA$. They are produced in dipole transitions due to the electron excitation. The $\mathrm{Ca} x$ and Fe xIx lines come from plasma that emits also in the SXR domain. They therefore complement SXR observations, as they allow to determine Doppler shifts of flows.

\subsubsection{Radio Emission}

Flares produce radio emission in the range $\sim 20-400 \mathrm{MHz}$.

Incoherent emission in the corona with a continuous distribution is produced due to circular motion of the electrons around the magnetic field lines. If the motion is nonrelativistic, the emission mechanism is called gyroresonance or cyclotron emission. Mildly/highly relativistic particles produce gyrosynchrotron/synchrotron emission.

Solar radio bursts are a signature of electron beams accelerated in flares and propagating along the magnetic field lines. They are due to plasma emission, a coherent mechanism resulting from the resonant wave-particle interactions of beams of electrons propagating along the magnetic field lines.

\section{Thermal Bremsstrahlung}

Given the same formation mechanism, microwave free-free continuum emission in flare loops is highly correlated to the emission in SXR. 

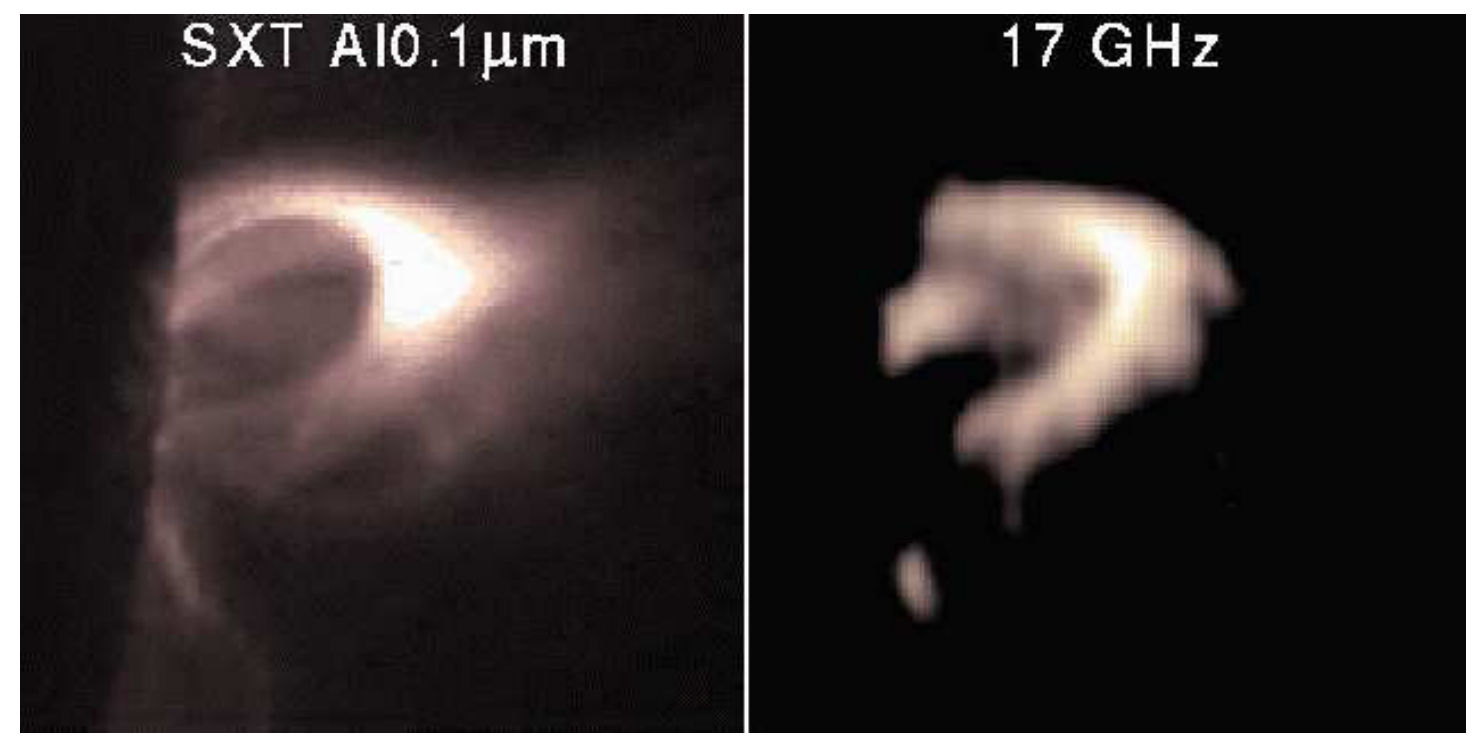

Figure 4.8: Flare on Mar 161993 taken with Yohkoh/SXT (left) and Nobeyama Radioheliograph at $17 \mathrm{GHz}$ (right). From Hanaoka (1994).

\section{Gyrosynchrotron Emission}

Gyrosynchrotron emission in microwaves (4.2) is produced when trapped relativistic electrons interact with the waves. Its light curve is highly correlated to the time evolution of HXRs formed when the relativistic electrons interact with the dense chromosphere. The peaks of the microwaves lag behind the HXR peaks due to the time needed for trapping of the electrons.

\section{Type II bursts}

A Type II burst (Fig. 4.9 ) is produced at the plasma frequency $f_{p}$ and $2 f_{p}$ by nonlinear processes involving Langmuir waves, driven by the electron beams accelerated at the shocks and in current sheets. The plasma frequency $f_{p}$ in CGS units is given by:

$$
f_{p} \approx \sqrt{N_{e} e^{2} / \pi m_{e}} \approx 8980 \sqrt{N_{e}} \quad[\mathrm{~Hz}] .
$$

So, for the electron density in the range $10^{7} \mathrm{~cm}^{-3}<\mathrm{N}_{\mathrm{e}}<10^{9} \mathrm{~cm}^{-3}$ as is assumed in the lower corona, the corresponding plasma frequency is $30 \mathrm{MHz} \lesssim \mathrm{f}_{\mathrm{p}} \lesssim 280 \mathrm{MHz}$.

Type IIs are a signature of the reconnection process and shock waves. As Type II bursts slowly drift down in frequency as the shock propagates towards lower density plasma, they carry information on the shock's propagation velocity if the density structure of the corona is known. The frequency at which they are observed may serve as a diagnostic of the density (Wild 1950, Nelson \& Melrose 1985).

An example of a Type II burst preceded by 3 solar Type III bursts is shown in the dynamic spectrum from the Green Bank Solar Radio Burst Spectrometer in Fig. 4.9 
a. Below (dotted line in Fig. 4.9 b), the corresponding light curve of the SXR flux recorded with the GOES satellite reveals a $\mathrm{C}$ class flare. The solid line is the dynamic spectrum from (Fig. 4.9 a) averaged over all the frequencies. Assuming a known density structure of the corona, $N_{e}=N_{e}(r)=\left(f_{p}(r) / 8980\right)^{2}$, the shock propagation velocity $\mathrm{v}=\mathrm{dr} / \mathrm{dt}=\partial \mathrm{r} / \partial \mathrm{f} \cdot \partial \mathrm{f} / \partial \mathrm{t}$ can be determined from the slope $\partial f / \partial t$ of the type II burst, whose negative sign suggests an outward moving source.

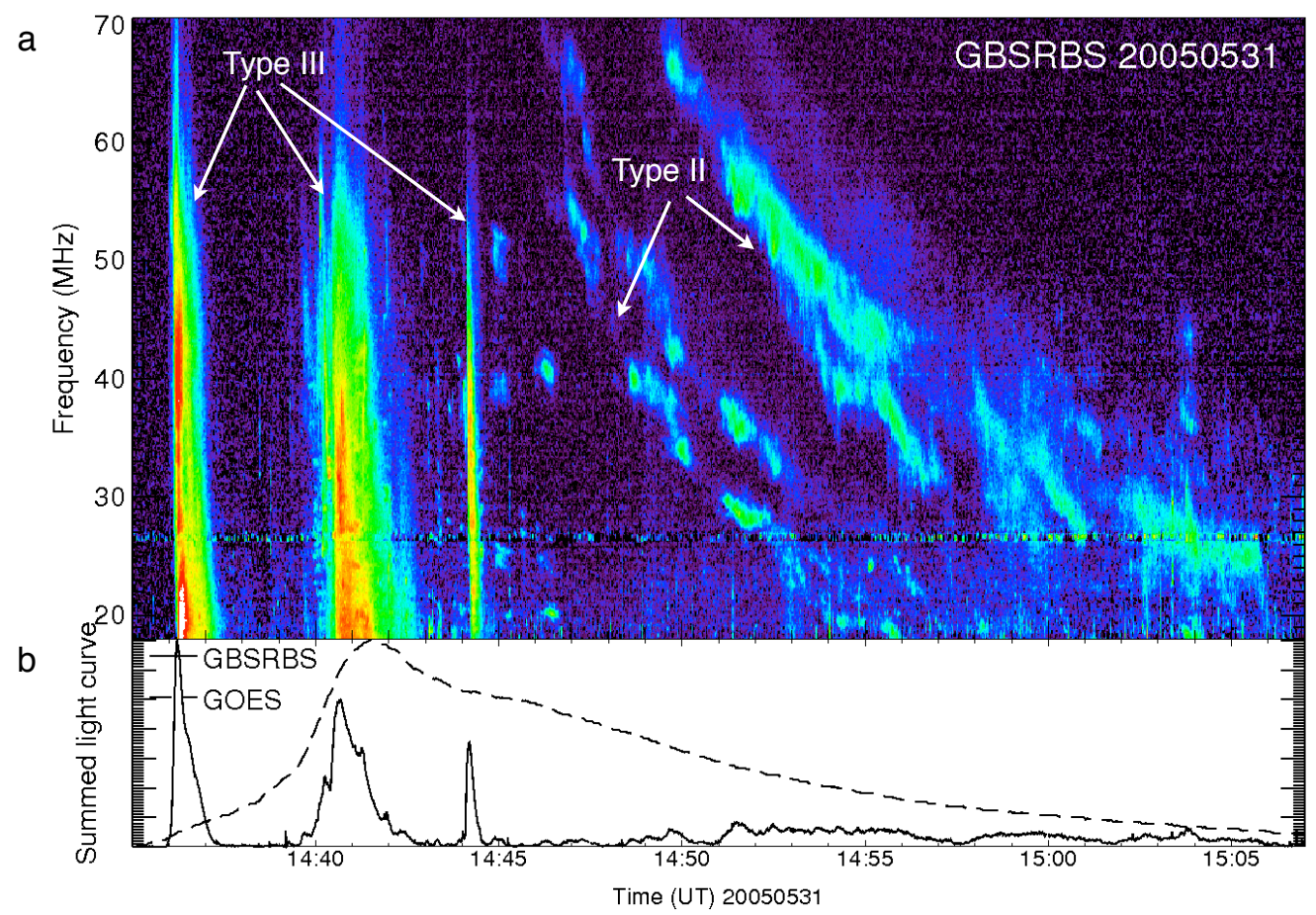

Figure 4.9: 3 Solar radio Type III bursts followed by a Type II (Courtesy http://gbsrbs.nrao.edu/)

\section{Type III bursts}

Type III bursts are due to relativistic electron beams propagating upward. They can be recognized from the steep negative frequency-time drift in Fig. 4.9 a). They are produced immediately after the flare onset (within several minutes), together with HXR emission, as a reaction to the formation of electron beams with a non-Maxwellian distribution characterized by a slope $\partial f / \partial \mathrm{v}_{\|}>0$. The correlation between the $\mathrm{C}$ class flare and the Type III bursts is well seen in Fig. 4.9 a.

Loss-cone Emission The mirroring of the electrons in flare loops gives rise to losscone velocity distributions with a slope positive in the direction perpendicular to the 
magnetic field lines $\partial f / \partial \mathrm{v}_{\perp}>0$ which produce cyclotron maser emission (Fig. 4.2 , labeled mirroring, MASERing).

\subsection{Eruptive Prominences}

Cool $\left(\sim 10^{4} \mathrm{~K}\right)$, dense $\left(N_{e} \sim 10^{10} \mathrm{~cm}^{-3}\right)$ structures of different shapes immersed in the hot ( $\gtrsim 1 \mathrm{MK})$, sparse $\left(N_{e} \sim 10^{8} \mathrm{~cm}^{-3}\right)$ corona, are called prominences when observed in emission on the solar limb, and filaments when seen as absorption on the solar disk. They are often observed above magnetic polarity inversion lines. They seem to be supported by a strongly sheared and twisted magnetic field (Mackay et al. 2010) which represents a plausible storage for the magnetic energy. The supporting magnetic field loops can remain quiescent for a very long time before they erupt. The relationship between prominence eruptions, CMEs and other forms of solar activity such as active regions, sunspots, solar flares has been studied since the first detection of CMEs in the 1970s (Gosling et al. 1974). 


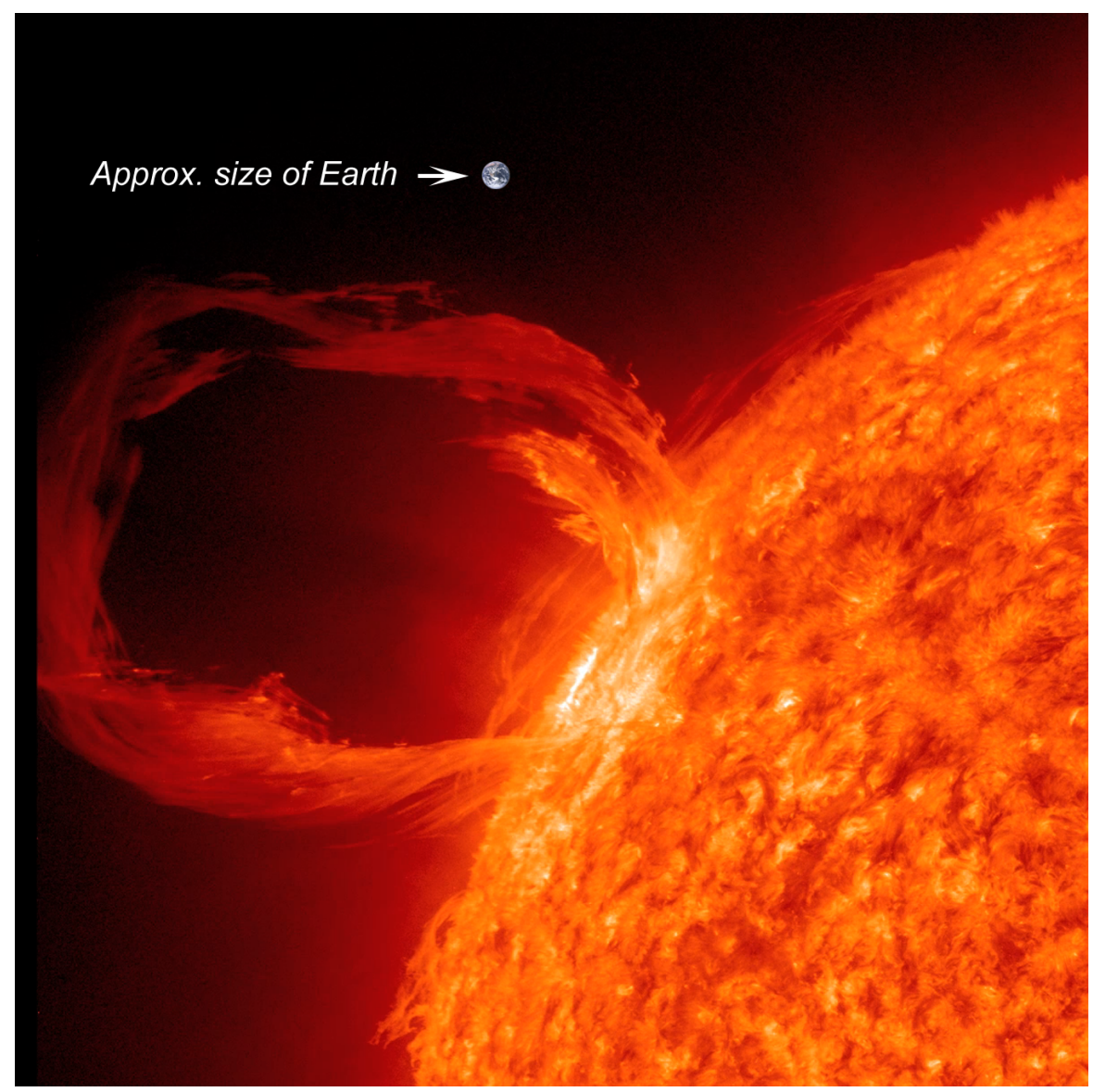

Figure 4.10: Chromosphere and a large prominence as seen in He II $304 \AA$ by AIA on board of the Solar Dynamics Observatory.

\subsubsection{EUV Observations of Prominences}

Although the prominences are clearly visible as an enhancement in chromospheric lines, they can also be observed as dark structures in coronal lines. If cool prominence gas is present, the coronal radiation of the iron lines Fe IX $171 \AA$, Fe XII $195 \AA$, and Fe Xv 284 $\AA$ may be reduced due to coronal volume blocking or/and via partial absorption mainly by $\mathrm{H}_{\mathrm{I}}, \mathrm{He}$ I and $\mathrm{He}$ II . The significance of each mechanism in contributing into the darkening of the EUV lines depends on the filament's extension along the line of sight - i.e. on its geometry with respect to the observer, and on the EUV opacity - i.e. on filament's temperature, electron density, $\mathrm{H} \alpha$ and $\mathrm{He}$ abundance and ionization states.

Volume blocking The corona is generally optically thin, therefore the observed emission comes from the plasma in a long column along the line-of-sight. Any present 
chromospheric material pushes the coronal plasma out of our view thus the integrated emission of the observed column is lower compared to the ambient corona.

Absorption Absorption features in coronal EUV lines have been discovered by Skylab (Orrall \& Schmahl 1976). The degree of weakening of a line with a wavelength $\lambda$ due to absorption can be represented using the optical thickness $\tau$ (A.5):

$$
I_{\mathrm{obs}}(\lambda) / I_{\mathrm{th}}(\lambda)=e^{-\tau(\lambda)} .
$$

For wavelengths below the Hydrogen Lyman continuum head $(\lambda<912 \AA)$, the absorption takes place via Lyman continuum absorption in neutral Hydrogen $\mathrm{H}$ I. At wavelengths $\lambda<504 \AA$, the absorption by neutral Helium, and for $\lambda<228 \AA$, also by singly ionized Helium occur:

$$
\tau=\sigma_{\mathrm{HI}} N_{\mathrm{HI}}+\sigma_{\mathrm{HeI}} N_{\mathrm{HeI}}+\sigma_{\mathrm{HeII}} N_{\mathrm{HeII}},
$$

where $N_{\mathrm{HI}, \mathrm{HeI}, \mathrm{HeII}}$ are the integrated (column) densities of the given ions along the lineof-sight and $\sigma_{\mathrm{HI}, \mathrm{HeI}, \mathrm{HeII}}$ are theirs photoionization cross-Sections.

Anzer \& Heinzel (2005) have shown that the continuum opacity of the EUV Fe lines is comparable to that of the $\mathrm{H} \alpha$ line and therefore any prominence visible in $\mathrm{H} \alpha$ line must be detectable as absorption in the iron lines as well. On the other hand, the absorption is negligible if a prominence shows no observable enhancement in $\mathrm{H} \alpha$ line. Lines above the Lyman continuum head are not affected by absorption, therefore any decrease in lines with $\lambda>912 \AA$ must be exclusively due to volume blocking.

\subsection{Coronal Mass Ejections}

CMEs are transients of coronal mass of $\sim 10^{13} \mathrm{~kg}$ moving away from the Sun with velocities ranging from $200-3000 \mathrm{~km} \mathrm{~s}^{-1}$. They are a fairly common phenomenon whose frequency varies with the solar cycle, ranging from $\sim 1$ occurrence in 2 days at the minimum to a few per day.

\subsubsection{CME Observations}

CMEs are most frequently seen in coronagraphic white light images which block the bright photospheric light in order to bring up the emission due to Thompson scattering on electrons. Signatures in other wavelength bands include $\mathrm{H} \alpha$ and $\mathrm{He}$ II $304 \AA$ prominence eruptions (see 4.3), EUV dimming corresponding to the footpoints of the CMEs as well as the hot X-ray cusp shaped post-flare loops and arcades formed under erupted CMEs. 


\subsubsection{Theoretical Models of CME Initiation}

A large number of theoretical concepts of the initiation of CMEs has been proposed over the years with attempts to explain the above mentioned signatures. They were summarized by Klimchuk (2001). A variety of cartoons describing the general models as well as the special events has been collected by Hudson and can be found $<$ here. $>$. A CME occurs when the balance of the forces maintaining the system in equilibrium breaks down and the gradient of the pressure $-\nabla\left(p+B^{2} / 8 \pi\right)$ responsible for the expansion overcomes the downward-directed magnetic tension force

$$
(1 / 4 \pi)(\mathbf{B} \cdot \nabla) \mathbf{B} \text {. }
$$

Due to the low $\beta$-plasma in active regions, the gravity force and gas pressure are often omitted in the CME models. It is generally accepted that the CME onset mechanism involves a release of free magnetic energy associated with electric currents present in the corona since the kinetic, thermal and gravitational energy densities are not sufficient. The exact mechanism involved in the release of the energy stored in the magnetic field though remains unknown and is the key question to be answered by the models.

\subsubsection{Pre-Eruptive Topology}

The topology of the pre-eruptive magnetic field in most of the models is initially flux rope-like or arcade-like (Fig. 4.11).

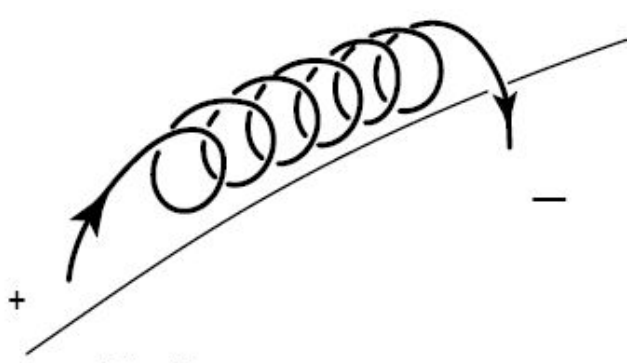

(a)

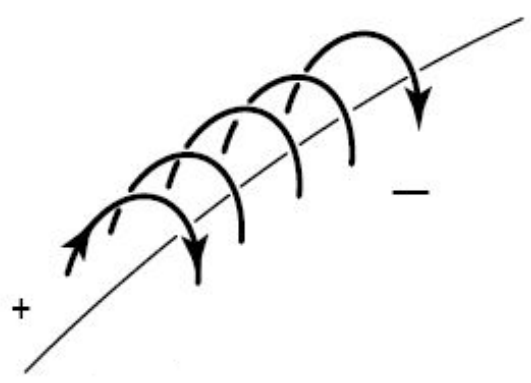

(b)

Figure 4.11: Flux-rope (a) and arcade (b) configuration (Klimchuk 2001). The solid line represents the polarity inversion line. The twist of the flux rope (a) is exaggerated for the purpose of representation. 


\subsubsection{Magnetic Flux-Rope Model}

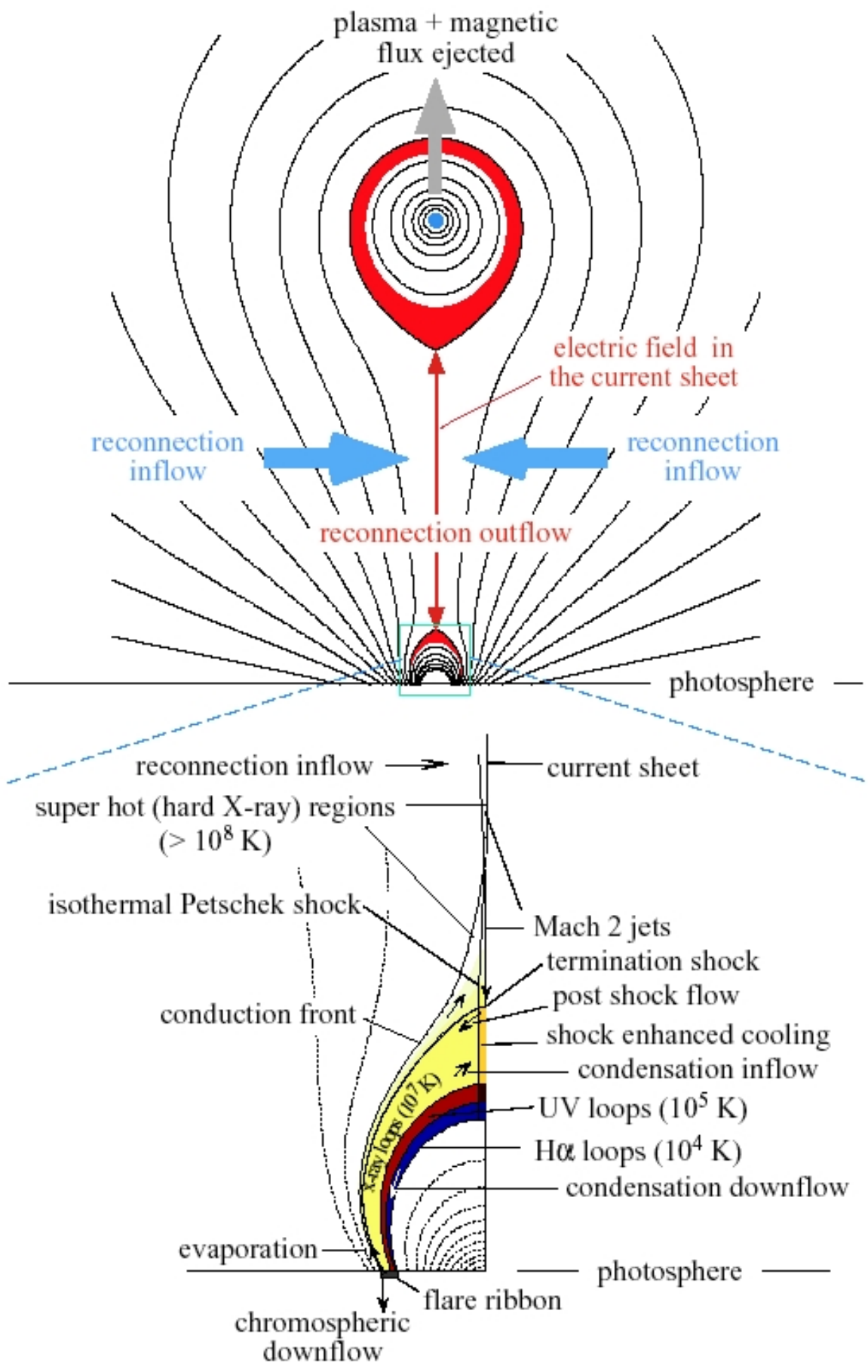

Figure 4.12: Cartoon of a CSHKP model after Lin \& Forbes (2000). 
Coronal mass ejections are frequently associated with filament eruptions. Kuperus \& Raadu (1974) proposed a model, in which cold and dense filaments are supported against gravity due to the induced photospheric currents. This is in agreement with observations, which reveal that the magnetic field of the filaments often exhibits inverse polarity with respect to the photospheric magnetic field Leroy et al. (1983, 1984). The standard CME-flare concept assumes an initial flux-rope topology (Fig. 4.11 a). It is the so-called CSHKP model, named after (Carmichael 1964, Sturrock 1966, Hirayama 1974. Kopp \& Pneuman 1976). An example of a flux-rope model by Lin \& Forbes (2000) is illustrated in Fig. 4.12. In this model, a loss of equilibrium due to flux cancellation occurs, leading to the rise of the flux-rope into a new equilibrium height. According to the classification by Klimchuk (2001) based on mechanical analogies, the CSHKP model belongs to the class of models called "tether release" or "tether cutting". A "tether release" CME initiation model is represented in Fig. 4.13 .

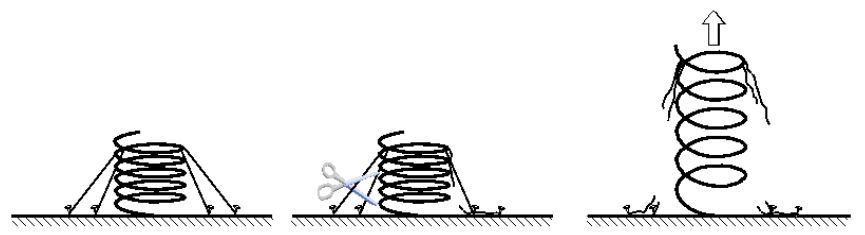

Figure 4.13: Tether release

Non-ideal MHD simulations with an enhanced resistivity at the null point (Forbes 1991, Lin \& Forbes 2000, Amari et al. 2000) have shown, that the loss of equilibrium between the buoyant magnetic pressure and the downward-directed magnetic tension may result in successive cutting of the tethers represented by the magnetic field lines and trigger a CME. Chen et al. (1997) and Dere et al. (1999) have suggested, that the dark cavity observed in a typical three-part CME corresponds to the flux-rope.

\subsubsection{Thermal Blast Model}

A CME driven by a thermal blast pressure wave produced by a flare was the first proposed CME trigger mechanism (Dryer 1982, Wu 1982). In this model, the large energy released in flares results in heating and acceleration of the coronal plasma and an interplanetary shock. Now it is known, that the CMEs are not always associated with flares, and if they are, they often precede the flares, so this model is no longer considered realistic. 


\subsubsection{Dynamo Model}

Stressed magnetic flux builds-up in the corona due to the motion of footpoints of field lines, analogous to the stressing of a spring when an external force is exerted. The stress increase may be due to the twisting of the pre-existing coronal field lines by footpoint motion, or it can be caused by a rise of the newly formed field lines.

\subsubsection{Break-out Model}
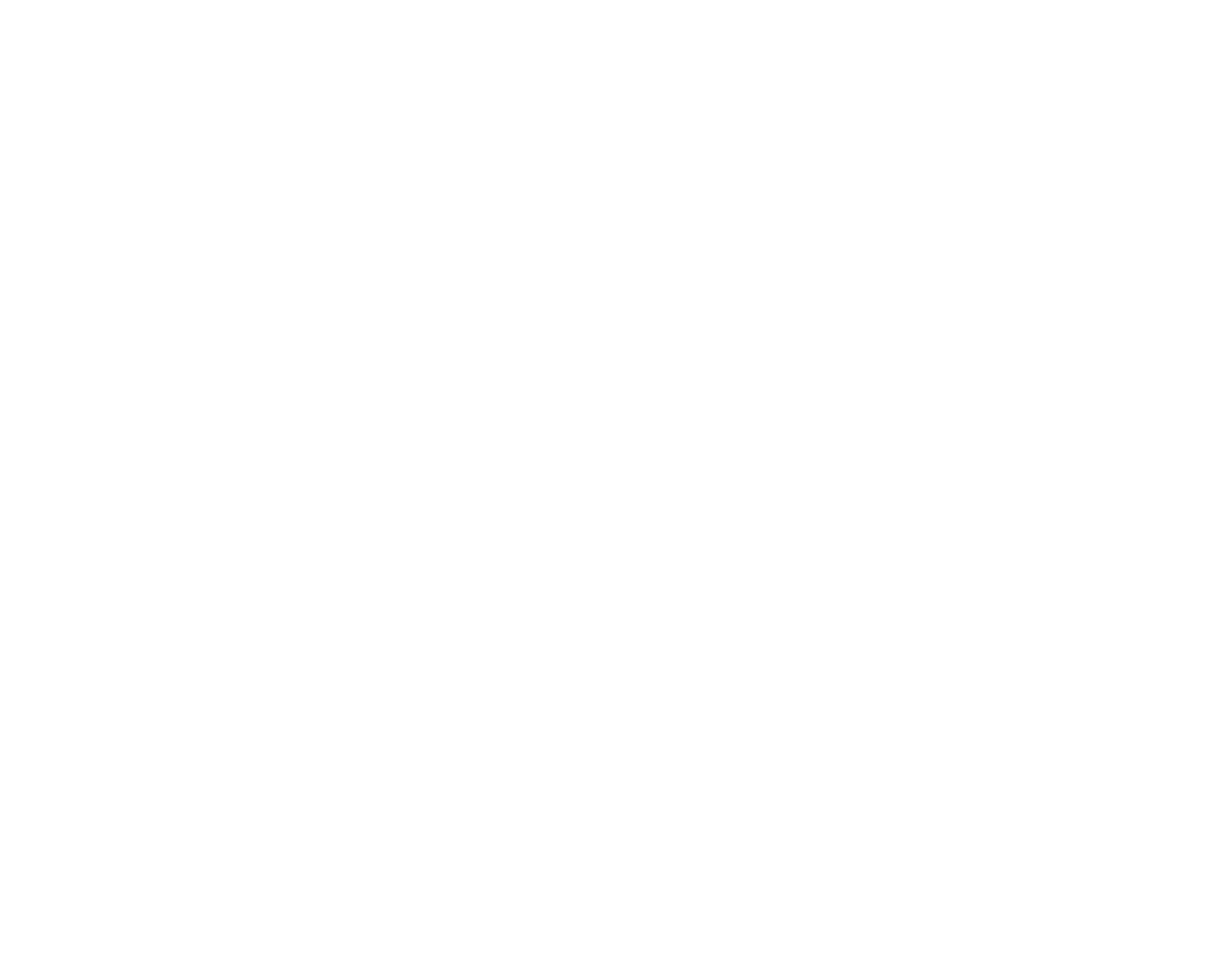

Figure 4.14: Time-dependent solution of the MHD equations for the "Break-out" model (Antiochos et al.1999).

Antiochos et al. (1999) suggested a model for CMEs occurring in multipolar regions with an arcade-like initial topology, the so-called Break-out model. Shearing of the arcade due to the displacement of positive and negative polarities in opposite directions along the neutral line under the effects of differential rotation enables the build up of 
the magnetic free energy. This energy can be released during a flare triggered by the reconnection between the arcade and neighboring flux systems. Gradual increase in
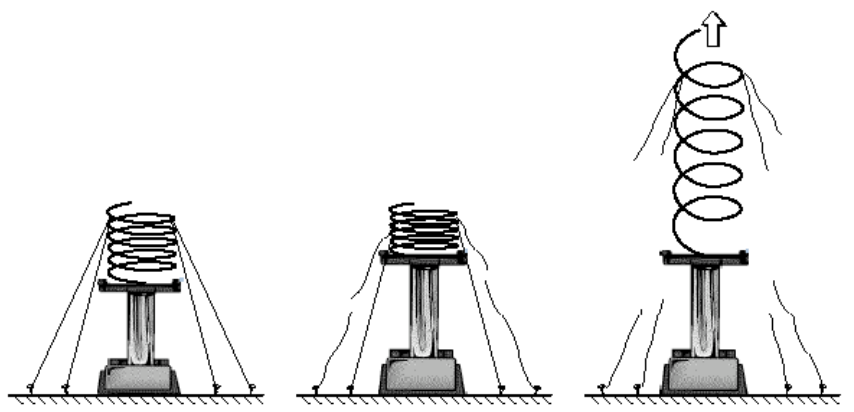

Figure 4.15: Tether straining

magnetic tension of the magnetic field lines which become longer due to buoyant forces results in their break up. It belongs to the "tether straining" (Fig. 4.15) class of models (Klimchuk 2001). Examples of tether-straining models with an initial arcade topology were suggested by Mikic \& Linker (1994), Linker \& Mikic (1995), Choe \& Lee (1996), Amari et al. (1996) . Several other CME observations appear to be explainable in terms of the breakout model (Aulanier et al. 2000, Sterling \& Moore 2001, 2004, Sterling et al. 2001, Wang et al. 2003a, Manoharan \& Kundu 2003, Subramanian et al. 2003, Gary \& Moore 2004), from which the best known is the Bastille flare event (Aulanier et al.2000). 



\section{Coronal Waves and Oscillations}

\subsection{Introduction}

Modern imaging and spectroscopic instruments in the visible, EUV, X-ray and radio bands are able to detect waves and oscillations in the solar corona. Observations of kink, sausage, torsional and longitudinal standing modes as well as the propagating slow and fast waves are interpreted and theoretically modeled in terms of MHD wave theory.

\subsection{Theoretical Overview}

The hot, sparse, magnetically dominated corona is characterized by a low- $\beta$-plasma (Eq. 3.20) and a high Magnetic Reynold's number $R_{m}$ (Eq. 3.22). Magnetic field lines are therefore "frozen into" the plasma and form coronal loops with footpoints anchored into the photosphere.

In case of an equilibrium state (Eq. 4.1), the total pressure inside of the loop must equal the total pressure in the ambient corona.

When the magnetic field lines are pressed together due to an instability, the magnetic tension (4.9) increases. It acts against bending of the loops, as a restoring force to the oppositely directed force due to magnetic pressure (3.4), giving rise to kink (asymmetric) or sausage (symmetric) oscillations. A mechanical analogy of such restoring forces is a string, where the deviation from equilibrium under action of external forces is restored due to the tension of the string. In mechanics, the oscillation period depends on the length of the string and on its tension. The period of MHD waves depends on the magnetic field strength, density and length of the loop.

When a density fluctuation forms in a loop, it is restored by the plasma pressure giving rise to acoustic waves. Compressibility of the medium has the role of elasticity, pressure defined by the temperature acts against it. 


\subsubsection{Definition of Characteristic Phase Speeds of MHD Waves}

MHD waves are the means of information transport in plasma. Five characteristic phase speeds, the speed of sound $c_{s}$, the Alfvén speed $c_{A}$, the tube speed $c_{T}$, the kink speed $c_{k}$ and the fast speed $c_{f}$, are important in the description of the waves.

In the magnetically neutral media, the communication is achieved by sound waves, whose phase velocity in an ideal gas with equilibrium pressure $p_{0}$, density $\rho_{0}$ and adiabatic index $\gamma$, is:

$$
c_{s}=\left(\frac{\gamma p_{0}}{\rho_{0}}\right)^{1 / 2} \text {. }
$$

When magnetic fields are present, the Alfvén speed $c_{A}$ must be introduced:

$$
c_{A}=\left(\frac{B_{0}^{2}}{4 \pi \rho_{0}}\right)^{1 / 2} .
$$

The tube speed, $c_{T}$, is important in descriptions of waves propagating in flux tubes. It is also called the slow speed, because it is both sub-sonic and sub-Alfvénic. It is defined as:

$$
c_{T}^{-2}=c_{s}^{-2}+c_{A}^{-2}
$$

The kink speed, which is a density weighted average Alfvén speed of the medium, is intermediate between the external $c_{\mathrm{A} \text {,ext }}$ and the internal $c_{\mathrm{A} \text {,int }}$ inside the magnetic tube.

$$
c_{k}=\left(\frac{\rho_{\mathrm{int}} c_{\mathrm{A}, \mathrm{int}}^{2}+\rho_{\mathrm{ext}} c_{\mathrm{A}, \mathrm{ext}}^{2}}{\rho_{\mathrm{int}}+\rho_{\mathrm{ext}}}\right)^{1 / 2} .
$$

In the low $\beta$-plasma limit, $c_{k}$ can be approximated as:

$$
c_{k} \approx \sqrt{\frac{2}{1+\rho_{\mathrm{ext}} / \rho_{i}}} c_{A} .
$$

The super-sonic and super-Alfvénic fast speed, $c_{f}$, is defined as:

$$
c_{f}^{2}=c_{s}^{2}+c_{A}^{2}
$$




\subsubsection{MHD Waves in an Unbounded Homogeneous Medium}

By introducing a small localized perturbation in density, velocity, and magnetic induction $\left(\rho_{1}, \mathrm{v}_{1}, B_{1}\right)$ from the equilibrium values $\left(\rho_{0}, \mathbf{v}_{0}, \mathbf{B}_{0}\right)$, while neglecting large-scale background gradients and quadratic and higher order terms, the ideal adiabatic MHD equations (Section 3.3) can be re-written as:

Continuity equation:

$$
\frac{\partial \rho}{\partial t}=-\rho_{0} \boldsymbol{\nabla} \cdot \mathbf{v}_{1}
$$

Momentum equation:

$$
\rho_{0} \frac{\partial \mathbf{v}_{1}}{\partial t}=-c_{s}^{2} \boldsymbol{\nabla} \rho_{1}+\frac{1}{4 \pi}\left[-\boldsymbol{\nabla}\left(\mathbf{B}_{\mathbf{0}} \cdot \mathbf{B}_{\mathbf{1}}\right)+\left(\mathbf{B}_{\mathbf{0}} \cdot \nabla\right) \mathbf{B}_{\mathbf{1}}\right]
$$

Induction equation:

$$
\frac{\partial \mathbf{B}_{\mathbf{1}}}{\partial t}=\nabla \times\left(\mathbf{v}_{1} \times \mathbf{B}_{\mathbf{0}}\right)=\left(\mathbf{B}_{\mathbf{0}} \cdot \nabla\right) \mathbf{v}_{1}-\mathbf{B}_{\mathbf{0}}\left(\boldsymbol{\nabla} \cdot \mathbf{v}_{1}\right) .
$$

For simplicity, the direction of the uniform magnetic field $B_{0}$ is chosen to be in $\mathrm{z}$ direction. Taking the time derivative the momentum equation 5.8, substituting for $\frac{\partial \mathbf{B}_{1}}{\partial t}$ from Eq. 5.9 and for $\frac{\partial \rho_{1}}{\partial t}$ from Eq. 5.7, inserting the sound speed $c_{s}$ and Alfvén speed $c_{A}$, following equation can be obtained:

$$
\frac{\partial^{2} \mathbf{v}_{1}}{\partial t^{2}}=c_{S}^{2} \boldsymbol{\nabla}\left(\boldsymbol{\nabla} \cdot \mathbf{v}_{1}\right)+c_{A}^{2}\left[\frac{\partial}{\partial z}\left(\frac{\partial \mathbf{v}_{1}}{\partial z}-\left(\boldsymbol{\nabla} \cdot \mathbf{v}_{1}\right)_{z}\right)-\boldsymbol{\nabla}\left(\frac{\partial \mathbf{v}_{\mathbf{z}}}{\partial z}-\boldsymbol{\nabla} \cdot \mathbf{v}_{1}\right)\right]
$$

The $z$-component of Eq. 5.10 is:

$$
\frac{\partial^{2} \mathbf{v}_{\mathbf{Z}}}{\partial t^{2}}=c_{S}^{2} \frac{d\left(\boldsymbol{\nabla} \cdot \mathbf{v}_{1}\right)}{d z}
$$

Taking the divergence of Eq. 5.10 yields:

$$
\frac{\partial^{2}\left(\boldsymbol{\nabla} \cdot \mathbf{v}_{1}\right)}{\partial t^{2}}=c_{S}^{2} \boldsymbol{\nabla}^{2}\left(\boldsymbol{\nabla} \cdot \mathbf{v}_{1}\right)+c_{A}^{2} \boldsymbol{\nabla}^{2}\left(\boldsymbol{\nabla} \cdot \mathbf{v}_{1}-\frac{\partial \mathbf{v}_{\mathbf{z}}}{\partial z}\right)
$$

If $\boldsymbol{\nabla} \cdot \mathbf{v}_{1}=0$ is inserted into Eq. 5.12, the $z$-component of the velocity vanishes $\left(\mathrm{v}_{\mathrm{z}}=0\right)$ and Eq. 5.10 can be re-written as:

$$
\frac{\partial^{2} \mathbf{v}_{1}}{\partial t^{2}}=c_{A}^{2} \frac{\partial^{2} \mathbf{v}_{1}}{\partial z^{2}},
$$


which corresponds to the equation for pure Alfvén waves which are incompressible $\left(\boldsymbol{\nabla} \cdot \mathbf{v}_{1}=0\right)$, and propagate along the magnetic field $\mathbf{B}_{\mathbf{0}}$.

If $\boldsymbol{\nabla} \cdot \mathbf{v}_{1} \neq 0, \mathbf{v}_{\mathrm{z}}$ can be eliminated from the system of equations 5.11 and 5.12 and a the following equation can be derived:

$$
\frac{\partial^{4}\left(\boldsymbol{\nabla} \cdot \mathbf{v}_{1}\right)}{\partial t^{4}}-\left(c_{A}^{2}+c_{S}^{2}\right) \frac{\partial^{2}}{\partial t^{2}}\left[\boldsymbol{\nabla}^{2}\left(\boldsymbol{\nabla} \cdot \mathbf{v}_{1}\right)\right]+c_{A}^{2} c_{S}^{2} \frac{\partial^{2}}{\partial z^{2}}\left[\boldsymbol{\nabla}^{2}\left(\boldsymbol{\nabla} \cdot \mathbf{v}_{1}\right)\right]
$$

Expressing the perturbed quantities in Fourier form $\propto \exp [i \omega t+i \mathbf{k} \cdot \mathbf{r}]$ leads to the substitutions $\partial / \partial t=i \omega^{2}$ and $\boldsymbol{\nabla} \cdot=i \mathbf{k}$, so the dispersion relation for possible MHD modes is (Cowling 1976, Roberts 1981):

$$
\mathrm{v}_{\mathrm{ph}}^{4}-\mathrm{v}_{\mathrm{ph}}^{2}\left(c_{A}^{2}+c_{s}^{2}\right)+c_{A}^{2} c_{s}^{2} \cos ^{2} \Theta=0,
$$

where $\Theta$ is the angle between the magnetic field $\mathbf{B}_{0}$ and $\mathbf{k}$. The phase speed diagram $\mathrm{v}_{\mathrm{ph}}(\Theta)$ for different ratios of $c_{A} / c_{s}$ is shown in Fig. 5.1.

\subsubsection{Special solutions}

1. In the absence of magnetic field, $c_{A}=0$, the dispersion equation 5.15 reduces to $\mathrm{v}_{\mathrm{ph}}=\mathrm{c}_{\mathrm{s}}$, a solution of a pure acoustic mode. The dependence on the direction of propagation vanished, the acoustic mode is therefore non-dispersive: $\omega / k=$ $d \omega / d k=c_{s}$. Inserting the Fourier form of the perturbation $\propto \exp [i \omega t+i \mathbf{k} \cdot \mathbf{r}]$ into the continuity equation 5.7 yields

$$
c_{s} \rho_{1}=\mathrm{v}_{1} \rho_{0},
$$

which says, that the perturbation $\mathrm{v}_{1}$ is restored by a proportional change in density.

2. For the wave propagation perpendicular to the magnetic field, $\Theta=90^{\circ}$, the third addend in the dispersion equation 5.15 vanishes as well and the solution becomes:

$$
\mathrm{v}_{\mathrm{ph}}=\sqrt{c_{s}^{2}+c_{A}^{2}}
$$

3. For the wave propagation parallel to the magnetic field, $\Theta=0^{\circ}$, there are 2 solutions:

$$
v_{p h 1,2}^{2}=\frac{\left(c_{s}^{2}+c_{A}^{2}\right) \pm\left(c_{s}^{2}-c_{A}^{2}\right)}{2}=\left\{\begin{array}{l}
c_{S}^{2} \\
c_{A}^{2}
\end{array}\right.
$$



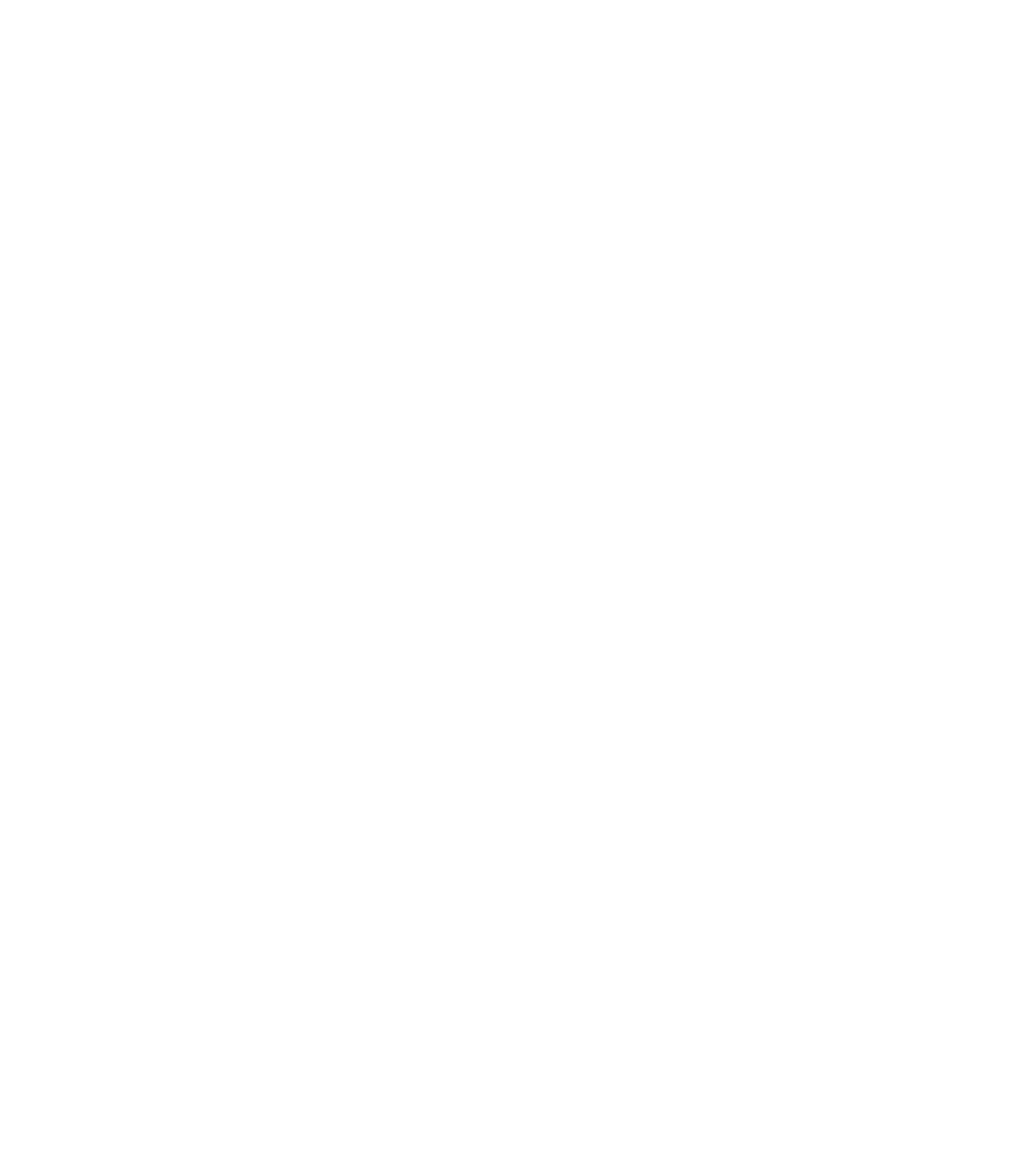

Figure 5.1: Friedrichs diagram (animation by Volker Gaibler) representing the superAlfvénic or fast (red), Alfvén or intermediate (black), and slow (blue) phase velocities in units of adiabatic speed of sound as a function of the direction with respect to the direction of the magnetic induction (arrow) for Alfvén velocities from 0 to 3 times the speed of sound.

\subsubsection{General solutions}

For every propagation angle $\Theta$, there are generally three solutions of the dispersion equation 5.15: slow, intermediate, or fast magneto-acoustic mode. They are represented 
in the Fig. 5.1 .

\section{Alfvén wave - intermediate mode}

Magnetohydrodynamic plasma is a medium, which can support MHD waves propagating without compressing the plasma. Shear Alfvén waves are transversal and incompressible $\left(\mathbf{k v}_{1}=0\right.$ and $\left.\boldsymbol{\nabla} \cdot \mathbf{v}_{1}=0\right)$. After inserting $\boldsymbol{\nabla} \cdot \mathbf{v}_{1}=0$ into the induction equation 5.9 it follows, that $\left.\omega \mathbf{B}_{1}=\left(\mathbf{k} \mathbf{B}_{\mathbf{0}}\right) \mathbf{v}_{1}\right)$, i.e. the only restoring force for the velocity perturbations $\mathbf{v}_{1}$ are the changes in magnetic flux density. The general solution of shear Alfvén wave is:

$$
\omega / k=\mathrm{v}_{\mathrm{ph}}=\mathrm{c}_{\mathrm{A}} \cdot \cos \Theta,
$$

with Alfvén speed (5.2) as a special solution for $\Theta=0^{\circ}$. When propagating parallel to the magnetic field, the shear Alfvén wave has a maximum speed $c_{A}$. It cannot propagate in the perpendicular direction $\Theta=90^{\circ}$ (see the polar diagram 5.1.

\section{Slow Acoustic Mode}

Slow acoustic waves are compressible and longitudinal, with phase speed in the range $c_{T}<\mathrm{v}_{\mathrm{p}} \mathrm{h}<\min \left(c_{s}, c_{A}\right)$ with a maximum propagation speed along the magnetic field. They cannot propagate perpendicularly to the magnetic field.

\section{Fast Acoustic Mode}

The phase speed of the fast mode ranges from $\max \left(c_{s}, c_{A}\right)<\mathrm{v}_{\mathrm{ph}}<\left(c_{s}^{2}+c_{A}^{2}\right)^{1 / 2}$, and it reaches maximum for propagation perpendicular to the magnetic field.

\subsubsection{Waves in a Straight Magnetic Cylinder}

The solar atmosphere is a highly inhomogeneous medium. Owing to the presence of boundaries formed by magnetic structures, surface waves occur. In order to derive the possible MHD modes to be found in the magnetic loops, protuberances and polar plumes, the waves in magnetic cylinder (Fig. (5.2) need to be explored. 


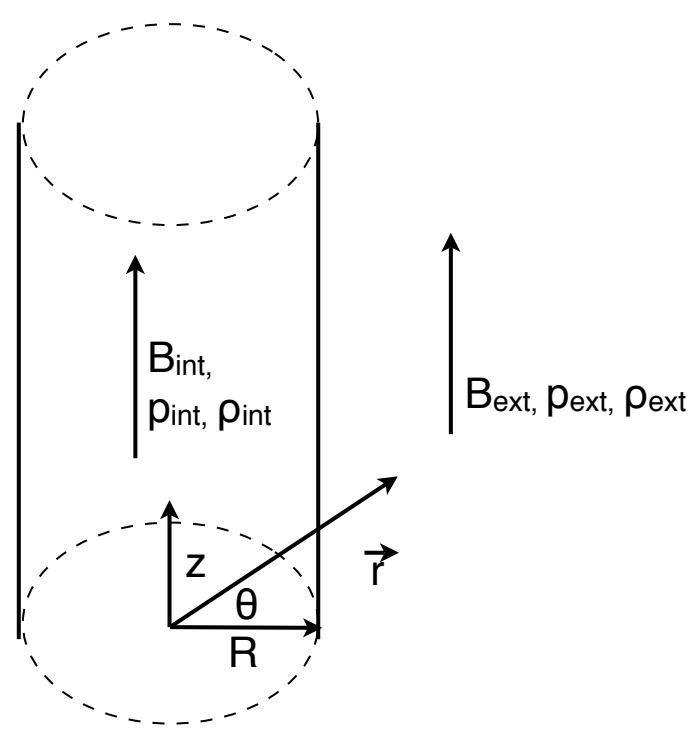

Figure 5.2: Representation of a magnetic tube with a radius $R$.

The three basic MHD waves, fast, slow and intermediate, described in the Section 5.2.2, propagate in a straight magnetic cylinder. The properties of MHD waves strongly depend on the angle between the direction of propagation and the magnetic field (Fig. 5.1 ). Hence, magnetic structures present in the solar corona give rise to mode coupling, phase mixing, resonant absorption, and guided wave propagation.

Linear perturbations about the pressure equilibrium given by 4.1 lead to the wave equations (Roberts 1981):

$$
\frac{\partial^{2}}{\partial t^{2}}\left(\frac{\partial^{2}}{\partial t^{2}}-\left(c_{S, \text { int }}^{2}+c_{\mathrm{A}, \text { int }}^{2}\right) \nabla^{2}\right) \boldsymbol{\nabla} \cdot \mathbf{v}_{1}+c_{S, \text { int }}^{2} c_{\mathrm{A}, \text { int }}^{2} \frac{\partial^{2}}{\partial z^{2}} \nabla^{2}\left(\boldsymbol{\nabla} \cdot \mathbf{v}_{1}\right)=0
$$

and

$$
\left(\frac{\partial^{2}}{\partial t^{2}}-c_{\mathrm{A}, \mathrm{int}}^{2} \frac{\partial^{2}}{\partial z^{2}}\right)\left(\boldsymbol{\nabla} \times \mathbf{v}_{1}\right)_{z}=0
$$

inside the magnetic cylinder. Same equations are valid for $c_{S, \text { ext }}^{2}, c_{\mathrm{A}, \text { ext }}^{2}$ outside the the cylinder. If we write the Fourier form of $\boldsymbol{\nabla} \cdot \mathbf{v}_{1}=\mathcal{B}(\mathbf{r}) \exp \left\{i\left(\omega t+n_{H} \Theta+k_{z} z\right)\right\}$, then the function $\mathcal{B}(r)$ must satisfy the Bessel equation

$$
\frac{d^{2} \mathcal{B}}{d r^{2}}-\frac{1}{r} \frac{d^{2} \mathcal{B}}{d r}-\left(\mathcal{M}^{2}+\frac{n_{H}^{2}}{r^{2}}\right) \mathcal{B}=0,
$$




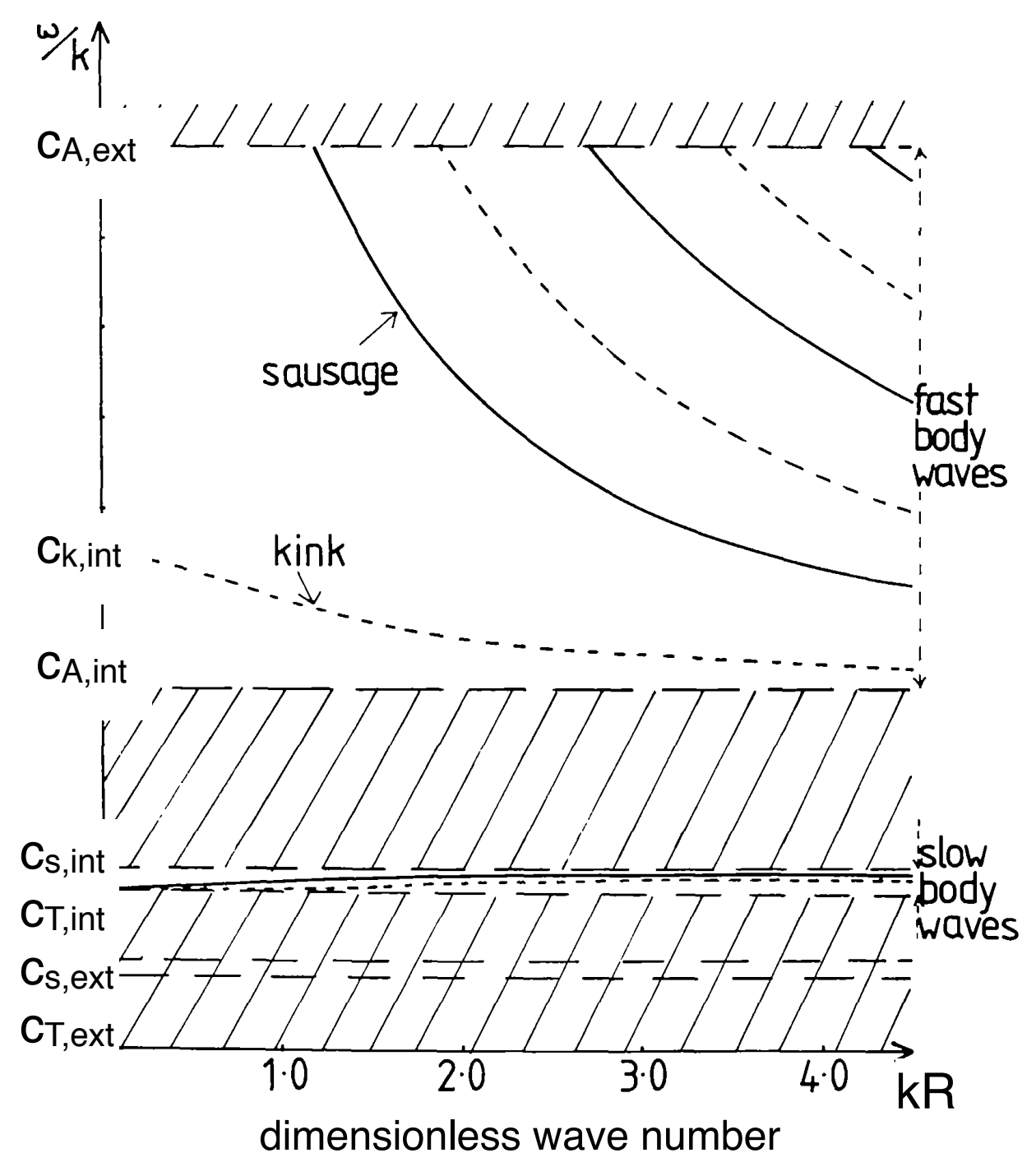

Figure 5.3: Phase-speed diagram representing the spectrum of surface and body waves in a magnetically structured corona for coronal conditions: $c_{s e}<c_{s}<c_{A}<c_{\mathrm{A} \text {,ext }}$.

where $\mathcal{M}$ is the transverse wave number defined in each medium as:

$$
\mathcal{M}^{2}(\omega)=-\frac{\left(\omega^{2}-c_{s}{ }^{2} k_{z}^{2}\right)\left(\omega^{2}-c_{A}{ }^{2} k_{z}{ }^{2}\right)}{\left(c_{s}^{2}+c_{A}{ }^{2}\right)\left(\omega^{2}-c_{T}{ }^{2} k_{z}^{2}\right)},
$$

Applying the boundary conditions to the solutions of the Bessel equation (5.22) leads to the dispersion relation for magneto-acoustic waves in a magnetic flux tube (McKenzie 
1970, Spruit 1982, Edwin \& Roberts 1983):

$$
\rho_{\mathrm{ext}}\left(\omega^{2}-c_{\mathrm{A}, \mathrm{ext}}^{2} k_{z}^{2}\right) m_{\mathrm{int}} \frac{I_{n}^{\prime}\left(m_{\mathrm{int}} R\right)}{I_{n}\left(m_{\mathrm{int}} R\right)}-\rho_{\mathrm{int}}\left(\omega^{2}-c_{\mathrm{A}, \mathrm{int}}{ }^{2} k_{z}^{2}\right) m_{\mathrm{ext}} \frac{K_{n}^{\prime}\left(m_{\mathrm{ext}} R\right)}{K_{n}\left(m_{\mathrm{ext}} R\right)}=0
$$

where $R$ is the radius of the magnetic cylinder (Fig. 5.2), $I_{n}$ and $K_{n}$ are modified Bessel functions of order $n_{H}$, the prime denotes the derivativation with respect to the argument in the brackets and subscripts $e$ and $i$ refer to external and internal media respectively. Body modes $\left(m^{2}(\omega)<0\right)$ are purely acoustic and longitudinal, surface modes $\left(m^{2}(\omega)>\right.$ $0)$ are purely magnetic and transversal.

\subsubsection{Alfvén Waves}

Pure Alfvén waves in flux tubes manifest as torsional oscillations of the loops (Erdélyi \& Fedun 2007). Torsional modes of untwisted coronal loops are described by in the Eq. 5.21. They are incompressible and, therefore, do not perturb the density, and cannot be seen as intensity fluctuations. If a twist is introduced, density is perturbed and intensity oscillations would be also present. Alfvén waves create simultaneous blue and red shifts, and if the spatial resolution of the spectrometer is less than the width of the observed loop, they can be observed as Doppler width oscillations. The maximum amplitude would be observed at the antinodes and minimum at the nodes.

\subsubsection{Sausage Mode and Kink Mode}

Solving the equation $(5.22)$ in the incompressible limit $\left(c_{s} \rightarrow 0\right)$ and $m\left(\omega_{e, i}\right) \rightarrow|k|$, gives a dispersion relation:

$$
\omega^{2}=k^{2} \frac{c_{\mathrm{A}, \mathrm{ext}}^{2}-\left(\rho_{\mathrm{int}} / \rho_{\mathrm{ext}}\right) c_{\mathrm{A}, \mathrm{ext}}{ }^{2} \phi_{n}}{1-\left(\rho_{\mathrm{int}} / \rho_{\mathrm{ext}}\right) \phi_{n}},
$$

where $\phi_{n}=I_{n}(a|k|) K_{n}^{\prime}(a|k|) / I_{n}^{\prime}(a|k|) K_{n}(a|k|)$. The characteristic phase speeds $\omega / k$ can be calculated and represented in the dispersion diagram (5.3). In the solar corona, the symmetric sausage mode $(\mathcal{M}=0)$, and the antisymmetric kink mode $(\mathcal{M}=1)$ are important. The phase velocity of the sausage mode approaches the external Alfvén speed (Eq. 5.2). The kink speed $c_{k}$ is defined in Eq. 5.4 and its approximation in the limit of low $\beta$-plasma is given in Eq. 5.5. Kink oscillations manifest as quasi-periodic transverse displacements of the loops. The horizontal kink doesn't vary the loop length and is therefore incompressible. In contrast, the vertical kink mode in a curved loop may have a significant compressible component and can be seen as intensity fluctuations (Wang \& Solanki 2004). 


\subsubsection{Slow Magneto-acoustic Waves}

The slow magneto-acoustic waves are longitudinal, perturbing the density and the component of velocity parallel to the energy propagation and to the magnetic field. Since the emissivity of EUV lines roughly scales as the square of the density, assuming the magnetic field has a component parallel to the line-of-sight, they may be observed as periodic variations of the intensity and Doppler shift. In the closed magnetic structures, such as loops, standing waves (oscillations) form, due to the reflecting of the wave from the dense atmosphere at the footpoints. Neglecting energy dissipation and the magnetic field the continuity (5.7) and momentum (5.8) equations become:

$$
\frac{\partial \rho_{1}}{\partial t}=-\rho_{0} \boldsymbol{\nabla} \cdot \mathbf{v}_{1}
$$

and

$$
\rho_{0} \frac{\partial \mathbf{v}_{1}}{\partial t}=-c_{s}^{2} \nabla \rho_{1}
$$

which, combined together, give an equation of a harmonic oscillator:

$$
\frac{\partial^{2} \mathbf{v}_{1}}{\partial t^{2}}=-c_{2}^{2} \rho_{0} \boldsymbol{\nabla}\left(\partial \rho_{1} / \partial t\right)=c_{s}^{2} \nabla^{2} \mathbf{v}_{1},
$$

whose general solution is

$$
\begin{gathered}
\mathrm{v}(\mathrm{z}, \mathrm{t})=A \cos \left(\frac{\mathrm{n}_{\mathrm{H}} \pi}{2 \mathrm{~L}} \mathrm{z}\right) \sin \left(\frac{\mathrm{n}_{\mathrm{H}} \pi \mathrm{c}_{\mathrm{s}}}{2 \mathrm{~L}} \mathrm{t}\right) \\
\rho(z, t)=-\frac{A \rho_{0}}{c_{s}} \sin \left(\frac{n_{H} \pi}{2 L} z\right) \cos \left(\frac{n_{H} \pi c_{s}}{2 L} t\right),
\end{gathered}
$$

The phase shift between velocity and density perturbations is $-\pi / 2$ and 0 for standing and propagating waves respectively. The observed phase shift may differ from the theoretically predicted one when the variations of the background density and temperature become important. Density gradients at the footpoints anchored into the transition region are also adding into this affect by not providing a perfect reflecting boundary for the formation of standing sound waves.

Numerical simulations conducted by (Tsiklauri et al.2004) revealed the second harmonic of a standing wave with a velocity oscillation node and the density oscillation maximum at the loop apex, to be the dominant mode, independently of the varying characteristics of the flare (duration, peak average temperature, etc.). At first, they attributed this trend to the symmetric nature of the excitation of the oscillations due to the 
heat deposition near the loop apex. Though, breaking the symmetry by moving the heat deposition near one of the footpoints again showed the second harmonic as the most significant mode. In comparison with the case of symmetric heating, the position of the velocity node of the oscillation at the apex of the loop wasn't constant, but it oscillated along the apex. Strong flows present in the asymmetric case may vary the background emission along the line-of-sight significantly, making the interpretation of the modes difficult.

SUMER spectrograph has discovered strongly damped quasi-periodic oscillations of the Doppler shift of the flare lines Fe XIx and Fe xxI Kliem et al. (2002), Wang et al. (2002). Although the SUMER slit records in EUV part of the spectra covering temperatures from 0.01 to $10 \mathrm{MK}$, Doppler shift oscillations have only been detected in lines with the formation temperature above $1 \mathrm{MK}$.

\subsection{Non-Ideal MHD Effects}

In equations (3.1 - 3.11), non-ideal effects such as resistivity, viscosity, and Ohmic dissipation, which might contribute to damping of the waves have been neglected. Furthermore, the curvature and twist of loops hasn't been considered in the derivation of the dispersion relation of the waves (5.24). According to (Zhugzhda \& Nakariakov 1999), the appearance of the dispersion (as the slab has a finite width) of torsional waves in a twisted flux tube generate longitudinal flows, and consequently density perturbations. By using the adiabatic equation of state, it is assumed that the heating, thermal conduction, and radiative loss are neglected in the energy equation. 



\section{Multi-wavelength Observations of Oscillations in the Wake of a Flare Blast Wave}

Oscillations in flare loops are caused by a rapid injection of energy to the plasma in the loop. Kink oscillations, manifested as periodic transverse displacements of coronal loops, are believed to be triggered by a flare blast wave hitting the loop edge-on (Aschwanden et al. 1999, 2002, Nakariakov et al.|1999, Schrijver et al.|2002). In their study, Hudson \& Warmuth (2004) found 12 out of 28 cases of TRACE oscillations coinciding with Type II bursts, supporting their strong connection with large-scale flare shocks.

\subsection{Instruments}

\subsubsection{Solar Ultraviolet Measurements of Emitted Radiation (SUMER) on board of Solar Heliospheric Observatory (SoHO)}

ESA/NASA jointly-built the Solar and Heliospheric Observatory (SoHO) spacecraft, launched in 1995. It slowly orbits around the first Lagrangian point situated around 1.5 million kilometers away from Earth towards the Sun, and it allowed continuous observations of he Sun for the first time.

Spectroscopic observations of the inner solar corona are performed by the SUMER (Solar Ultraviolet Measurements of Emitted Radiation) telescope and spectrometer designed and built at the Max Planck Institute for Solar System Research (Wilhelm et al. 1995). It contains 4 entrance slits $(1$ " $\times 300$ " , 1 " $\times 120$ " , 0.3 ” $\times 120$ ', 4 " $\times 300$ ” ) returning 1-dimensional spectral observations in either raster or sit-and-stare mode. Using spectroscopic diagnostics of the chromospheric and coronal emission lines from $330 \AA-1610 \AA$ covering a wide temperature range from $10^{4}-2 \times 10^{6} \mathrm{~K}$, the SUMER spectrometer allows us to study dynamical processes taking place in the atmosphere of the Sun as well as to estimate plasma parameters such as electron temperature and number density. The size of a spectral pixel is around $44 \mathrm{~m} \AA$ and $22 \mathrm{~m} \AA$ in first and second 
order of the detector respectively decreasing for longer wavelengths. The spatial angular size of a pixel is around $1 "$ and minimum sampling of $\approx 3-10 \mathrm{~s}$ can be achieved. Such high spectral, spatial and temporal resolution provide us with high accuracy of the measurements of Doppler velocities down to $1 \mathrm{~km} \mathrm{~s}^{-1}$ for each spatial pixel.

\subsubsection{Transition Region And Coronal Explorer (TRACE)}

TRACE (Handy et al. 1999) imaging observations of the transition region and corona have a high spatial ( $\approx 1$ ") and temporal (less than min) resolution. A 30-cm Cassegrain telescope with a field of view of $(8,5 \times 8,5$ arc minutes $)$ operates in three coronal EUV bands ( Fe IX / Fe x $171 \AA$; Fe XII /Fe xxIV $195 \AA$ and Fe XV $284 \AA$ ), as well as in $\mathrm{H}$ I Lyman $\alpha(1216 \AA)$, C IV (1550 ̊), UV continuum (1600-1700 ̊), and white light (5000 $\AA$ ) covering the temperatures from $6000 \mathrm{~K}$ to $1 \mathrm{MK}$, with the main sensitivity in the 1-2 MK range for the EUV filters.

\subsubsection{Meudon Heliograph}

The Meudon heliograph with a Lyot filter observes the full Sun in three bands: in the core of the $\mathrm{H} \alpha$ line at $6563 \AA$ and in the wings located $0.5 \AA$ from the center.

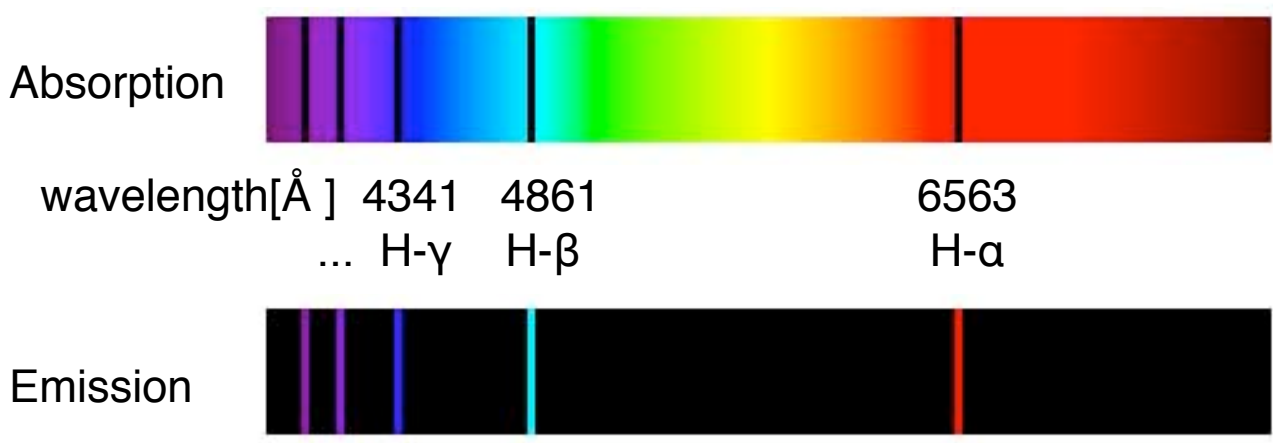

Figure 6.1: Emission and absorbtion of the Hydrogen. Spectral lines named $\mathrm{H} \alpha,-\beta,-\gamma$, $-\delta,-\ldots$ result from the transitions $3 \rightarrow 2,4 \rightarrow 2,5 \rightarrow 2,6 \rightarrow 2, \ldots$, resp.

The cadence is around 1 per minute and the size of a spatial pixel is $1.95 "$. Red visible $\mathrm{H} \alpha 6562,8 \AA$ line forms at around $T \sim 3 \times 10^{4} \mathrm{~K}$. It is the strongest line from the Balmer series of Hydrogen (Fig. 6.1), which are due to the cascading from the higher energy levels $(n>2)$ to the state $(n=2)$. It involves the transition from the quantum state $n=3$ to $n=2$. 


\subsection{Wavelet Analysis}
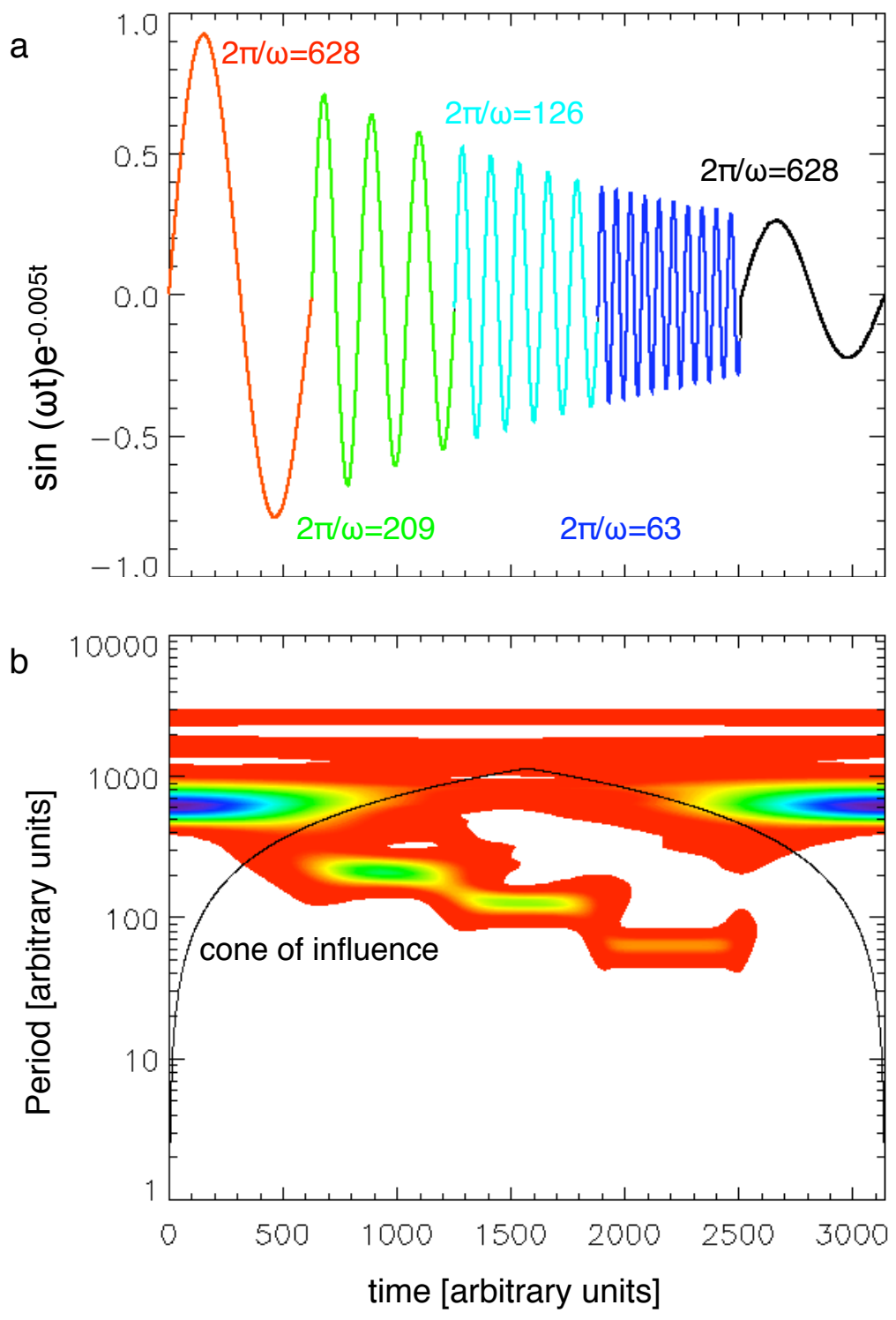

Figure 6.2: a) A damped oscillation with four different oscillation periods localized in five temporal windows. The periods of the signal within the five intervals are in arbitrary units.

b) Representation of the above signal into time-frequency domain. The colors represent the wavelet power (blue is the strongest, then green, yellow, red and white) calculated as the modulus of the wavelet coefficients as a function of time and scale. The cone of influence represents the maximum significant period at a given time for which the edge effects are not important. 
Wavelet analysis is a powerful tool in image denoising and in time series analysis. A signal represented as a function $\mathcal{S}(\mathcal{T})$ defined in the time domain can be transformed into the time-frequency domain (Fig. 6.2).

When used as an image enhancement tool, the pixel saturation which is a (2-dimensional) function of position can be decomposed into different scales and then reconstructed according to a suitable scheme.

\subsubsection{Time Series Analysis}

Rather than applying the scale-dependent Fourier Transform (A.6), a wavelet-based method of time-frequency localization is often applied in analysis of time series characterized by a wide range of dominant frequencies. Wavelet analysis involves transform of 1-dimensional time-series into a 2-dimensional image, which is a function of time and frequency. Decomposition of a time series with non-stationary power into timefrequency space allows to determine both the dominant frequencies of the given variability and its evolution with time.

In Fig. 6.2 a, a damped multi-frequency signal is represented. Below (Fig. 6.2p), the wavelet spectrum of the signal is plotted. The strongest power (blue color) corresponds to the highest modulus of the wavelet coefficients (defined in 6.3) at a given scale and time. The cone of influence is the region of the wavelet spectrum in which edge effects become important. At each scale, it is defined as the e-folding time for the autocorrelation of the wavelet power. In our analysis, Morlet wavelet (Eq. 6.1p has been used, for which the e-folding time is $\sqrt{2} s$.

\subsubsection{Continuous Wavelet Transform}

Oscillatory behavior of a signal can be studied using a continuous wavelet transform which is derived from the windowed Fourier transform (A.19). The latter is used in a non-stationary analysis, as it assumes stationary behavior in the given temporal window. Gabor (1946) introduced a transform windowed by a Gaussian window $\mathcal{G}\left(\mathcal{T}^{\prime}-\mathcal{T}\right)$ (Eq. A.20) sliding in the time domain, thus forming a basis of windowed sinusoids. It corresponds to the convolution with the conjugate of the Morlet wavelet (Eq. 6.1 and Fig. 6.3).

The continuous wavelet transform (CWT) is a generalized Gabor's transform. It involves a convolution with the scaled conjugates of a mother wavelet function $\Psi^{*}$, which is equivalent to the filtering of the signal. 


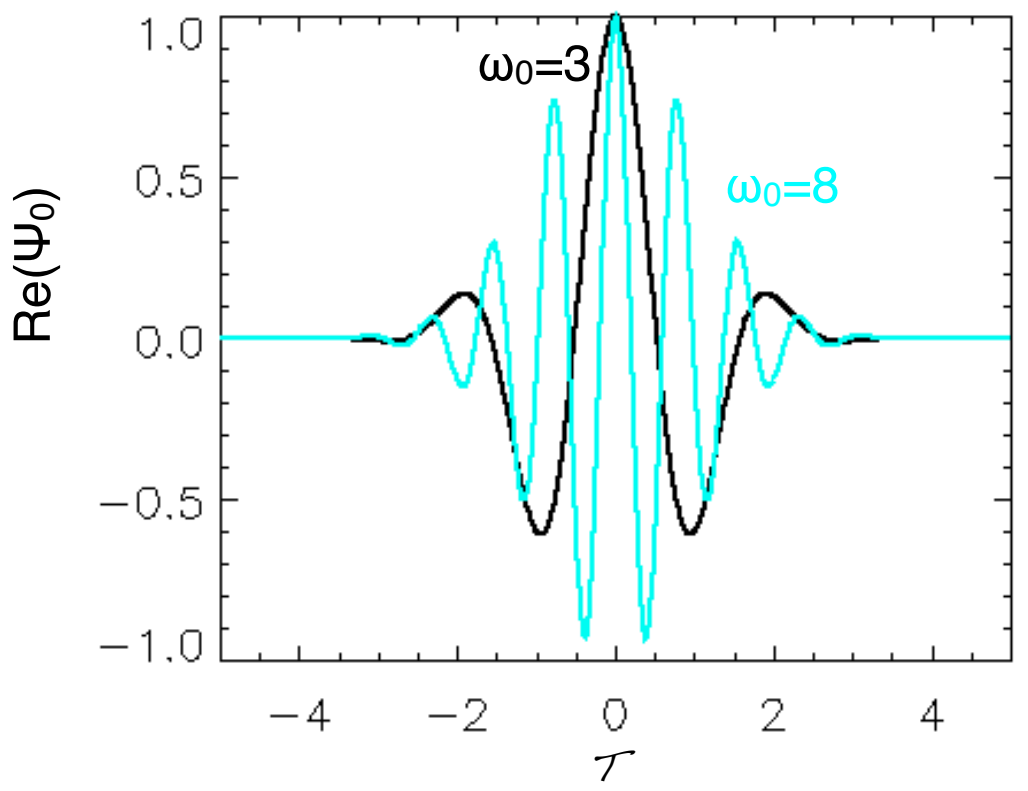

Figure 6.3: Real part of the Morlet wavelet function for 2 different frequencies as a function of dimensionless time $\mathcal{T}$.

A suitable mother wavelet for the analysis of oscillations is a complex wavelet formulated by Morlet:

$$
\Psi_{0}(\mathcal{T})=\pi^{-1 / 4} e^{i \omega_{0} \mathcal{T}} e^{-\mathcal{T}^{2} / 2}
$$

Its real part for two different frequencies $\omega_{0}$ is illustrated in Fig. 6.3. It's conjugate is proportional to the Gabor's transform (Eq. A.19).

A scaled, normalized and translated daughter wavelet is

$$
\frac{1}{\sqrt{s}} \Psi\left(\frac{\mathcal{T}-\mathcal{T}^{\prime}}{s}\right)
$$

It is the function $\Psi_{0}$ translated in time by $\mathcal{T}^{\prime}$ with a modified frequency $s \cdot \omega$. The normalization is necessary in order to be able to compare significance of various frequencies present in the signal. $s$ is the scale parameter related to the frequency, which defines the width of the filter. $\mathcal{T}^{\prime}$ is the position parameter related to the time in the time series analysis (it is related to the position in the image processing). A summation is used instead of an integration because the observations are given by a discrete series $\mathcal{S}\{\mathcal{T}\}$. 
According to the convolution theorem A.21), a wavelet coefficient of a time series $\mathcal{S}(\mathcal{T})$ with a uniform cadence $\Delta t$ at a given scale $s$ and position in time $\mathcal{T}$ can be written as:

$$
\mathcal{W}(s, \mathcal{T})=\sum_{\mathcal{T}^{\prime}=0}^{N-1} \hat{\mathcal{S}}_{\mathcal{T}^{\prime}}, \hat{\Psi}^{*}\left(s \omega_{\mathcal{T}^{\prime}}\right) e^{i \omega_{\mathcal{T}}, \mathcal{T} \Delta t},
$$

where $\hat{\mathcal{S}}_{\mathcal{T}}$, and $\hat{\Psi}^{*}\left(\omega_{\mathcal{T}}\right)$ are the Fourier transforms of the original time series and of the complex conjugate of the wavelet function.

The frequency $\omega_{\mathcal{T}}$ is defined as:

$$
\omega_{\mathcal{T}}= \begin{cases}+2 \pi \mathcal{T} /(N \Delta t) & \text { for } \mathcal{T} \leq N / 2 \\ -2 \pi \mathcal{T} /(N \Delta t) & \text { otherwise }\end{cases}
$$

The wavelet power $\left|\mathcal{W}(s, \mathcal{T})^{2}\right|$ gives an information on the significance of present frequencies. In the analysis of the oscillations presented in the Sections 6.3 and 6.4 the routine /usr/local/ssw/soho/sumer/idl/contrib/safari/package/wavelet.pro has been used in order to find the dominant periods. The output of the routine is a complex two-dimensional array which contains the wavelet coefficients $\mathcal{W}(s, \mathcal{T})$ of a onedimensional time series. The power maps of the oscillating regions are plotted in Fig. 6.11).

\subsubsection{Image Enhancement - Unsharp Masking}

Simultaneous high resolution EUV imaging and spectroscopic observations of flares are very rare. If the contrast of the obtained observations is not high enough, an edge enhancing method is often useful.

The enhancement of the TRACE images used in the analysis of the flare events in Fig. 6.7 was achieved with a method called "unsharp masking" (Malin 1977). It is method based on a two dimensional a trous algorithm. In the presented work, we have used a routine, which involves adding the high-frequency components of the data multiple times. 


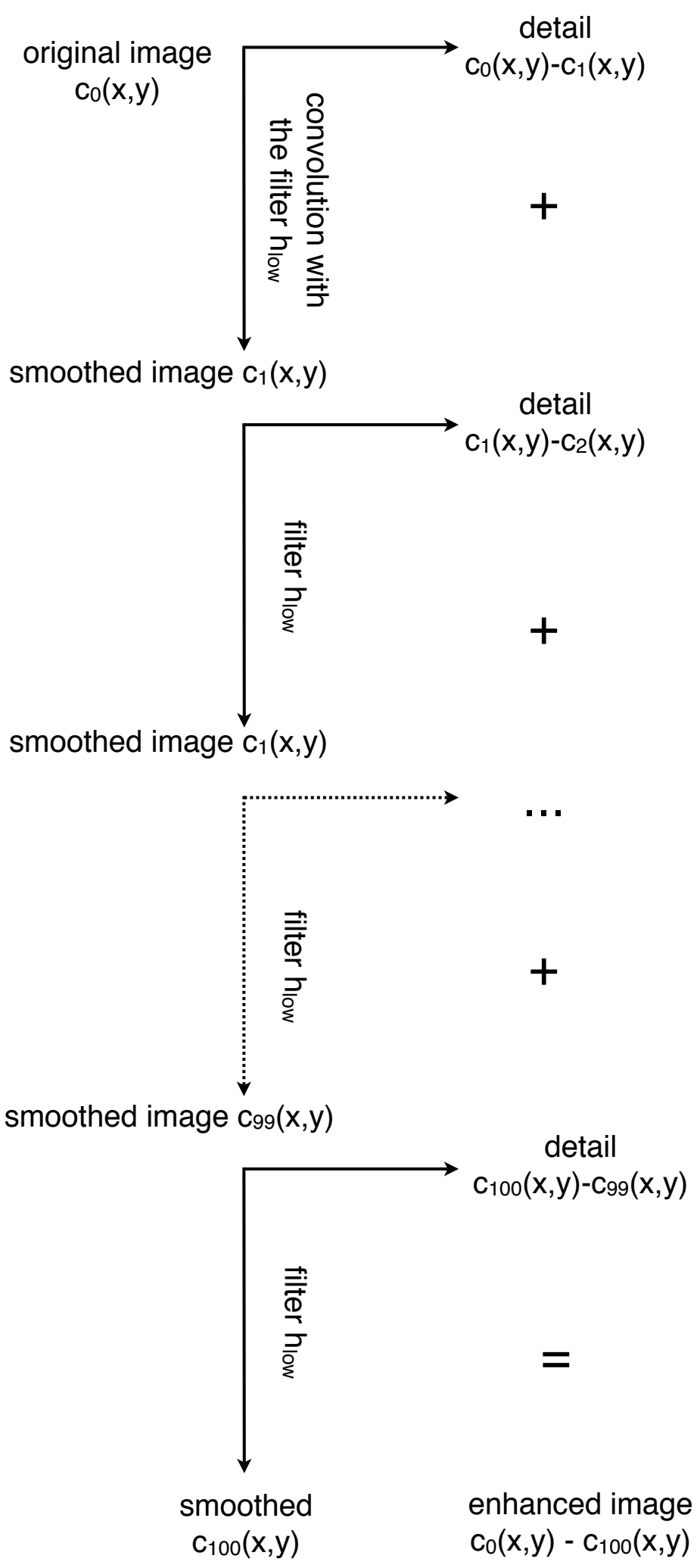

Figure 6.4: Unsharp masking algorithm scheme. 
Decomposition and reconstruction of the image used in our analysis (Fig. 6.4) is described here:

- The original image can be described as 2 dimensional series $c_{0}(x, y)$. The parameters $x, y$ are related to the horizontal and vertical position on the image.

- First, the data are smoothed with a convolution with the $B_{3}$ spline wavelet for scaling function chosen by Stenborg \& Cobelli (2003):

$$
c_{1}(x, y)=\sum_{l, m} h_{\mathrm{low}}(l, m) c_{0}\left(x+2^{0} l, y+2^{0} m\right)
$$

The discrete low-pass filter $h_{\text {low }}$ is associated with the scaling function. It was carefully chosen in such a way, that the filtering will reduce the width of the edges. It is given by a $5 \times 5$ matrix scaled by 256 :

$$
h_{\text {low }}=\left(\begin{array}{rrrrr}
1 & 4 & 6 & 4 & 1 \\
4 & 16 & 24 & 16 & 4 \\
6 & 24 & 36 & 24 & 6 \\
4 & 16 & 24 & 16 & 4 \\
1 & 4 & 6 & 4 & 1
\end{array}\right) .
$$

- Smoothed image is then used as a new input in the convolution, which is then repeated iteratively (100 times in our analysis in the Chapter6):

$$
c_{i}(x, y)=\sum_{l, m} h_{\mathrm{low}}(l, m) c_{i-1}\left(x+2^{i-1} l, y+2^{i-1} m\right)
$$

- The last smoothed image, i.e. in our case $c_{100}(x, y)$, is then subtracted from the original $c_{0}(x, y)-c_{100}(x, y)$ to give the desired edge enhanced image. It corresponds to the sum of all the high frequency components ("details" in Fig. 6.4).

Small-scale structures embedded in larger features are better seen after a multiple-scale based filtering, which is obtained when the scales are weighted using an appropriate reconstruction scheme. 


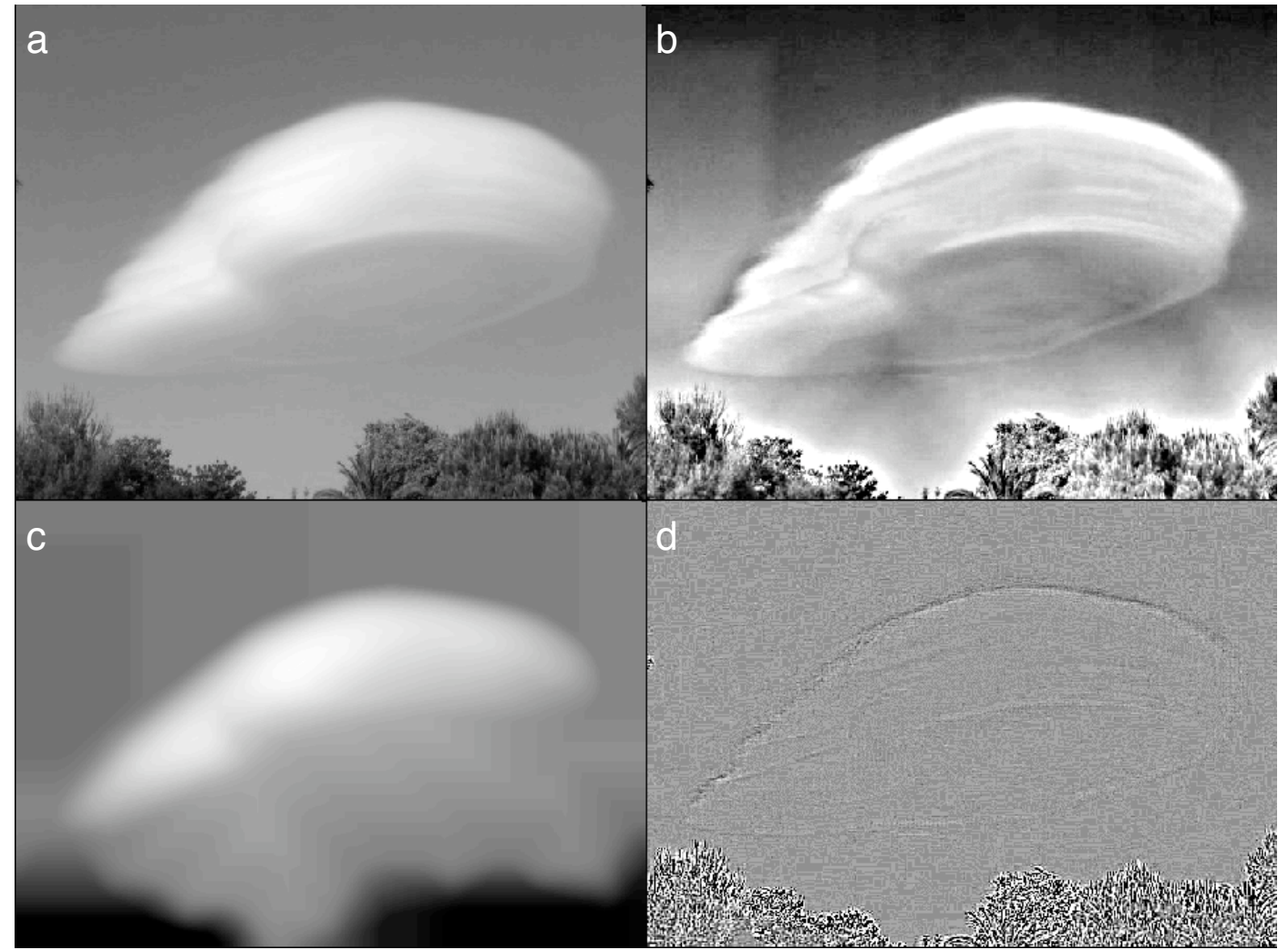

Figure 6.5: An original digital photo (a), an edge enhanced image using the single-scale filtering (b), a smoothed image (c) and the image with the highest frequency components (c).

The smoothed image in Fig. 6.5(c) is the last smoothed image in the iteration, so adding it to the enhanced image (b) will give the original photo (a). The detail image (d) is obtained after the first convolution of the original with the filter mask given by 6.5 . The enhanced edges are very well seen in the layers of the cloud, in the contrast of the trees and at the transitions between the dark trees, grey sky, and white cloud.

\subsection{April 2002 C Class Flare}

\subsubsection{Observations}

The flare occurred around 07:24 UT on 9 April 2002 in AR09886, the most northern of a complex of active regions near the western limb (Fig. 6.67). Faint coronal loops can be seen extending high in the corona. Some connect to AR09887 this side of the limb, 

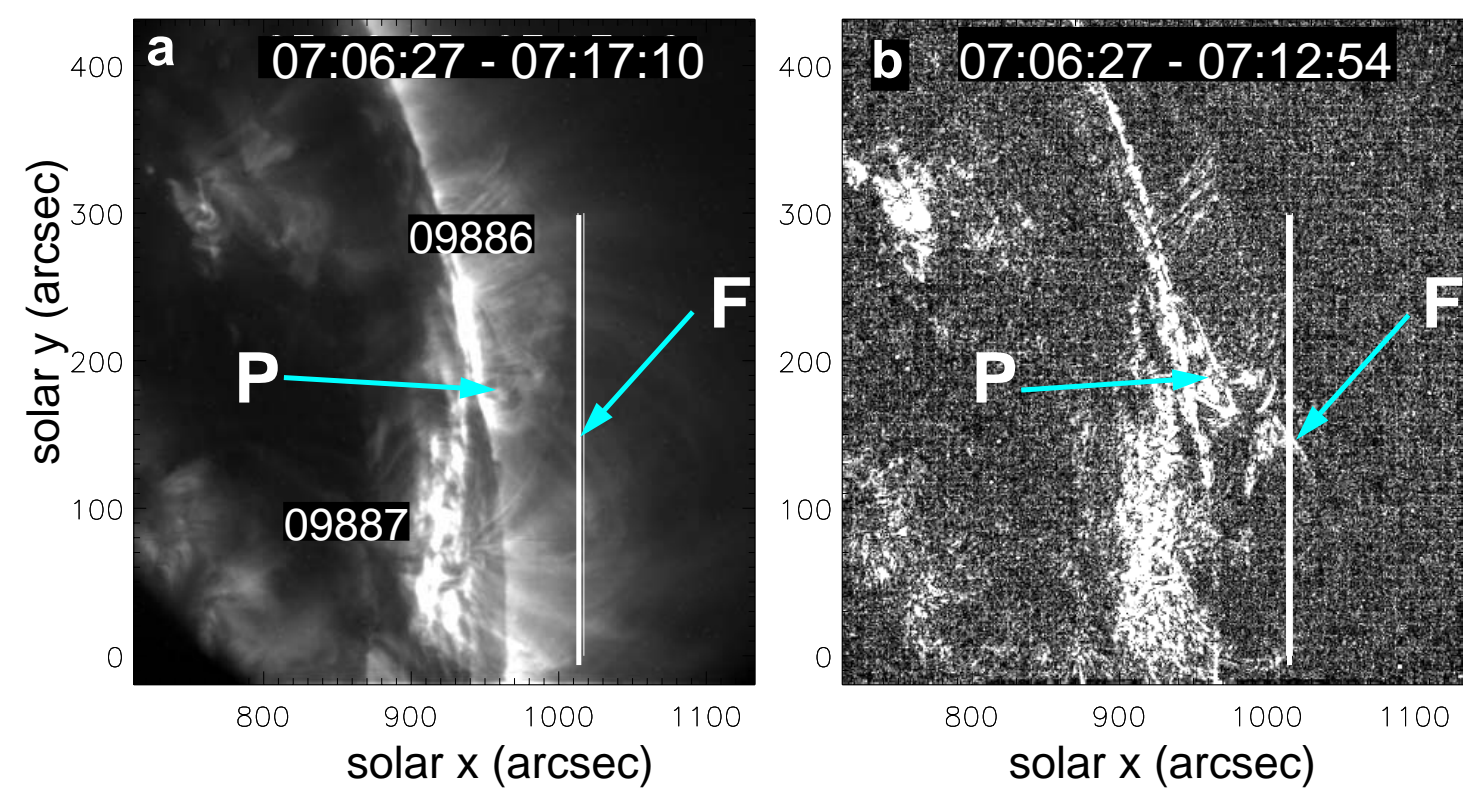

Figure 6.6: a) Average TRACE 195 image of AR09886 and surrounding region: (a) average intensity between 07:06 and 07:17 UT showing the prominence $(\mathrm{P})$ that later erupted; (b) power map for frequencies less than $5 \mathrm{mHz}$ (periods greater than $3.3 \mathrm{~min}$ ) between 07:06 and 07:12 UT. Flows (F) are detected in the corona crossing the SUMER slit which is indicated with white vertical line.

and others seem to be directed towards active regions, AR09885 and AR09891, already behind the limb. We show in Fig. 6.6 b, the power map of lowest frequency intensity variations in the pre-flare phase. The power maps were computed using Fourier analysis (see A.6) of intensity times series at each position in the image. This reveals activity in the prominence $(\mathrm{P})$ and flows $(\mathrm{F})$ in the corona.

An overview of the evolution of the loop system seen in TRACE, SUMER and Meudon is shown in the movie in Fig. 6.7, starting at 07:06 UT, the beginning of the TRACE observations. The movie shows simultaneous TRACE and SUMER intensity images, and $\mathrm{H} \alpha$ Dopplergrams in the top row. Below are the corresponding running difference intensity images. Each frame is scaled individually, thus hiding the time evolution of the intensity, which is represented in TRACE and SUMER time-distance images (Figs. 6.8 and 6.10, respectively). More details on each instrument as well as on the wavelength band in which it observes can be found below. 

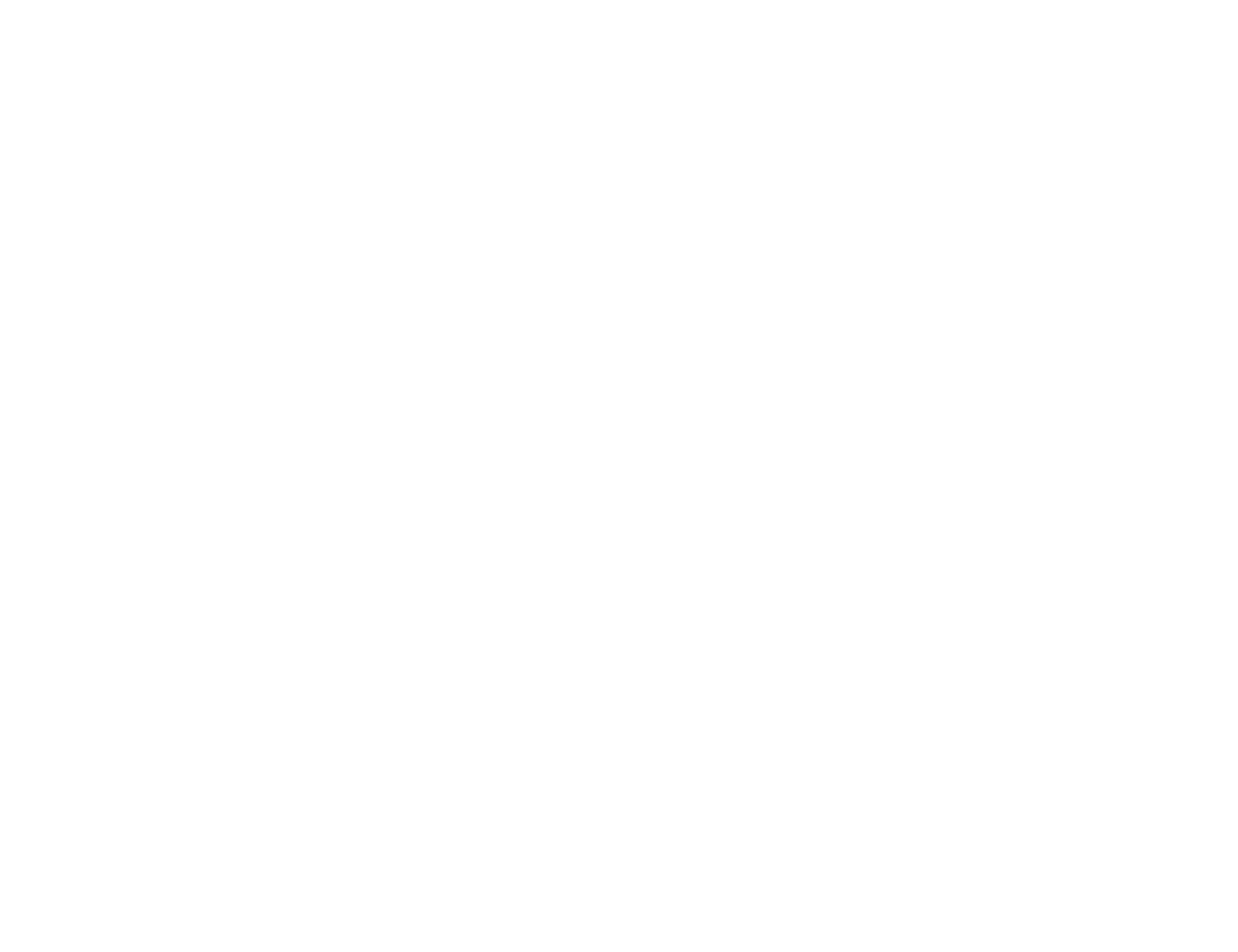

Figure 6.7: The flare evolution: (a) TRACE contrast enhanced intensity (b) SUMER spectral windows centered on Si III $1113 \AA$, Ca x 557 reverse color (c) Meudon Heliograph $\mathrm{H} \alpha$ Dopplergrams (d) TRACE running difference (e) SUMER running difference (f) $\mathrm{H} \alpha$ running difference. Two vertical lines indicate the position of the SUMER slit in the TRACE and $\mathrm{H} \alpha$ images. Clicking on $\triangleright$ will start the full movie.

\subsubsection{TRACE}

TRACE (Handy et al. 1999) observed in the $195 \AA$ band with a cadence of roughly $13 \mathrm{~s}$. The emission is due to Fe XII (1.6 MK) in the quiet Sun, and is dominated by emission

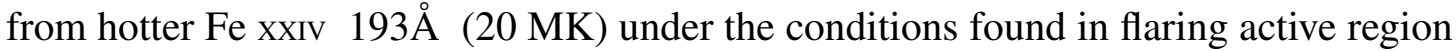
coronae (see Fig. 2.8). Coronal dimming at $195 \AA$ is due either to a decrease in coronal emission du to decreased emission or/and absorption by Hydrogen and Helium along the line-of-sight (see Section 4.3.1). In the filter band the transition region lines O v 192.8 and 192.9 (Young et al. 2007) may contribute to the observed prominence emission ( A.3.2). The TRACE data were first processed with the solarsoft routine trace_prep using the standard settings. Cosmic ray spikes have been removed. We then averaged 
all TRACE images, within each $50 \mathrm{~s}$ exposure period of SUMER, so that they overlap as much as possible in time. For the intensity movie (Fig. 6.7 a), the averaged images have been further contrast enhanced by subtracting a blurred image obtained by using repeated convolutions of a bi-dimensional mask (Stenborg \& Cobelli 2003) on the image (Section 6.2.2). The running difference movie uses the averaged data.

\subsubsection{Meudon Heliograph}

Images were obtained with the Meudon heliograph at a 1 min cadence in the core $(6563.3 \AA)$ and in the wings $( \pm 0.5 \AA)$ of the $\mathrm{H} \alpha$ line, the most prominent photospheric line (see the Section 2.1). The Doppler shift, Fig. 6.7k, is computed as the first weighted moment of the intensity (Eq. 2.13), using values in the core and wings of the line. The maximum measurable shift of $0.5 \mathrm{~A}$, when all the emission comes from one wing of the line, yields a maximum measurable velocity of about $23 \mathrm{~km} \mathrm{~s}^{-1}$ (Eq. 2.14). This is much lower than the velocities measured with the SUMER spectrometer. Any $\mathrm{H} \alpha$ emission coincident with the high velocity Si III plasma seen during the eruption was outside the filter window.

From around 07:20 UT, a prominence is seen to rise in the $\mathrm{H} \alpha$ intensity running difference images (Fig. 6.7f), showing faint Doppler shift signatures. The apparent planeof-sky velocity of the rising prominence was in the range $120-160 \mathrm{~km} \mathrm{~s}^{-1}$, significantly lower than the fast moving features seen in TRACE and SUMER data mentioned below. The erupting prominence started expanding towards Earth (blue shift), while its northern footpoint moved away (red shift), suggesting that the northern footpoint was already behind the limb and it preceded the southern one as the Sun rotates. It reached the height of the SUMER slit just after 07:24:33 UT.

\subsubsection{SUMER}

The SUMER spectrometer (Wilhelm et al. 1995) observed in sit-and-stare mode about 50 " off the west limb allowing the observation of loops formed within and among active regions NOAA AR09886, 09887, 09885 and 09891 with the 300" $\mathrm{x} 4$ " slit and a $50 \mathrm{~s}$ cadence simultaneously in the 3 lines: Si III $1113.24 \AA$ (0.06 MK), Ca x $557.76 \AA$ (second order, $0.7 \mathrm{MK})$ and $\mathrm{Fe}$ XIX 1118.1 $\AA$ (6 MK). The line formation temperatures are given in brackets. More details on the corresponding transitions and their blends are given in the Table A.3.1. The size of the spatial and spectral pixels was about 1 " and $44 \mathrm{~m} \AA$ respectively. The width of the spectral window around each line was $50 \mathrm{px}$, corresponding to about $2.2 \AA$, or in terms of the line-of-sight component of velocity, to approximately $590 \mathrm{~km} \mathrm{~s}^{-1}(\mathrm{Eq}$. 2.14). The data were flat-fielded, and geometrically corrected using standard procedures. Then the continuum intensity was subtracted. In the movie in Fig. 6.7p,e the three spectral windows, $\mathrm{Si}$ III , $\mathrm{Ca}$ x and Fe XIX , are plotted side by side with data gaps of $50 \mathrm{~km} \mathrm{~s}^{-1}$ and $140 \mathrm{~km} \mathrm{~s}^{-1}$ between the windows. 
The Fe XIx 1118.07 $\AA$ line blends with both transition region, $\mathrm{P} v 1117.98 \AA$ and Ne vi 558.62 $\AA$, and cooler $\mathrm{C}_{\mathrm{I}}$ lines at $1117.20,1117.58,1117.88,1118.18$, and $1118.49 \AA$ (Table A.3.1, Curdt et al. (2001)). We used the Si III to identify potential transition region blending. For example, in Fig. 6.7b, the strong emission around $y=230 "$ which coincides with strong $\mathrm{Si}$ III is Ne VI, but the emission below, between $150-200$ ", is Fe XIX . Here high Ne vi Doppler shift emission is seen beyond the $140 \mathrm{~km} \mathrm{~s}^{-1}$ data gap on the edge of the $\mathrm{Ca} \times$ window. The $\mathrm{C}_{\mathrm{I}}$ lines can be identified because another $\mathrm{C}_{\mathrm{I}}$ multiplet (1114.64, 1114.86 and 1115.21 $\AA$ ) appears at the same time in the $\mathrm{Ca} \mathrm{x}$ window. The analysis described here considers Fe XIX at times and positions when there is no strong Si III or $\mathrm{C}_{\mathrm{I}}$.

The coalignment of the SUMER observations with the TRACE images was not straightforward because of the many possible blends in both SUMER and TRACE. Key features used in the co-alignment were the position of the early pre-flare flows seen in TRACE and SUMER, the blue-shifted Ne VI , Si III and $\mathrm{H} \alpha$, seen as erupting prominence material in TRACE, and bright in TRACE, red shifted SUMER Si III and $\mathrm{H} \alpha$ along the northern leg of the erupting prominence at the end of the movie. SUMER was found to be centered at (1015", 150"), with an accuracy of 5" in both directions.

\subsubsection{Ejecta, Fronts and Oscillations}

The disruption of the corona is captured by the TRACE images. To obtain the expansion speeds of some of the observed fronts, we have constructed time-distance intensity images along horizontal synthetic slits (Fig. 6.8). Bright fronts with plane-of-sky speed greater than $350 \mathrm{~km} \mathrm{~s}^{-1}$ are seen throughout the region. According to the Equation 5.1 and the ideal gas law 3.23 , the speed of sound can be estimated to $380 \mathrm{~km} \mathrm{~s}^{-1}$. Taking the geometry corrections into account, the bright fronts propagate at a supersonic speeds. They seem to be associated with the eruption that started along $y=170$ " at 07:20 UT (Fig. 6.8p) in which both prominence and corona take-off simultaneously, similar to the mini-CME onsets reported by Innes et al. (2010). This suggests that the both eruptions seem to have the same trigger. Flows are seen before the eruption with TRACE in the corona crossing the SUMER slit (labeled F in Fig. 6.6p) and in the prominence (labeled $\mathrm{P}$ in Fig. 6.6p), which seems to be supported by overlying loops (Fig. 6.6a). This lead us to lean towards the interpretation based on the flux-rope model (see Section 4.4.2.2). Though to be sure, the pre-event magnetic topology (4.11) would have to be known. This is not the case, since it is a limb event and the geometry is not well understood. The northern leg was behind the limb at the time of the eruption thus hiding the footpoints of the loops. The prominence, also seen as dark fronts along $y=200 "$, reached the SUMER slit at 07:26 UT. 


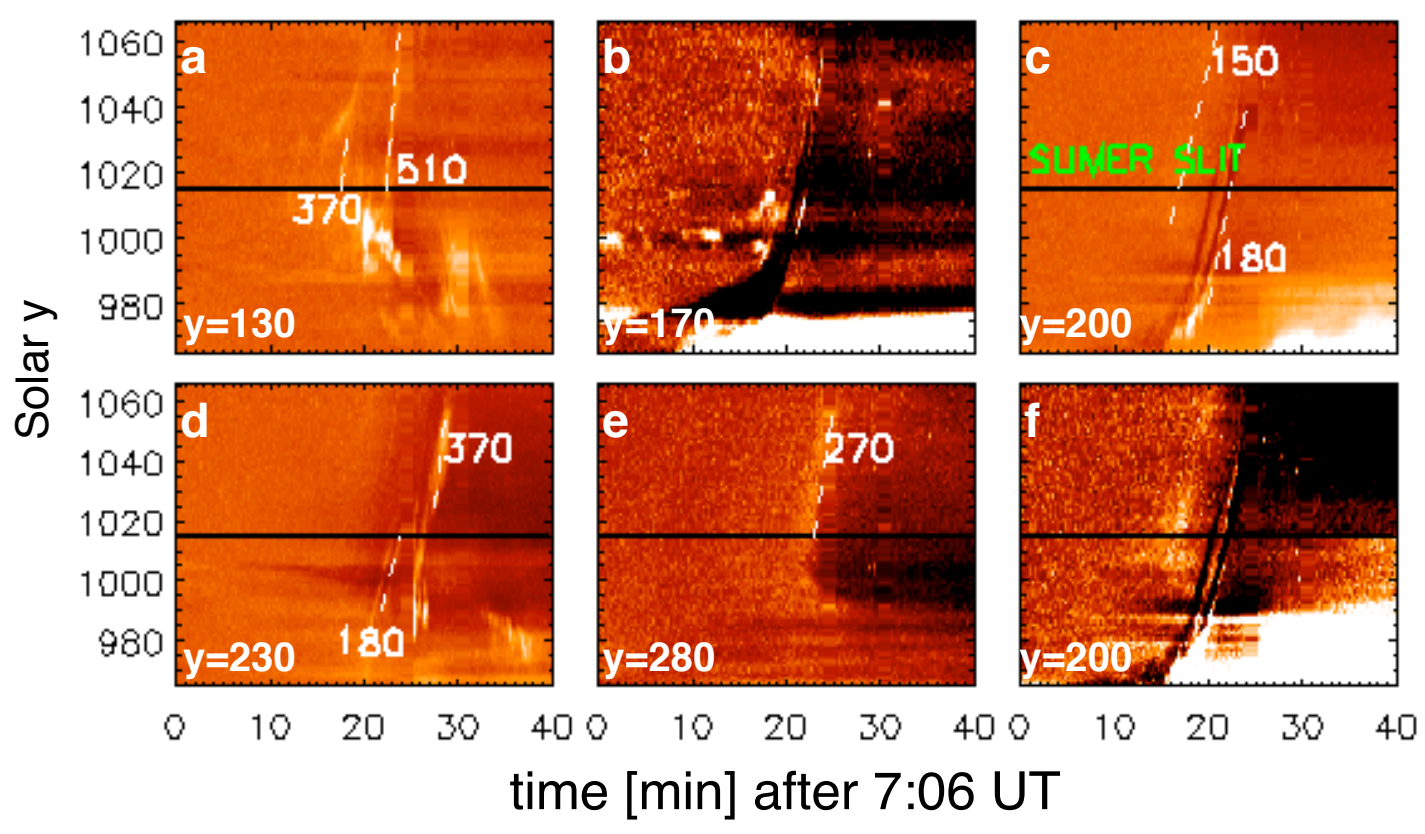

Figure 6.8: TRACE $195 \AA$ intensity synthetic slits constructed at different latitudes. The position of the SUMER slit, $x=1015$ ", is represented by the horizontal black line. Pre-event background intensities have been subtracted from each time series. Apparent plane-of-sky velocities in the direction of growing $x$ are given alongside dashed lines running along TRACE fronts.

North of $y=200 "$, one sees a bright front followed by coronal dimming. Because the front is barely visible in Fig. 6.8c, we present a re-scaled version of the figure below (6.8). The front is visible above the northern leg of the rising prominence. We suggest that this is the front of a blast wave similar to that discussed in Aschwanden et al.(2009). Its apparent horizontal velocity at $y=280 "$ is estimated to be around $270 \mathrm{~km} \mathrm{~s}^{-1}$.

Striking in the TRACE intensity frames of the movie in Fig. 6.7 but difficult to show with stills, is the large-scale back-reaction of the loop systems to the passing front. The magnetically dominated corona is strongly inhomogeneous. The loops and the cold prominence material present magnetic clouds with en enhanced density in comparison with the ambient plasma. Due to the highly structured and optically thin corona, where the emission along the line-of-sight adds up, the interaction in three dimensions is much more complicated. Since the magnetic loops form an elastic support for the waves, the oscillations are expected in the wake.

By making space-time images along different tracks in the corona, we were able to pick 

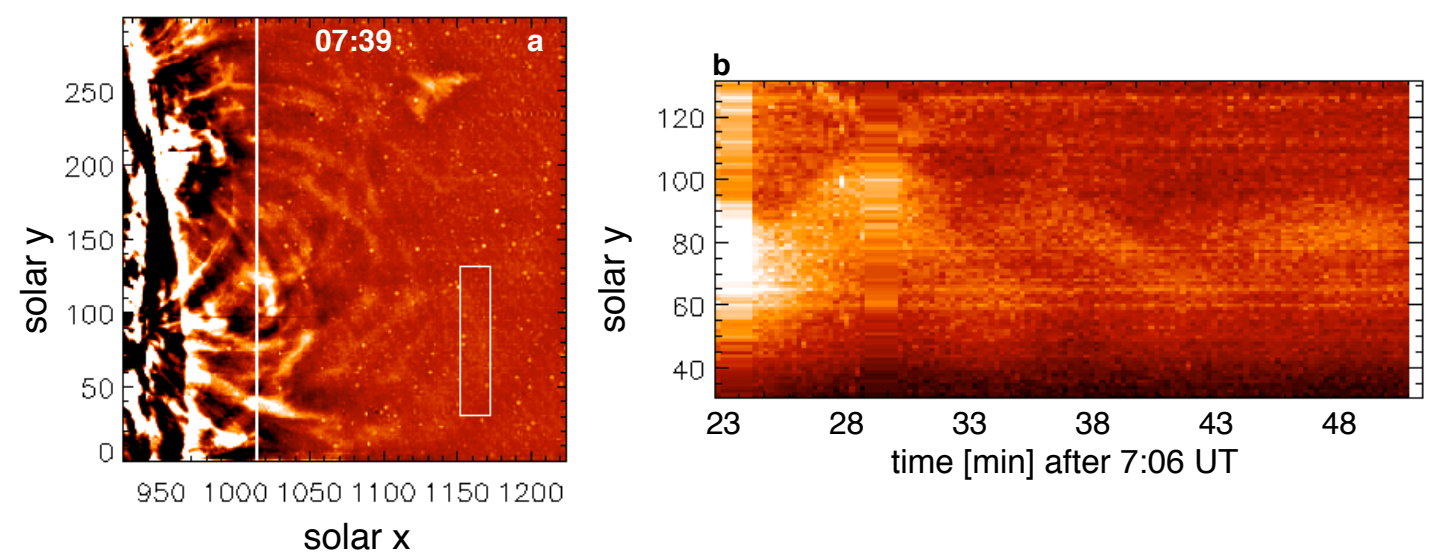

Figure 6.9: TRACE loop oscillation: a) TRACE contrast enhanced intensity image with the position of the SUMER slit (white vertical line) and the region of the oscillation (white rectangle) marked; b) time evolution of TRACE intensity averaged across the white rectangle in (a).

out the displacement oscillation shown in Fig. 6.9. It looks as though a broad, 20" wide structure, possibly a loop top, was oscillating up and down in latitude with a period of about $7 \mathrm{~min}$. This effect could either be caused by a loop top or the leg of a radially oscillating loop with an inclination angle of about $45^{\circ}$ to the limb. Since the oscillation in the movies is predominantly radial, the latter is more likely. As it is observed as a displacement oscillation, we suggest an interpretation in terms of the asymmetric kink mode (5.2.5). Estimating the length $L$ of the loops reaching the position of the oscillation to be around $500 \mathrm{Mm}$, the phase speed $\mathrm{v}_{\mathrm{ph}}$ can be calculated: $\mathrm{v}_{\mathrm{ph}}=2 \mathrm{~L} / \mathcal{P}=2380$ $\mathrm{km} \mathrm{s}^{-1}$. This value is of the order of the expected kink speed (5.4).

The SUMER Si III and Fe XIX time series in Fig. 6.10 show the spatial development of the transition region and hot flare plasma along a single vertical slit in the corona. The spectral details are best seen in the movie in Fig. 6.7. The Si III red shifts in the middle of the SUMER slit, correspond to velocities greater than $300 \mathrm{~km} \mathrm{~s}^{-1}$ from 7:06 UT. The early flows, directed southward and away from the observer, coincide with the coronal flows (F) seen in the TRACE power map (Fig. 6.6b). At 07:20 UT, the time of the two-tier eruption (Fig. 6.8 b), SUMER detected the coronal part of the disturbance. The transition region plasma moved rapidly to the north and south with an apparent planeof-sky velocity of $200 \mathrm{~km} \mathrm{~s}^{-1}$.

The large Si III red shifts seen later along the southern part of the SUMER slit, show up as flows in the TRACE running difference images of the movie in Fig. 6.7. The images suggest that the movement of $\mathrm{Si}$ III along the slit was not plasma motion but marks a front triggering Si III flows. In the north, we can associate the $450 \mathrm{~km} \mathrm{~s}^{-1} \mathrm{Si}$ III and 


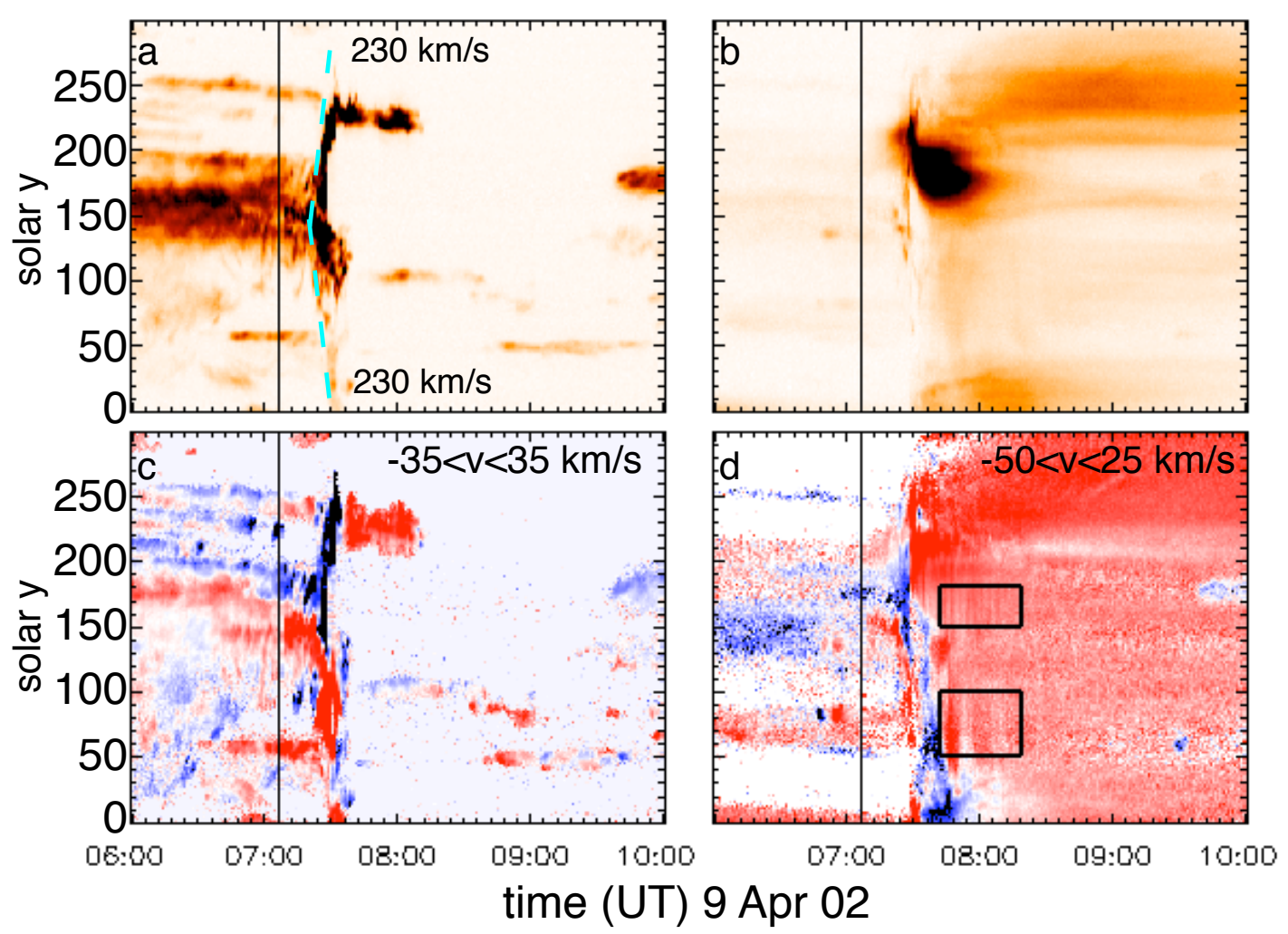

Figure 6.10: SUMER line intensities and Doppler shifts: (a) Si III intensity (b) Fe XIx intensity (c) Si III Doppler shift (d) Fe xIx Doppler shift. Intensities are reverse color. The start time of the movie is marked with a black vertical line. The rectangles in (d) outline the oscillation regions shown in Figs. 6.11, 6.12

Ne vi blue shifts seen in SUMER at 07:30 UT (Fig. 6.7) with erupting prominence plasma. Immediately after the erupting prominence, the Fe xIx intensity increased. It was particularly bright above the active region but significant emission also appeared to the south along the slit. Initially the Fe XIx line was predominantly blue shifted with a non-Gaussian wing extending to $150 \mathrm{~km} \mathrm{~s}^{-1}$.

Weak oscillations following the front can be seen in the Fe xIx space-time Doppler shift images (Fig. 6.10). Details of two of the regions, outlined by black rectangles in Fig. 6.10d, are shown in Fig. 6.11 a,b. The time span of the details is 33 minutes and is represented by the length of the horizontal side of the rectangle. In Fig. 6.11c,d, we show the wavelet power of the line center Doppler shift averaged over the 50" and 30" regions of the slit shown in the figure. It was calculated as the modulus of the wavelet transform with the Morlet wavelet (6.1) as the scaling function. The Doppler shift of the 
center of the line was calculated using Eq. 2.13 . The one to the south at $50 "<y<100 "$ has a single period of about $14 \mathrm{~min}$. The other at 150" $<y<220 "$ is more complex. It is composed of two periods, one around $4 \mathrm{~min}$ and the other also around $14 \mathrm{~min}$. No oscillation is seen in the region between.

The radius of the loops reaching the spectrometer is larger than $50 \mathrm{Mm}$, which corresponds to the height of the slit. If we assume a circular loop, then the speed $\mathrm{v}_{\mathrm{ph}, 4}=2 \mathrm{~L} / \mathcal{P}>1300 \mathrm{~km} \mathrm{~s}^{-1}$ for the 4 minute period, and $\mathrm{v}_{\mathrm{ph}, 14}>373 \mathrm{~km} \mathrm{~s}^{-1}$ for the 14 minute period. Therefore, we suggest, that the blast wave gives rise to the standing kink mode (Section 5.2.5), when it hits the loops edge-on, and a slow mode (Section 5.2.6), when the trigger is situated near one of the footpoints.

In Fig. 6.12, we plot the time evolution of the spectra averaged over 10" and centered at the strongest points of the oscillations. Vertical black lines denote the start of the time span of the oscillations, 7:42 - 8:22. The transition region Si III emission disappeared before the Fe XIX oscillations, so we conclude that the oscillation is definitely due to the shifts in Fe XIX line and not to contamination by its colder blends (see Table A.3.1).

The spectral evolution shown in Fig. 6.12 is typical for the positions $50 "<y<100 "$ in which we see several such Si III jets followed by an Fe XIX oscillation starting with a blue shift. It seems to support the impression given by the images that the $\mathrm{Si}$ III flows were triggered by a front moving through the corona because a large-scale front would also heat plasma in its wake. It is not clear whether the Fe xix blue shift was caused by a back reaction to the red shifted Si III jets or it was different plasma accelerated behind a part of the front moving towards the Earth. 

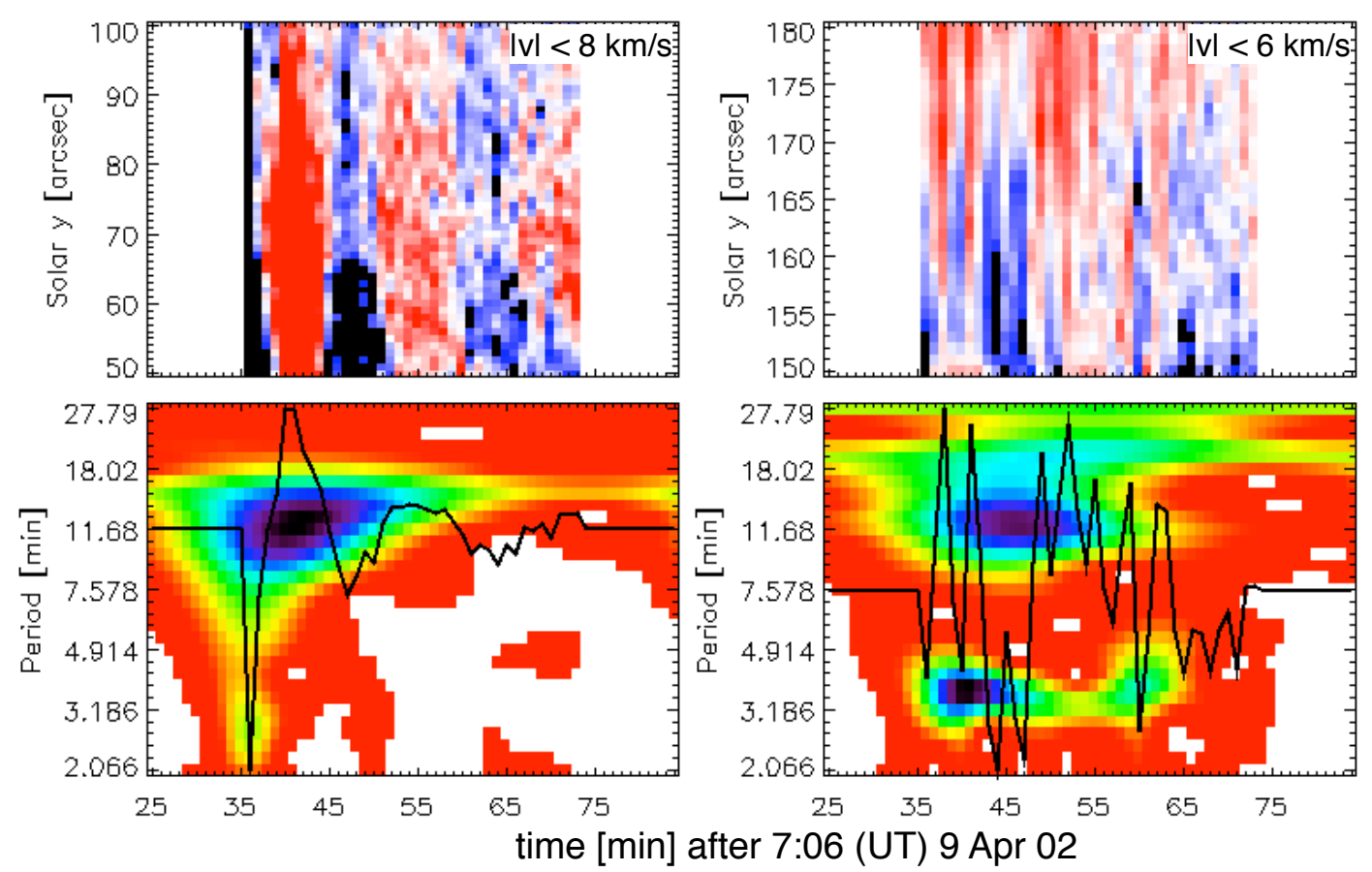

Figure 6.11: Detail of the Doppler shift oscillation in Fe XIX for (a) The lower rectangle in Fig. 6.10 d, and (b) the upper rectangle in Fig. 6.10 d. The frames underneath show the wavelet power of the Doppler shifts in the frame above with the average Doppler shift overplotted.

\subsection{April 2002 M Class Flare}

\subsubsection{Introduction}

The oscillations discussed here are seen in flare loops and the ambient corona in both TRACE $195 \AA$ filter images and SUMER Fe XIX spectra during and after the passage of an erupting loop. The flare of GOES X-ray class M2.5, accompanied by a CME, occurred very close to the northwest limb. It was observed by several instruments, including SUMER, CDS, TRACE, RHESSI, LASCO and the Nancay radioheliograph. Sui et al. (2004), Goff et al. (2005), and Wang et al. (2007) suggest an interpretation based on the standard CSHKP (Carmichael 1964, Sturrock 1968, Hirayama 1974, Kopp \& Pneuman 1976) model of CME eruption because they saw an EUV loop slowly rising into the corona very closely followed by a small region of 5-25 keV X-ray emission. A short distance behind, Wang et al. (2007) saw large blue and then red Doppler shifts in Fe XIX. They interpreted them as direct observations of fast magnetic reconnection 

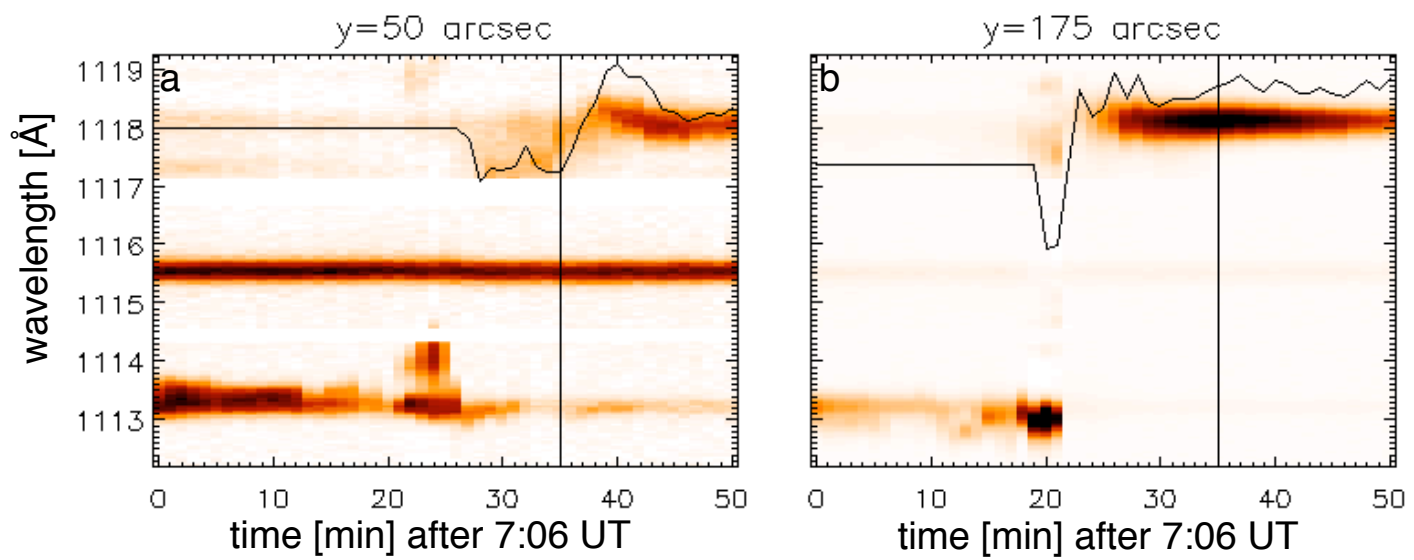

Figure 6.12: Spectral evolution at positions of the Doppler shift oscillations. The spectra have been averaged over 10" and displayed with the Fe XIX window at the top, $\mathrm{Ca} \mathrm{x}$ in the middle and Si III at the bottom. A black vertical line has been drawn at the start of the measured Fe XIX oscillations.

outflows along the current sheet.

We present a re-analysis of the SUMER spectroscopic and TRACE imaging observations. Through a careful study of the spectroscopic images, we show high blue and red Doppler shifts and broadening in earlier frames and over a significantly larger area and lasting longer than has been reported by Wang et al. (2007). We show that the slowly rising EUV loop is preceded by large Doppler shifts and is followed by an oscillation. The high velocity shifts as well as the oscillatory behaviour are significant far beyond the edge of the rising loop. We propose that the observations show heating and acceleration of plasma in the wake of a flare blast wave.

\subsubsection{Observations}

The flare started with a small soft and hard X-ray burst at 12:53 UT, followed by a stronger pre-impulsive burst at 13:04 UT. The impulsive phase started at 13:06 UT with a series of 5 or 6 strong hard X-ray bursts followed by an increase in soft X-ray emission. A coronal soft X-ray source, co-spatial with an EUV loop, was seen rising above the main post-flare loops from 13:03 to 13:07 UT with a speed $\approx 60 \mathrm{~km} \mathrm{~s}^{-1}$ (Goff et al. 2005). An increase in Fe XIX emission at a distance of around 50" from the limb started at around 12:56 UT (Wang et al.2007). A brief maximum in Fe XIX emission was seen when the EUV loop crossed the slit's field-of-view. Following the loop's passage the emission in the Fe XIX line and at $195 \AA$ decreased. This is followed by a progressive increase of intensity as the hot, dense cusp-shaped loops form and grow. 
The SUMER spectrometer (Wilhelm et al. 1995) observed in sit-and-stare mode about 50" off the west limb above the active region NOAA AR 9901 with the 300" $\mathrm{x} 4$ " slit and a 50 s cadence simultaneously in 3 lines: Si III $1113.24 \AA$ (0.06 MK), Ca x $557 \AA$ (second order, $0.7 \mathrm{MK}$ ) and Fe XIx 1118.1 $\AA$ (6 MK) (Fig. 2.9). The size of the spatial and spectral pixels is about 1 " and $44 \mathrm{~m} \AA$ respectively. The width of the spectral window around each line is $50 \mathrm{px}$, corresponding to about $2.2 \AA$, or in terms of the line-of-sight component of velocity, to approximately $590 \mathrm{~km} \mathrm{~s}^{-1}$. Thus, the Fe XIX window, being centered about 3 px towards the red wing, measures velocities in the interval from $-260 \mathrm{~km} \mathrm{~s}^{-1}$ to $+330 \mathrm{~km} \mathrm{~s}^{-1}$, where the negative sign stands for blue component. To detect blue Doppler shifts in Fe XIX with velocity greater than $400 \mathrm{~km}$ $\mathrm{s}^{-1}$, two neighbouring spectral windows, $\mathrm{Ca} \mathrm{x}$ and $\mathrm{Fe}$ xIX, were plotted side by side (Fig. 6.14 and 6.15) with a data gap between -260 and $-400 \mathrm{~km} \mathrm{~s}^{-1}$. No response to the event was seen in either intensity or Doppler shift of the Si III and $\mathrm{Ca} x$ lines.

TRACE observed in the $195 \AA$ band with a cadence of roughly $20 \mathrm{~s}$. The emission is due to Fe XII (1 MK) in quiet Sun, and is dominated by emission from the hotter Fe XXIV $192 \AA$ (20 MK) under the conditions found in flaring active region coronae. After the standard image corrections using SolarSoft routines a wavelet-based denoising technique using a 2-dimensional à trous algorithm (Stenborg \& Cobelli 2003, Stenborg et al. 2008) has been applied in order to bring up the loops in better contrast (Fig. 6.14a,c,e, g and 6.15a).

The coalignment of the position of the SUMER slit with the TRACE images has been obtained by comparing the common features seen in the intensity time series of the SUMER lines Fe XIX 1118.1 $\AA$ and Ca x $557 \AA$ and in the TRACE $195 \AA$ time series constructed at the position of the SUMER slit. Fig. 6.13 shows the final coalignment of the Fe XIX and the $195 \AA$ intensities with the pre-flare emission subtracted. The crescent shape corresponds to the mirror image of the rising loop observed by TRACE as it crosses the slit. As can be seen the maxima of the TRACE and SUMER intensities superimpose. SUMER is found to be centered at (934.5", 405.5"), with an accuracy of $5 "$ in both directions. 


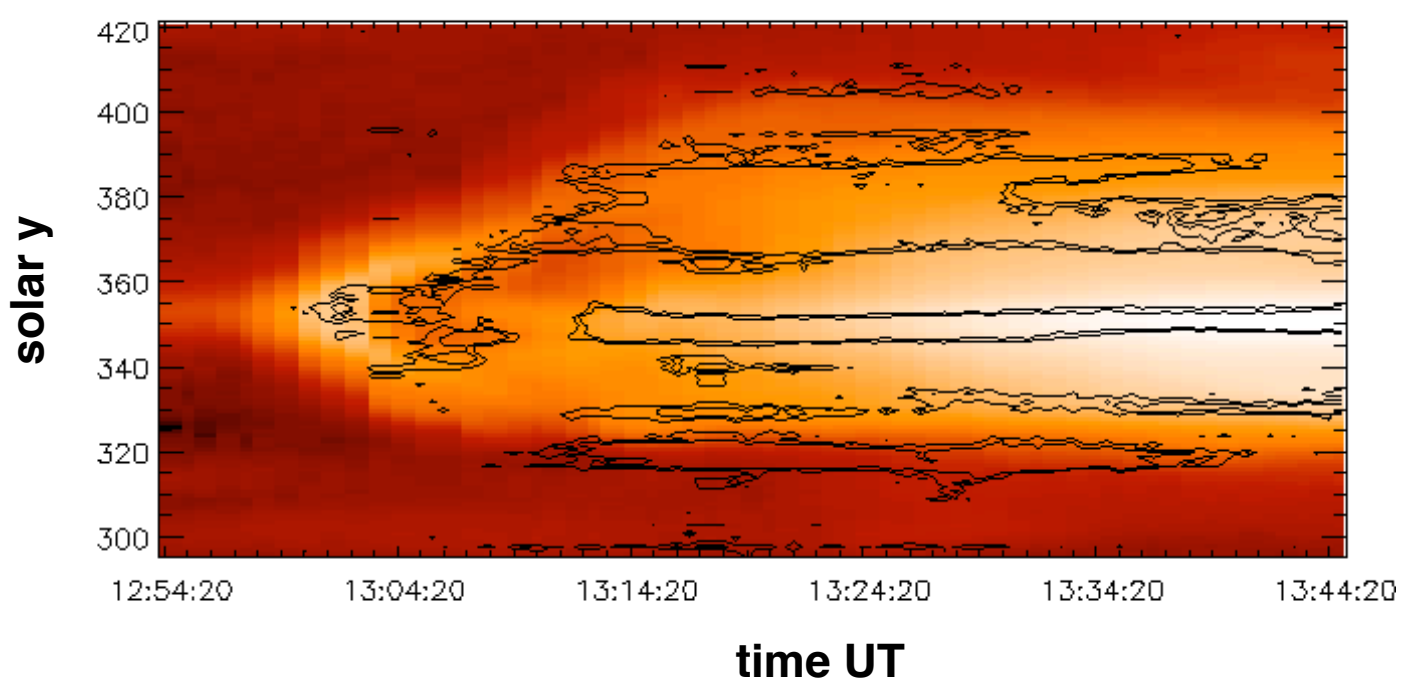

Figure 6.13: Fe XIX 1118.1 $\AA$ intensity time series on a logarithmic scale with TRACE $195 \AA$ intensity contours.

An overview of the loop system is shown in the TRACE frames in Fig. 6.14a,c,e,g and 6.15 . The position of the SUMER slit is indicated by the white vertical line. Alongside each TRACE $195 \AA$ intensity image, constructed by averaging over 3 successive frames, the Fe XIX 1118.1 $\AA$ spectrum closest in time is displayed (Fig. 6.14 b,d,f,h) and $6.15 \mathrm{~b}, \mathrm{c}$. The spectra in Fig. $6.15 \mathrm{~b}$ are represented on a linear scale, using the reversed rainbow color table, where the emission grows from white, through red and green to blue (b). The spectra represented in (Fig. 6.14 $\mathrm{p}, \mathrm{d}, \mathrm{f}, \mathrm{h}$ and 6.15 c) are running difference images obtained subtracting the earlier spectra from the ones specified in time. They allow us to view the changes better, as well as to bring out the faint high velocity shifts which usually occur before and on the edge of the bright Fe XIX line core. Each frame of the time series is scaled individually, thus hiding the time evolution of the intensity. The latter is represented in Fig. 6.13,6.17, 6.18 and 6.16.

A blue-shifted jet with a velocity greater than $200 \mathrm{~km} \mathrm{~s}^{-1}$ appears near $y=350$ " at 12:55:22 UT (green arrow in Fig. 6.14d). Its position corresponds to the top of the rising loop passing through the SUMER slit at 13:01:30 UT and its velocity increases up to $650 \mathrm{~km} \mathrm{~s}^{-1}$ between 13:05:37 and 13:11:40 UT (upper green arrow in Fig. 6.15b). Some 10" above it, red shifts with peak velocities up to about $250 \mathrm{~km} \mathrm{~s}^{-1}$ are seen from 13:01:30 UT, lasting until 13:19:05 UT (white arrow in Fig. 6.15c). Only the central high blue shift between 13:04 and 13:14 UT and central red shift after 13:16 UT were included in the Wang et al. (2007) analysis. In the movie, there is also a number of other high velocity blue-shift structures. The strongest starts around 12:59 UT below the 
TRACE $195 \AA$
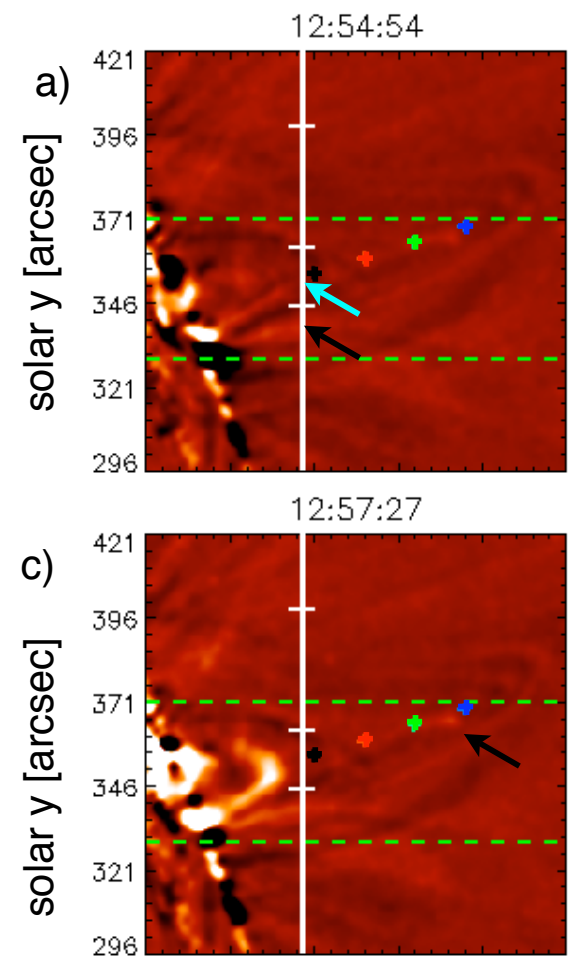

$13: 02: 02$
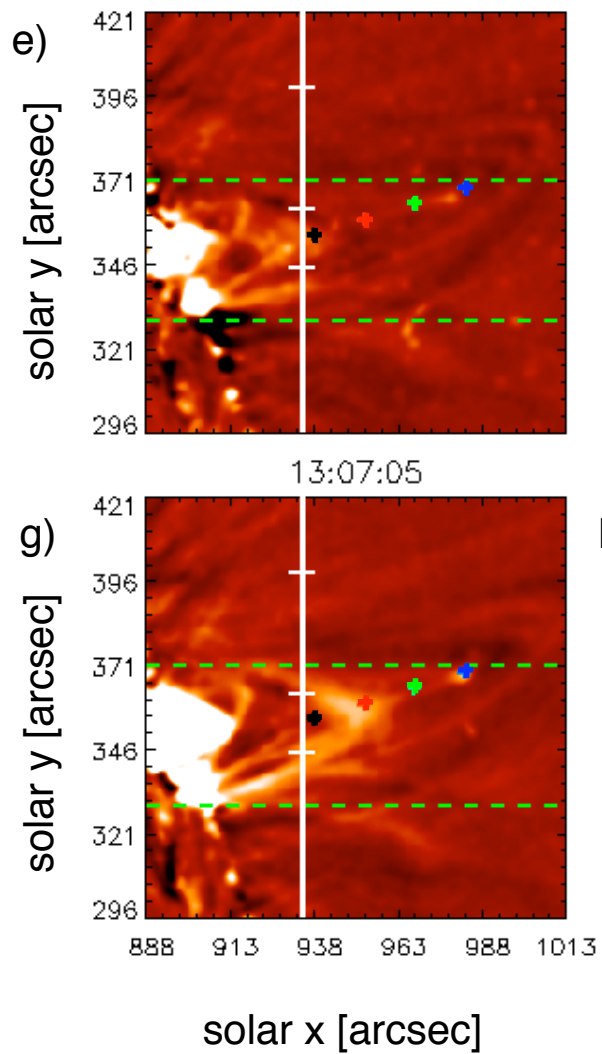

SUMER Fe XIX 1118.1

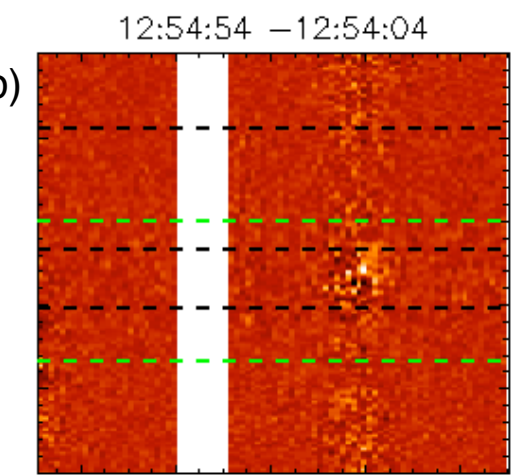

$12: 57: 22-12: 56: 33$

d)

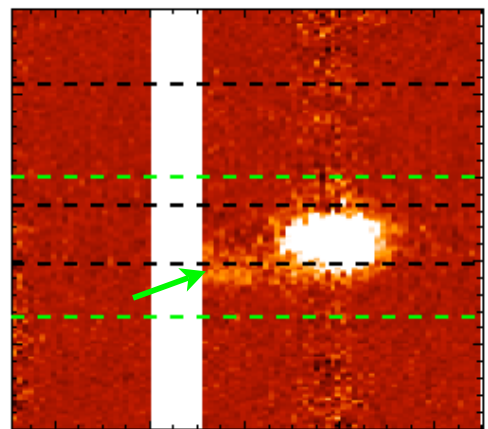

$13: 02: 19-13: 01: 30$

f)

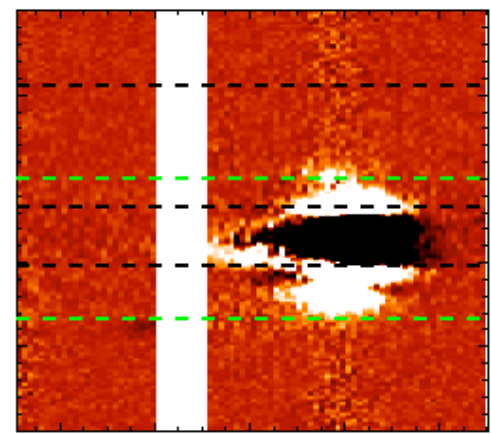

$13: 07: 32-13: 05: 37$

h)

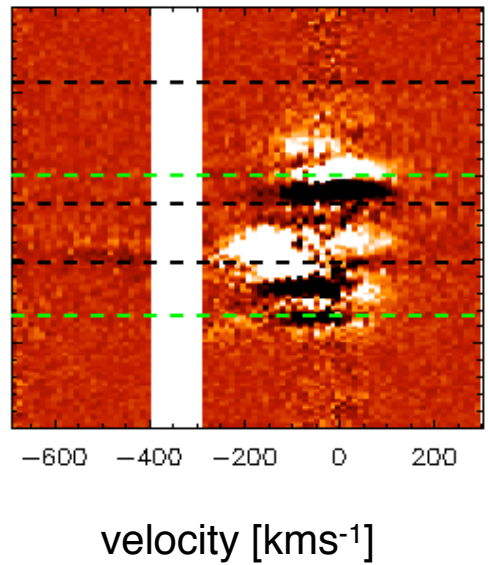

Figure 6.14: Left panels (a,c,e,g): same as left panel (a) in Fig.6.15.

Right panels (b,d,f,h): Same as right panel (c) in Fig. 6.15.

The green arrow (d) points at the position of the blue-shifted jet with a velocity greater than $200 \mathrm{~km} \mathrm{~s}^{-1}$ which appeared near $y=350$ " at 12:55:22 UT and then moved southward. The cyan and black arrows (a) point at the loops (corresponding arrows in Fig. 6.16b). 


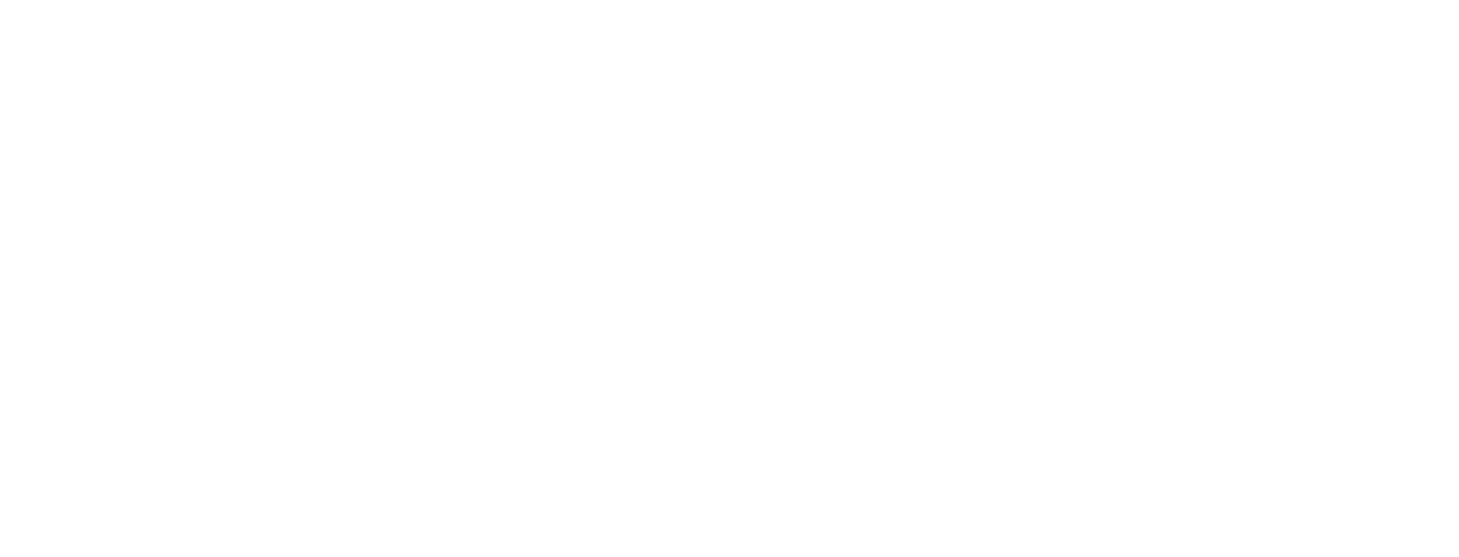

Figure 6.15: Left panel (a): TRACE $195 \AA$ intensity, enhanced and cleaned using a wavelet-based method. The white vertical line indicates the position of the SUMER slit. Black, red, green, and blue crosses represent the positions at which the intensity time series are plotted in Fig. 6.18. The three horizontal white lines on the slit designate the positions at which the time evolution of the Fe XIX Doppler shift and intensity, and TRACE $195 \AA$ intensity are compared in Fig. 6.17. The two horizontal green lines denote the width of the area represented in Fig. 6.16.

Middle panel (b): spectra of the emission line Fe XIX $1118.1 \AA$ along the spectrometer slit (vertical axis) as a function of wavelength relative to the center of the line, converted into line-of-sight velocity (horizontal axis). Each frame is scaled individually. Blue represents the highest emission at a given time.

The three dashed black lines are drawn at the slit positions marked by white horizontal lines.

Right panel (c): Running difference images of the spectra of the emission line Fe XIX 1118.1 $\AA$ along the spectrometer slit. White color represents the biggest emission increase, and black shows the highest emission decrease recorded since the previous frame (i.e. around 50 seconds earlier). 
rising loop (the bottom green arrow in Fig. 6.15b), moving southwards and increasing in its velocity. It reaches its maximum velocity, close to $500 \mathrm{~km} \mathrm{~s}^{-1}$, between 13:02:19 and 13:05:37 UT. At 13:10:50 UT, the blue shifts with velocities $250-600 \mathrm{~km} \mathrm{~s}^{-1}$ are seen over the largest portion of the slit outside the loop (from around 326" to 390").

SUMER observations show a Doppler shift oscillation of the center-of-gravity of the Fe XIX emission line. The extent of the oscillation in space and time shows up best in the surface plot of Doppler shift (Fig. 6.16). Although the large-scale oscillation is continuous there are variations in power, amplitude and frequency along it. In the TRACE intensity (Fig. 6.16p) the oscillation is also visible but because the intensity variation represents the superposition of real changes in temperature, density and ionization and new material moving into the field-of-view, the oscillatory behaviour may be more or less hidden at a given latitude. The black arrow in Fig. 6.16 b points to the stationary loop seen by TRACE, situated at the position of $y=337 "$. The loop is also indicated with a black arrow in Fig. 6.14 a. At the position of the cyan arrow $(y=348 ")$ in Fig. 6.16b, there is a faint loop (cyan arrow in Fig. 6.14 ) crossed by the SUMER slit which starts brightening up at 13:12 UT, reaching its maximum at around 13:18 UT.

The relationship between the Doppler shift and intensity oscillation is better illustrated in Fig. 6.17, which shows the evolution of the Fe xIX and $195 \AA$ intensity, as well as the Fe XIX Doppler shift at the positions of the white horizontal lines on the slit and black dashed lines on the spectral images in Fig. 6.14 a,c,e,g and 6.15 $\mathrm{k}$. The oscillation period varies between 11 and 14 minutes and the initial large red-shifted pulse indicates an impulsive trigger. At the positions either side of the region crossed by the apex of the loop, $y=345 "$ and 362" (Fig. 6.17p and c), the Doppler shift peak (blue curve) precedes the Fe XIX intensity peak (black curve) corresponding to the rising loop imaged by TRACE. Even 30" north of the loop ( $y=398 "$, Fig. 6.17 $)$, signatures of the oscillation in Doppler shift, starting at the time of the Fe XIX intensity increase, are seen.

To illustrate emission changes along the path of the apex of the rising loop, the $195 \AA$ intensity is plotted in Fig. 6.18 at the positions indicated by the black, red, green and blue crosses in Fig. 6.14 a,c,e,g and 6.15a using the corresponding colours. At the positions (938", 354") (black) and (953", 359") (red), the first peak in the $195 \AA$ intensity corresponds to the rising loop and its estimated propagation speed along this path is $45 \mathrm{~km} \mathrm{~s}^{-1}$. The second maximum does not propagate along the path denoted by the four crosses but rather arises simultaneously at all four positions. The increase in intensity on the loop's axis below the apex is followed by an increase on each side, propagating in the direction perpendicular to the path denoted by the crosses. This is the reason why the second peak at the position of the black cross (Fig. 6.18) is observed before the second peak at the positions below and above it (red curves in Fig. 6.17b and c). We suggest that this increase is due to the rapid changes in temperature and ionization of the plasma enhanced by the fast twisting and transverse motion of the loops, caused by the blast wave passing through. Around the position of the green cross, the first intensity peak is 


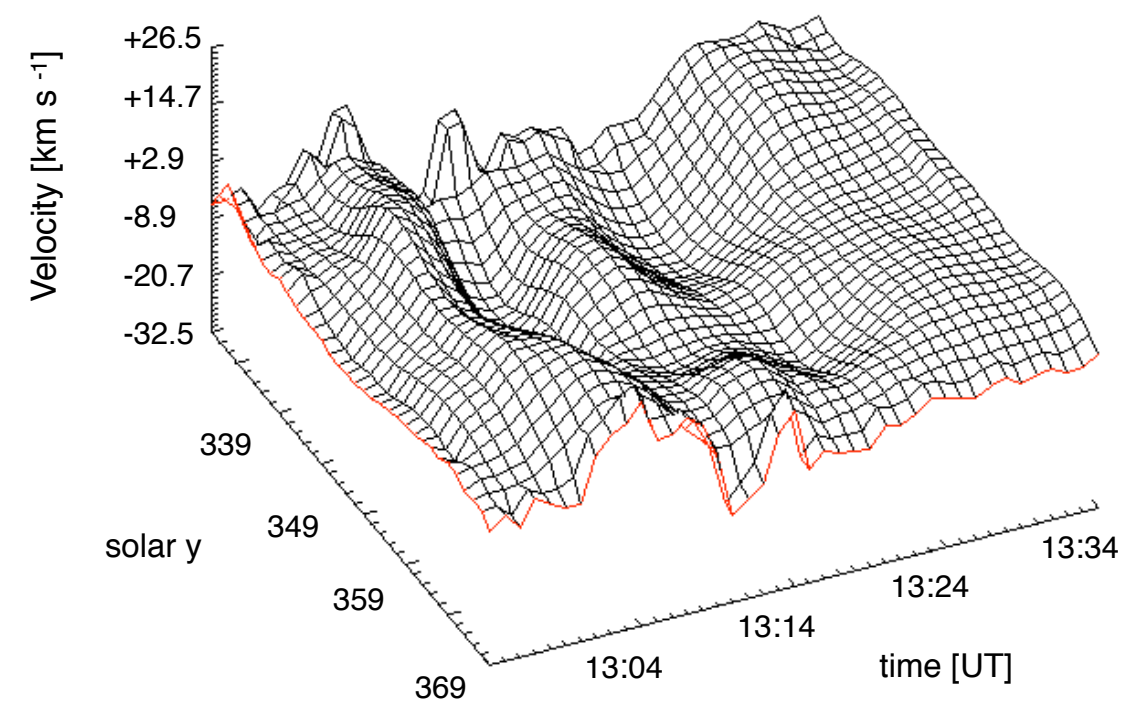

(a) Doppler shift oscillation of Fe XIX $1118.1 \AA$ line center.

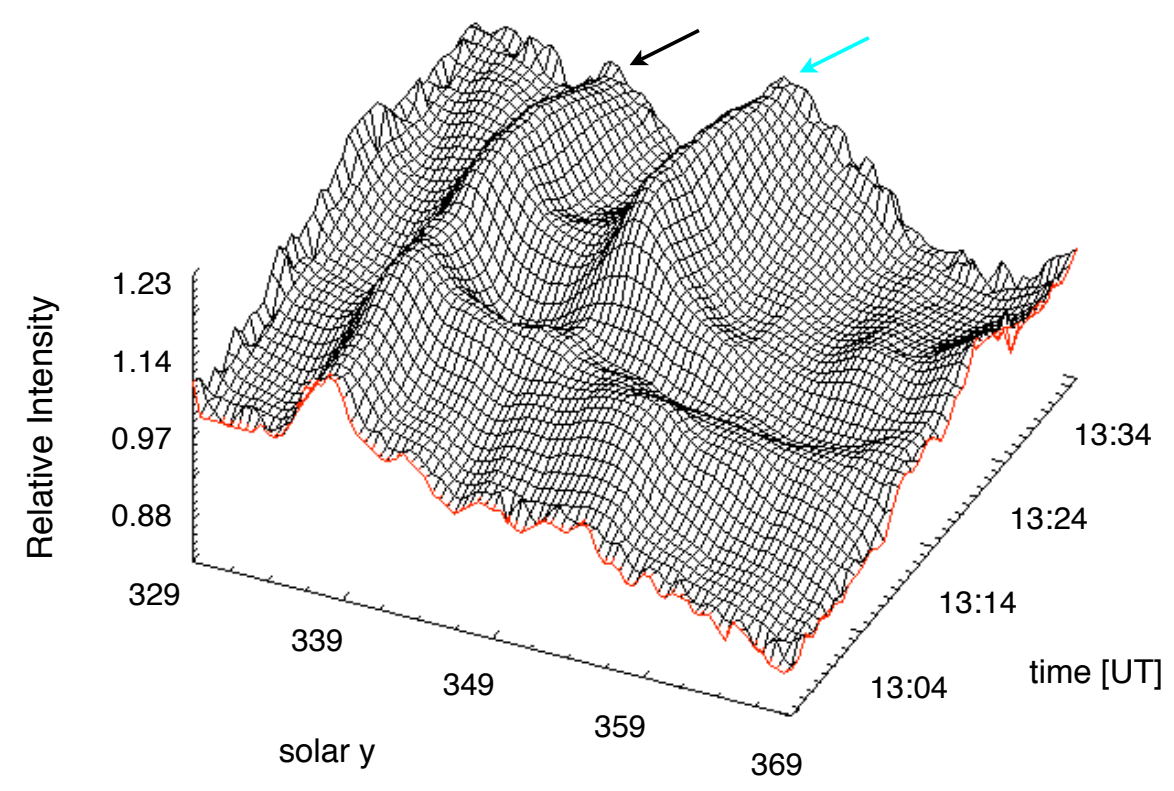

(b) $195 \AA$ intensity oscillation.

Figure 6.16: Doppler shift oscillation of the Fe XIX 1118.1 $\AA$ and the $195 \AA$ intensity at the position of the slit. The width of the comprised area along the slit is represented by 2 green horizontal lines in Fig. 6.14 and 6.15 
a)

solar $\mathrm{y}=398$

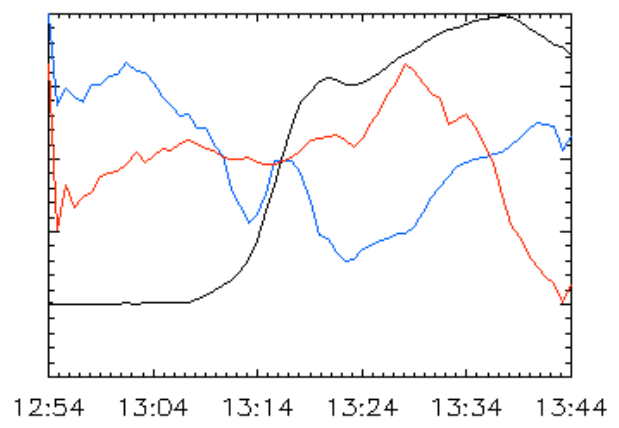

b)

solar $\mathrm{y}=362 "$

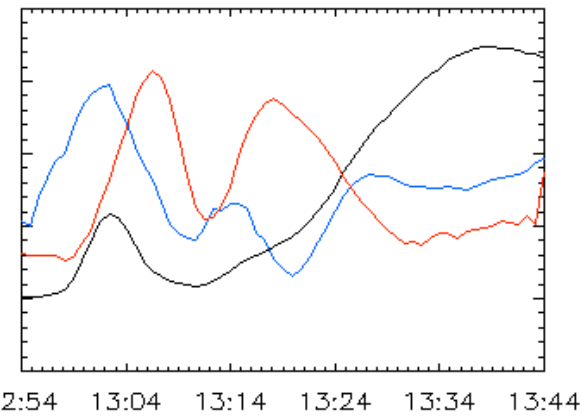

c)

solar $\mathrm{y}=345^{\prime \prime}$

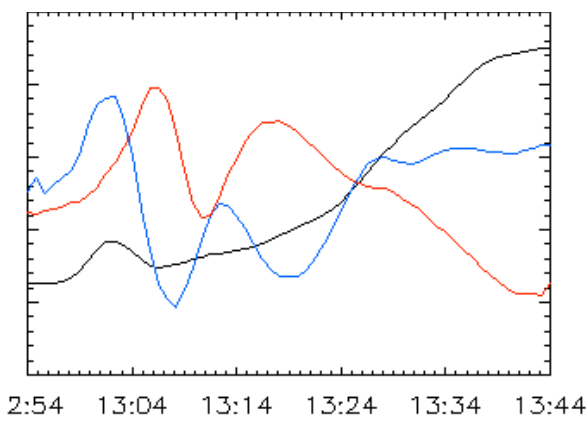

Figure 6.17: Relative modulation of intensity (black) and Doppler shift of the 1118.1 $\AA$ line center (blue), and TRACE $195 \AA$ intensity (red), at the positions on the slit indicated by white horizontal and black dashed lines in Fig. 6.14 and 6.15. The $195 \AA$ intensity is obtained by averaging over the width of the slit. All three curves are normalized individually for the purpose of a better comparison. 


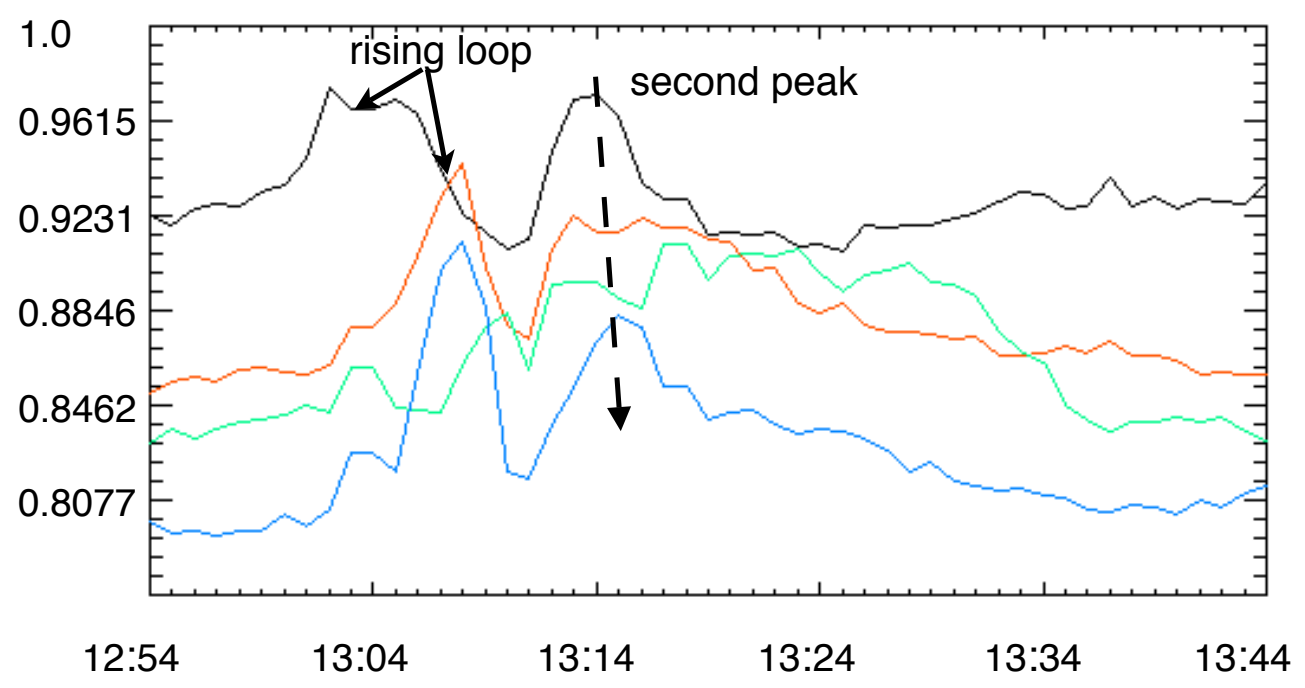

Figure 6.18: $195 \AA$ intensity oscillation. The four curves correspond to the relative intensity modulations at the positions indicated by crosses in Fig. 6.14a,c,e,g and 6.15a.

barely visible and, moving further outwards, it disappears completely, suggesting that the rising flux rope erupts at around this height. At the position indicated by the blue cross, two peaks are observed again. The first one occurs almost simultaneously with the first peak corresponding to the red cross, i.e. before the rising loop would have time to reach it if it didn't erupt. It is due to heating and an upward motion of a bright feature indicated by a black arrow in Fig. 6.14 . It is most probably the apex of a twisted loop whose legs are nearly aligned along the line of sight. It appears as a stationary source from 12:54 - 13:03 UT, then moves slowly upward in the same direction as the rising loop to the position (988", 367"). At around 13:10 UT it seems to disappear, probably due to heating caused by the passing blast wave, and reappears again at around 13:12 UT, at the position of (975", 371"). its apparent plane-of-sky velocity is thus around $80 \mathrm{~km} \mathrm{~s}^{-1}$ (13" in $\left.2 \mathrm{~min}\right)$. We suggest that its motion is due to a blast wave hitting it edge-on, much like the transversal oscillation of the loops.

\subsection{Discussion and Conclusions}

We present EUV spectroscopic and imaging observations of two flares, both accompanied by a large-scale wave, an oscillation in its wake, and a prominence eruption that was seen later as a CME in LASCO images. Here we summarize the arguments for the interpretation of the data in terms of heating and acceleration in the wake of a blast 
wave flowing around magnetic obstacles - coronal loops - formed within and among the active regions covering a large region on the Sun.

Multi-wavelength analysis of the loop dynamics before, during and after the April 9th $2002 \mathrm{M}$ class flare accompanied with a filament eruption and a CME have shown that:

- A bright front with a plane-of-sky velocity $250 \mathrm{~km} \mathrm{~s}^{-1}$ was seen ahead of the erupting filament in TRACE $195 \AA$ images. As the front passed through the SUMER slit, the Si III line brightened and brief bursts of red shifted emission were seen from positions moving south and north along the slit. The $\mathrm{Si}$ III intensity then decreased rapidly. At the same time, the intensity of the flare line Fe xIx increased and a Doppler shift oscillation lasting at least 1 hour was launched along broad sections of the slit, with a varying amplitude and period.

- The disappearance of Si III was either due to heating or ejecta moving out of the slit field-of-view. TRACE images provide clues to what happened. Initially, TRACE shows many bright ejecta moving south intermingled with a background dimming. Afterwards we saw increased Fe xIX emission, implying heating, and oscillating loops across the region, implying plasma acceleration on a large scale. We therefore suggest that the Si III flows are triggered by a front moving through the corona.

- At least three different oscillations detected with SUMER and TRACE, seen at different positions. SUMER shows large regions with 14 min oscillations at 50" $<y<100 "$ and 150" $<y<180 "$, which we interpret as the slow magnetoacoustic mode. We suggest, that the shorter period (4 min) oscillation superposed on the 14 min one in the northern section is a kink mode. A TRACE kink oscillation with a period of $7 \mathrm{~min}$ was picked up about $100 \mathrm{Mm}$ higher in the corona. It may have been present closer in but there is lots of confusion by overlapping structures below $x=1100 "$ so it is very difficult to see. All oscillations showed a high initial pulse supporting the idea of an impulsive trigger.

Doppler oscillations in Fe XIX of this width and with periods about 14 min are commonly seen at the onset of flares (Wang et al. 2003b). They are generally interpreted as due to slow mode standing waves in hot coronal loops excited at their footpoints because their periods match that expected for the fundamental of the slow mode. This is true for the loops here as well. If we assume a semi-circular loop of height $50 \mathrm{Mm}$ (the height of the slit from the limb) and a sound speed $380 \mathrm{~km} \mathrm{~s}^{-1}$ (Wang et al. 2003b), the fundamental mode would have a period of roughly $14 \mathrm{~min}$. The difference between the events in Wang et al. (2003b) and the event described here is that here there were (i) many, not just one loop, along the line-of-sight, (ii) the trigger seems to be the same as 
that which caused the TRACE oscillation, and (iii) there was a very short (4 min) period Doppler oscillation that cannot be attributed to a slow mode standing wave. Also the large scale of the oscillations suggests that the excitation site was in the corona rather than at a loop footpoint.

In 1-D loops, a coronal trigger would excite the first harmonic and the expected period is half the fundamental (i.e. $7 \mathrm{~min}$ ) which is too short to explain the observed oscillation. However, when a twisted loop erupts, Haynes et al. (2008) demonstrated with simulations of a 3-D loop that the first harmonic of the slow mode rapidly relaxes to a fundamental slow mode wave. Thus the large-scale 14 min oscillations could be the fundamental slow mode triggered by a passing blast wave in the corona. The $4 \mathrm{~min}$ oscillation could then be explained as the Doppler signature of the transverse kink oscillation in loops at the height of the SUMER slit. The 7 min TRACE oscillation was seen at almost twice the height in the corona, and can therefore be explained as the fundamental kink mode in a loop with twice the length.

The re-analysis of the 16 April flare-CME event has revealed three important new features of the plasma flows at the time of the flare and filament eruption, that had been previously interpreted in the context of the CSHKP scenario. All three features indicate that the observed high velocity Fe XIX Doppler shifts $\left(>250 \mathrm{~km} \mathrm{~s}^{-1}\right.$, blue ones reaching a maximum $600 \mathrm{~km} \mathrm{~s}^{-1}$ ) are caused by a blast wave rather than reconnection jets. The features can be summarized as follows.

- Large red and blue Doppler shifts are seen at least 5 minutes ahead of the erupting filament. They lasted longer than 25 minutes, sometimes spreading over 70" $(50000 \mathrm{~km})$ along the SUMER slit. This is incompatible with reconnection jets confined to a narrow region along the current sheet, behind the erupting loop (Fig. 4.12).

- During the filament eruption, a Doppler shift oscillation of the Fe XIX line and an intensity oscillation of $195 \AA$ emission was seen spreading over a region at least twice the size of the area crossed by the filament. Large-scale loop oscillations are known to be excited by blast waves triggered during flares and CME eruptions.

- One of the outlying loops of the active region is seen to suddenly brighten and twist simultaneously along its whole length as though it was hit by a passing wave. The rapid transverse motion of its apex could be also a result of a blast wave interaction with the loop, as in the case of kink oscillations (Hudson \& Warmuth 2004). 


\section{Outlook}

In the presented work, we have studied large scale coronal waves seen in TRACE EUV images and their connection to the loop oscillations excited in their wakes. The oscillations have mostly been seen with the SUMER spectrometer in the Doppler shift of the flare line Fe XIX 1118.05 $\AA$ with the formation temperature above $10 \mathrm{MK}$. Large Doppler shifts (greater than $500 \mathrm{~km} \mathrm{~s}^{-1}$ ) and large-scale Doppler-shift oscillations with periods 3-20 min were found.

Until now, the understanding of the observed events has been often blurred due to the restricted capabilities of the past and present missions. The possibilities which rise with the new missions will help overcome the difficulties related to the data interpretation. STEREO (The Solar Terrestrial Relations Observatory, Kaiser et al. (2008)) is designed to study the three-dimensional evolution of solar eruptions. It consists of two identical spacecrafts orbiting the Sun at nearly 1 AU, with one ahead (STEREO A) and one behind (STEREO B) the Earth near the ecliptic plane. STEREO-EUVI imagers (Wuelser et al. 2004) cover the same emission lines as TRACE $171 \AA$ (1 MK), $195 \AA(1.5 \mathrm{MK})$, $284 \AA(2 \mathrm{MK})$ and $304 \AA(0.08 \mathrm{MK})$.

The AIA (Atmospheric Imaging Assembly) imager onboard of SDO (Solar Dynamics observatory, Rochus et al. (2002)), designed to help understand the influence of the solar activity on Earth, will provide us with images of the solar atmosphere in 10 different wavelengths with a very high temporal ( $\lesssim 10 \mathrm{~s})$ and spatial (1") resolution. It will help us understand the relationship between the solar activity and its drivers.

EIS/HINODE (EUV Imaging Spectrometer, Doschek et al. (2006)) spectroscopic observations provide us with information about the line-of-sight velocity component, which has proved helpful in identifying oscillations as well as flows barely seen in simultaneous EUV imaging data. Understanding of emission processes using SUMER spectrometer have sometimes been complicated by contamination from blends. A careful analysis of the line profiles is necessary in order to differentiate between actual shifts and emission from blends. The EIS spectrometer observes simultaneously in two EUV bands, $170-210 \AA$ and 250-290 $\AA$, covering a large number of emission lines from the transition region, corona, and flares which will help understand the reasons of emissions and dimmings in various lines, and thus give information on the heating and cooling processes. Line profiles, Doppler shifts and Doppler widths, obtained from the EIS 
spectrometer will be analyzed using Wavelet analysis and used to identify waves and oscillations in the corona.

The combination of the new observations from EIS/HINODE, EUVI/STEREO and AIA/SDO offers an unprecedented view on processes in the corona, allowing a better understanding of the geometry and timing, which is important in the attempt to trace the origin of the studied eruptions. 


\section{A Appendix}

\section{A.1 Notation}

\section{A.1.1 Quantities}

\begin{tabular}{|c|c|c|}
\hline symbol & quantity & units \\
\hline$A$ & amplitude of a sound wave & $\mathrm{km} \mathrm{s}^{-1}$ \\
\hline$A_{\mathrm{ul}}$ & Einstein's coefficient for spontaneous emission & $\mathrm{s}^{-1}$ \\
\hline$b$ & impact parameter & $\mathrm{cm}$ \\
\hline $\mathbf{B}$ & magnetic flux density, magnetic induction & $\begin{array}{l}\text { Gauss } \\
1 \mathrm{G}=10^{-4} \mathrm{~T}\end{array}$ \\
\hline $\begin{array}{l}c_{A}(5.2) \\
c_{k}(5.4) \\
c_{s}(5.1) \\
c_{T}\left(\begin{array}{ll}5.3 \\
5.3\end{array}\right.\end{array}$ & $\begin{array}{l}\text { Alfvén } \\
\text { kink speed } \\
\text { sound speed } \\
\text { tube speed }\end{array}$ & $\begin{array}{l}\mathrm{km} \mathrm{s}^{-1} \\
\mathrm{~km} \mathrm{~s}^{-1} \\
\mathrm{~km} \mathrm{~s}^{-1} \\
\mathrm{~km} \mathrm{~s}^{-1}\end{array}$ \\
\hline $\mathbf{E} ; E=|\mathbf{E}|$ & electric field vector; el. field strength & $\mathrm{NC}^{-1}$ \\
\hline $\begin{array}{l}E_{n} \\
E_{u} \\
E_{l}\end{array}$ & $\begin{array}{l}\text { excitation energy of the level } n \\
\text { upper energy level } \\
\text { lower energy level }\end{array}$ & $\begin{array}{l}\mathrm{eV} \\
\mathrm{eV} \\
\mathrm{eV} \\
1 \mathrm{eV}=1,6 \cdot 10^{-19} \mathrm{~J}\end{array}$ \\
\hline$f_{\mathrm{v}} d \mathrm{v}(\mathrm{A} .6)$ & Maxwell velocity distribution & \\
\hline$\overline{\mathcal{F}_{c}}$ & conductive heat flux & 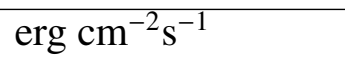 \\
\hline$F_{\text {visc }}$ & viscous force density & $\mathrm{Nm}^{-3}$ \\
\hline $\begin{array}{l}g_{\text {ff }} \\
g_{\text {bf }}\end{array}$ & $\begin{array}{l}\text { free-free Gaunt factor } \\
\text { bound-free Gaunt factor }\end{array}$ & \\
\hline $\begin{array}{l}g_{l} ; g_{u} \\
g_{Z}\end{array}$ & $\begin{array}{l}\text { statistical weight of excitation level } l ; u \\
\text { number of degenerate sub-levels of the level } l ; u \\
\text { statistical weight of ionization state } Z\end{array}$ & \\
\hline $\mathbf{g}$ & gravitational acceleration & $\mathrm{ms}^{-2}$ \\
\hline$G\left(T, \lambda_{\mathrm{lu}}\right)(2.9)$ & contribution function & \\
\hline$G_{v}^{f f}$ & free-free Gaunt factor & \\
\hline
\end{tabular}


A Appendix

\begin{tabular}{|c|c|c|}
\hline symbol & quantity & units \\
\hline $\mathcal{G}(\mathrm{A} .20)$ & Gauss distribution function & \\
\hline $\begin{array}{l}I \\
I_{\mathrm{th}} \\
I_{\mathrm{obs}} \\
I\left(\lambda_{\mathrm{lu}}\right)=I_{\mathrm{lu}}\end{array}$ & $\begin{array}{l}\text { radiance, intensity } \\
\text { theoretically predicted intensity } \\
\text { observed intensity } \\
\text { spectral radiance, specific intensity }\end{array}$ & 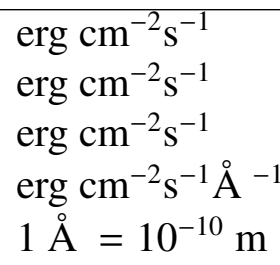 \\
\hline$I_{n} ; K_{n}$ & modified Bessel functions of order $n$ & \\
\hline $\mathbf{j}$ & electric current density & \\
\hline $\begin{array}{l}\mathbf{k}=\left(k_{x}, k_{y}, k_{z}\right) \\
k=|\mathbf{k}|=2 \pi / \lambda\end{array}$ & $\begin{array}{l}\text { wave vector } \\
\text { wave number }\end{array}$ & $\begin{array}{l}\mathrm{cm}^{-1} \\
\mathrm{~cm}^{-1}\end{array}$ \\
\hline$L$ & loop length & $\mathrm{Mm}$ \\
\hline$n$ & principal quantum number & \\
\hline$n_{H}$ & harmonic number & \\
\hline $\mathrm{N}$ & number of elements of the time series & \\
\hline$N$ & particle number density & $\mathrm{cm}^{-3}$ \\
\hline$N_{e}$ & electron number density & $\mathrm{cm}^{-3}$ \\
\hline$N_{\mathrm{H}}$ & proton number density & $\mathrm{cm}^{-3}$ \\
\hline$N_{l}\left(X^{+Z}\right)$ & lower level $(l)$ population of the ion $X^{+Z}$ & $\mathrm{~cm}^{-3}$ \\
\hline$N_{u}\left(X^{+Z}\right)$ & upper level $(u)$ population of the ion $X^{+Z}$ & $\mathrm{~cm}^{-3}$ \\
\hline$N_{Z} ; N_{Z+1}$ & population of charge state $Z ; Z+1$ & $\mathrm{~cm}^{-3}$ \\
\hline$p_{g}(3.3)$ & gas pressure & $\operatorname{erg~\mathrm {cm}^{-3}}$ \\
\hline$p_{m}(3.4)$ & magnetic pressure & $\operatorname{erg~\mathrm {cm}^{-3}}$ \\
\hline $\mathcal{P}$ & period & $\min$ \\
\hline$Q$ & heat & erg \\
\hline$Q_{\text {visc }}$ & viscous losses & 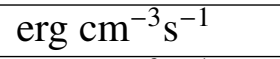 \\
\hline$Q_{\mathrm{rad}}$ & radiative loss function & 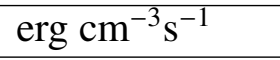 \\
\hline $\mathbf{r}$ & position vector & $\mathrm{m}$ \\
\hline$r_{0}=\frac{1}{4 \pi \epsilon_{0}} \frac{e^{2}}{m c^{2}}$ & classical electron radius & $2.8 \times 10^{-15} \mathrm{~m}$ \\
\hline$s$ & wavelet scale & \\
\hline$\{\mathcal{S}(s, \mathcal{T})\}$ & time series & \\
\hline$t$ & time & $\mathrm{s}$ \\
\hline $\mathcal{T}$ & dimensionless parameter related to time & \\
\hline $\begin{array}{l}T \\
T_{e}\end{array}$ & $\begin{array}{l}\text { temperature } \\
\text { electron temperature }\end{array}$ & $\begin{array}{l}\mathrm{K} \\
\mathrm{K}\end{array}$ \\
\hline$\Delta t$ & time step of the observations & $\mathrm{s}$ \\
\hline $\begin{array}{l}\mathbf{v} ; \mathrm{v}=|\mathbf{v}| \\
\mathbf{v}_{1} \\
\mathrm{v}_{\mathrm{ph}}\end{array}$ & $\begin{array}{l}\text { velocity; speed } \\
\text { velocity perturbation } \\
\text { phase speed }\end{array}$ & $\begin{array}{l}\mathrm{km} \mathrm{s}^{-1} \\
\mathrm{~km} \mathrm{~s}^{-1} \\
\mathrm{~km} \mathrm{~s}^{-1}\end{array}$ \\
\hline$V$ & volume & $\mathrm{cm}^{-3}$ \\
\hline
\end{tabular}


A.1 Notation

\begin{tabular}{|c|c|c|}
\hline symbol & quantity & units \\
\hline $\mathcal{W}(s, \mathcal{T})$ & wavelet coefficient & \\
\hline$W$ & work & erg \\
\hline$E_{n}^{Z}(\mathrm{~A} .14)$ & ionization energy & $\mathrm{eV}$ \\
\hline$E_{\mathrm{ul}}=E_{u}-E_{l}$ & excitation energy & $\mathrm{eV}$ \\
\hline$z$ & distance along the loop or LOS & $\mathrm{cm}$ \\
\hline$Z$ & charge state & \\
\hline $\mathcal{Z}$ & proton number & \\
\hline$\beta \sqrt{3.1)}$ & plasma $\beta$ & \\
\hline$\epsilon_{\lambda}$ & volume emissivity, emission coefficient & $\operatorname{erg~\mathrm {cm}^{-3}}$ \\
\hline$\zeta_{n}$ & incomplete fraction of a shell $n$ & \\
\hline$\eta(3.15)$ & magnetic diffusivity, resistivity & $\mathrm{cm}^{2} \mathrm{~s}^{-1}$ \\
\hline$\kappa$ & thermal conductivity & $\mathrm{s}^{-1}$ \\
\hline$\lambda$ & wavelength & $\AA$ \\
\hline$\Lambda$ & Coulomb logarithm & \\
\hline$\Lambda_{B}$ A.13) & de Broglie wavelength & \\
\hline$\mu$ & magnetic permeability & \\
\hline $\begin{array}{l}v \\
v_{c}(3.17)\end{array}$ & $\begin{array}{l}\text { frequency } \\
\text { frequency of Coulomb collisions }\end{array}$ & $\begin{array}{l}s^{-1} \\
s^{-1}\end{array}$ \\
\hline$v_{\text {visc }}$ & kinematic viscosity & $\mathrm{cm}^{2} \mathrm{~s}^{-1}$ \\
\hline$\omega_{0}$ & dimensionless parameter related to frequency & \\
\hline$\Theta=(\mathbf{B} \cdot \mathbf{k}) /(|\mathbf{B}| \cdot|\mathbf{k}|)$ & angle between $\mathbf{B}_{\mathbf{0}}$ and $\mathbf{k}$ & $\mathrm{rad}$ \\
\hline$\rho$ & mass density & $\mathrm{kgcm}^{-3}$ \\
\hline$\sigma(3.16)$ & electric conductivity & $\mathrm{AV}^{-1} \mathrm{~cm}^{-1}$ \\
\hline$\sigma_{Q}$ & charge density & $\mathrm{Ccm}^{-2}$ \\
\hline $\begin{array}{l}\sigma_{v}{ }^{b f} \\
\sigma_{v}{ }^{f b} \\
\sigma_{X^{+Z}}\end{array}$ & $\begin{array}{l}\text { photo-ionization cross-Section } \\
\text { recombination cross-Section } \\
\text { photo-ionization cross-Section of the ion } \mathrm{X}^{+Z}\end{array}$ & $\begin{array}{l}\mathrm{cm}^{-2} \\
\mathrm{~cm}^{-2} \\
\mathrm{~cm}^{-2}\end{array}$ \\
\hline$\tau$ & optical thickness & \\
\hline$\phi$ & spectral distribution function & \\
\hline $\begin{array}{l}\omega=2 \pi v \\
\omega_{\mathcal{T}}(6.4)\end{array}$ & $\begin{array}{l}\text { circular frequency } \\
\text { discrete circular frequency }\end{array}$ & $\begin{array}{l}\mathrm{Hz} \\
\mathrm{Hz}\end{array}$ \\
\hline
\end{tabular}


A Appendix

\section{A.1.2 Constants}

\begin{tabular}{|l|l|l|}
\hline symbol & constant & approx. value and units \\
\hline$c$ & speed of light & $2,99 \times 10^{8} \mathrm{~km} \mathrm{~s}^{-1}$ \\
\hline$e$ & electron charge & $-1,6 \cdot 10^{-19} \mathrm{C}$ \\
\hline $\mathbf{g}_{\mathbf{0}}$ & gravitational acceleration & $2.74 \times 10^{2} \mathrm{~ms}^{-2}$ \\
\hline & near the solar surface & \\
\hline$h$ & Planck constant & $6.62610^{-41} \mathrm{erg} \mathrm{s}$ \\
\hline$h_{\text {low }} \sqrt[6.5]{ }$ & discrete low pass filter & \\
\hline$k_{B}$ & Boltzmann constant & $1.38 \times 10^{-23} \mathrm{ergK}^{-1}$ \\
\hline$m_{e}$ & electron mass & $9,1 \cdot 10^{-31} \mathrm{~kg}^{-15}$ \\
\hline$r_{0}=\frac{1}{4 \pi \epsilon_{0}} \frac{e^{2}}{m c^{2}}$ & classical electron radius & $2.8 \times 10^{-15} \mathrm{~m}$ \\
\hline$\epsilon_{0}$ & permittivity of vacuum, electric constant & $8,854 \mathrm{Fcm}^{-1}$ \\
\hline$\mu_{0}$ & permeability of free space & 1 \\
\hline$\sigma_{T}=(8 / 3) \pi r_{0}^{2}$ & Thompson cross-Section & $6.65 \times 10^{-31} \mathrm{~cm}^{2}$ \\
\hline$\Psi\left(s, \omega_{\mathcal{T}}\right)$ & scaled and translated mother wavelet & \\
\hline
\end{tabular}




\section{A.2 Spectral Notation}

\section{Electron configuration}

$$
n l^{N}
$$

$n$ principal quantum number

$l=0,1,2,3,4,5, \ldots$ orbital momentum

$s, p, d, f, g, h, \ldots$

$s=1 / 2$ electron spin

Ionic state - Spin-orbital (L-S) coupling

$$
{ }^{2 S+1} L_{J}
$$

$S=\sum \mathbf{s}=$ vector sum of all electron spins

$2 S+1=$ multiplicity $=$ number of possible values of $\mathbf{J}$

$L=\sum \mathbf{l}=0,1,2,3,4,5, \ldots$ vector sum of all electron orbital angular momenta $S, P, D, F, G, H$

$J=L+S$ total angular momentum 
A Appendix

A.3 Line List

\section{A.3.1 SUMER Range}

\begin{tabular}{|c|c|c|c|c|}
\hline Ion & $\begin{array}{c}\lambda \\
{[\AA]}\end{array}$ & Levels u - I & $\log \mathbf{T}$ & $\begin{array}{c}\mathbf{I} \\
{\left[\mathrm{erg} \mathbf{c m}^{-2} \mathbf{s r}^{-1} \mathbf{s}^{-1}\right]}\end{array}$ \\
\hline $\mathrm{C}_{\mathrm{I}}$ & 1112.22 & $2 s^{2} 2 p^{2}{ }^{3} P_{0}-2 s^{2} 2 p 1 d^{1} D_{1}$ & $<4$ & \\
\hline $\mathrm{C}_{\mathrm{I}}$ & 1112.47 & $2 s^{2} 2 p^{2}{ }^{3} P_{1}-2 s^{2} 2 p 1 d^{3} D_{1}$ & $<4$ & \\
\hline $\mathrm{C}_{\mathrm{I}}$ & 1112.80 & $2 s^{2} 2 p^{2}{ }^{3} P_{2}-2 s^{2} 2 p 1 d^{3} D_{1}$ & $<4$ & \\
\hline $\mathrm{C}_{\mathrm{I}}$ & 1112.82 & $2 s^{2} 2 p^{2}{ }^{3} P_{2}-2 s^{2} 2 p 1 d^{3} F_{3}$ & $<4$ & \\
\hline $\mathrm{S}_{\text {III }}$ & 1113.15 & & & \\
\hline $\mathrm{S}_{\text {III }}$ & 1113.23 & $3 s 3 p^{3} P_{2}-3 s 3 d^{3} D_{3}$ & 4.7 & \\
\hline Si III & 1113.1760 & $3 s 3 p^{3} P_{2}-3 s 3 d^{3} D_{1}$ & 4.7 & $4.05 \mathrm{e}+02$ \\
\hline Si III & 1113.2061 & $3 s 3 p^{3} P_{2}-3 s 3 d^{3} D_{2}$ & 4.7 & $5.84 \mathrm{e}+03$ \\
\hline Si III & 1113.2321 & $3 s 3 p^{3} P_{2}-3 s 3 d^{3} D_{3}$ & 4.7 & $4.00 \mathrm{e}+04$ \\
\hline $\mathrm{C}_{\mathrm{I}}$ & 1114.00 & $2 s^{2} 2 p^{2}{ }^{3} P_{1}-2 s^{2} 2 p 1 d^{1} P_{1}$ & $<4$ & \\
\hline $\mathrm{C}_{\mathrm{I}}$ & 1114.39 & & $<4$ & \\
\hline $\mathrm{C}_{\mathrm{I}}$ & 1114.64 & & $<4$ & \\
\hline $\mathrm{C}_{\mathrm{I}}$ & 1114.86 & & $<4$ & \\
\hline $\mathrm{C}_{\mathrm{I}}$ & 1115.17 & & $<4$ & \\
\hline $\mathrm{C}_{\mathrm{I}}$ & 1115.21 & & $<4$ & \\
\hline $\mathrm{Cax}$ & $1115.53 / 2$ & $3 s^{2} S_{1 / 2}-3 p^{2} P_{3 / 2}$ & 5.8 & $1.73 \mathrm{e}+02$ \\
\hline $\mathrm{C}_{\mathrm{I}}$ & 1117.20 & & $<4$ & \\
\hline $\mathrm{Ne}$ VII & $1117.22 / 2$ & $2 s 2 p^{3} P_{1}-2 p^{2}{ }^{3} P_{2}$ & 5.7 & $2.60 \mathrm{e}+02$ \\
\hline $\mathrm{Ne}$ VI & $1117.37 / 2$ & $2 s^{2} 2 p^{2} P_{1 / 2}-2 s 2 p^{2}{ }^{2} D_{3 / 2}$ & 5.6 & $5.57 e+03$ \\
\hline $\mathrm{C}_{\mathrm{I}}$ & 1117.58 & & $<4$ & \\
\hline $\mathrm{C}_{\mathrm{I}}$ & 1117.86 & & $<4$ & \\
\hline $\mathrm{P} v$ & 1117.9790 & $3 s^{2} S_{1 / 2}-3 p^{2} P_{3 / 2}$ & 5.1 & $2.77 \mathrm{e}+02$ \\
\hline Fe XIX & 1118.0575 & $2 s^{2} 2 p^{4{ }^{3}} P_{2}-2 s^{2} 2 p^{4{ }^{3}} P_{1}$ & 7.0 & $3.62 \mathrm{e}+04$ \\
\hline $\mathrm{C}_{\mathrm{I}}$ & 1118.18 & & $<4$ & \\
\hline $\mathrm{C}_{\mathrm{I}}$ & 1118.49 & & $<4$ & \\
\hline Si v & 1118.81 & $2 p^{5} 3 s^{3} P_{2}-2 p^{5} 3 s^{3} P_{2}$ & 5.5 & $1.01 \mathrm{e}+01$ \\
\hline
\end{tabular}


A.3 Line List

\section{A.3.2 TRACE Range}

\begin{tabular}{|c|c|c|c|c|}
\hline Ion & $\begin{array}{c}\lambda \\
{[\AA \mathbf{A}]}\end{array}$ & Levels u - I & $\log \mathbf{~ T}$ & $\begin{array}{c}\text { I } \\
{\left[\mathbf{e r g ~ c m}^{-2} \mathbf{s r}^{-1} \mathbf{s}^{-1}\right]}\end{array}$ \\
\hline Fe XXIV & 192.0285 & $1 s^{2} 2 s^{2} S_{1 / 2}-1 s^{2} 2 p^{2} P_{3 / 2}$ & 7.2 & $1.21 \mathrm{e}+06$ \\
\hline $\mathrm{O}_{\mathrm{v}}$ & 192.7500 & $2 s 2 p^{3} P_{0}-2 s 3 d^{3} D_{1}$ & 5.4 & $1.00 \mathrm{e}+03$ \\
\hline $\mathrm{O}_{\mathrm{v}}$ & 192.7970 & $2 s 2 p^{3} P_{1}-2 s 3 d^{3} D_{2}$ & 5.4 & $2.01 \mathrm{e}+03$ \\
\hline $\mathrm{O}_{\mathrm{v}}$ & 192.7500 & $2 s 2 p^{3} P_{1}-2 s 3 d^{3} D_{1}$ & 5.4 & $7.53 \mathrm{e}+02$ \\
\hline Fe XII & 195.1190 & $3 s^{2} 3 p^{3}{ }^{4} S_{3 / 2}-3 s^{2} 3 p^{2}\left({ }^{3} P\right) 3 d^{4} P_{5 / 2}$ & 6.2 & $1.07 \mathrm{e}+04$ \\
\hline Fe XII & 195.1790 & $3 s^{2} 3 p^{3}{ }^{2} D_{3 / 2}-3 s^{2} 3 p^{2}\left({ }^{1} D\right) 3 d^{2} D_{3 / 2}$ & 6.2 & $7.40 \mathrm{e}+02$ \\
\hline
\end{tabular}




\section{A.4 Radiative Transfer}

Equation of radiative transfer describes the change in specific intensity $d I_{\lambda}$ along a distance $d z$ into a unit solid angle in a medium with opacity given by $\kappa_{\lambda}$ measured in $\left[\mathrm{cm}^{-1}\right]$ :

$$
d I_{\lambda}=-\kappa_{\lambda} I_{\lambda} d z+\epsilon_{\lambda} d z \quad\left[\operatorname{erg~cm}{ }^{-2} \mathrm{~s}^{-1} \mathrm{ster}^{-1}\right],
$$

with a formal solution

$$
I_{\nu}\left(\tau_{v}\right)=I_{v}(0) e^{-\tau_{v}}+\int_{0}^{\tau_{v}} S\left(\tau_{v}^{\prime}\right) e^{-\left(\tau_{\nu}-\tau_{v}^{\prime}\right)} d \tau_{v}^{\prime},
$$

where $\tau_{v}$ is a dimensionless parameter called optical thickness of the medium,

$$
d \tau_{v}=-\kappa_{v} d z
$$

and $S_{\lambda}=\epsilon_{\lambda} / \kappa_{\lambda}$ is the source function of the radiation field, which in LTE case equals Planck function A.15. 


\section{A.5 Local Thermodynamic Equilibrium}

Under the LTE conditions, all particles have the shape of a Maxwellian distribution, ionization is described with the Saha equation, excitation by Boltzmann equation, and radiation is given by the Panck function.

\section{A.5.1 Maxwellian Distribution}

Velocity distribution function of electrons and ions in a thermal plasma is:

$$
f_{\mathbf{v}}(\mathbf{v}) d \mathbf{v}=\sqrt{\frac{2}{\pi}}\left(\frac{\mathrm{m}}{\mathrm{k}_{\mathrm{B}} \mathrm{T}}\right)^{3 / 2} \mathrm{v}^{2} \exp \left[-\frac{m \mathrm{v}^{2}}{2 \mathrm{k}_{\mathrm{B}} \mathrm{T}}\right] \mathrm{dv}
$$

It is normalized,

$$
\int_{0}^{\infty} f_{\mathbf{v}}(\mathbf{v}) d \mathbf{v}=1
$$

with a distribution parameter:

$$
a=\sqrt{k_{B} T / m}
$$

The most probable value $\mathrm{v}_{\mathrm{p}}$ is the maximum value in curves in Fig. A.1, i.e. the solution of $d f_{\mathrm{v}} / d \mathrm{v}=0$ :

$$
\mathrm{v}_{\mathrm{p}}=\sqrt{2 k_{B} T / m}
$$

Mean value:

$$
<\mathrm{v}>=\int_{0}^{\infty} \mathrm{f}_{\mathrm{v}} \mathrm{vdv}=2 \sqrt{2 k_{B} T / \pi m}
$$

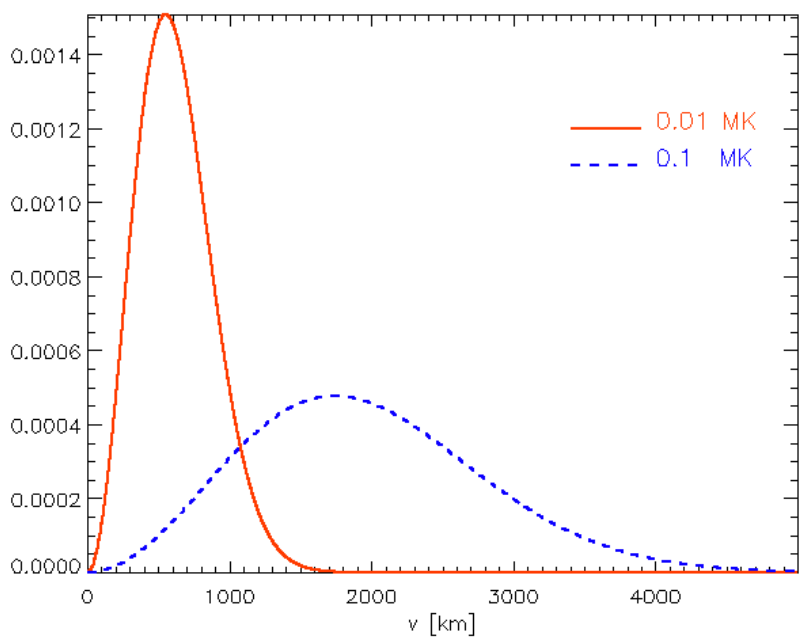

Figure A.1: Maxwellian electron distribution for 2 different temperatures. 


\section{A.5.2 Boltzmann equation}

Under the assumption of LTE, the population density of a state $u$ with a statistical weight $g_{u}$ and energy levels $E_{u}$ is given by Boltzmann equation:

$$
\frac{N_{u}}{N}=\frac{g_{u} \exp \left[-\frac{\left(E_{u}\right)}{k_{B} T}\right]}{\sum g_{n} \exp \left[-E_{n} / k_{B} T\right]} .
$$

The sum in the expression of partition function $\sum g_{n} \exp \left[-E_{n} / k_{B} T\right]$ goes over all possible excitation states of the ion.

\section{A.5.3 Saha Ionization Equation}

Degree of ionization of plasma in local thermodynamic equilibrium (LTE) is a function of electron temperature and density and ionization energies of the atoms it contents:

$$
\frac{N_{Z+1} N_{e}}{N_{Z}}=\frac{2}{\Lambda^{3}} \frac{\sum g_{n, Z+1} \exp \left[-E_{n} / k_{B} T\right]}{\sum g_{n, Z} \exp \left[-E_{n} / k_{B} T\right]} \exp \left[-\frac{\left(X^{Z+1}-X^{Z}\right)}{k_{B} T}\right],
$$

where $\Lambda$ is the thermal de Broglie wavelength of an electron

$$
\Lambda \stackrel{\text { def }}{=} \sqrt{\frac{h^{2}}{2 \pi m_{e} k_{B} T}}
$$

Ionization energy $X^{Z}$ is the minimum energy required to remove an electron from a neutral atom in its ground state $(n=1)$. Ionization energy of an atom in a state characterized by the principal quantum number $n$ is

$$
E_{n}^{Z}=-\frac{Z^{2}}{n^{2}} \cdot 13.6 \quad[\mathrm{eV}]
$$

\section{A.5.4 Planck Function}

Power emitted per unit area of emitting surface of a perfect black body, per unit solid angle and frequency, or specific intensity is $\mathcal{U}(v, T)$ : Under the assumption of LTE, ratio of two excited states of an ion $l$ and $u$ with statistical weights $g_{l}, g_{u}$ and energy levels $E_{l}$ and $E_{u}$ is given by Boltzmann equation:

$$
\mathcal{U}(v, T)=\frac{4 \pi}{c} I(v, T)=\frac{8 \pi h v^{3}}{c^{3}} \frac{1}{e^{\frac{h v}{k_{B} T}}-1} \quad\left[\operatorname{erg~cm}^{-2} \operatorname{ster}^{-1} \AA-1\right],
$$

with the maximum power radiated in the wavelength given by Wien's displacement law:

$$
\lambda_{\max }=\frac{2.89 \times 10^{-3}}{T} \quad[\mathrm{~m}]
$$




\section{A.6 Fourier Transform}

A Fourier transform of a signal represented by a time series $\mathcal{S}(\mathcal{T})$ is its representation in the frequency domain given by:

$$
\hat{\mathcal{S}}\left(\omega_{0}\right)=\sum_{-\infty}^{\infty} \mathcal{S}(\mathcal{T}) e^{-2 \pi i \mathcal{T} \omega_{0}} .
$$

The series $\mathcal{S}(\mathcal{T})$ can be reconstructed by the inverse transform:

$$
\mathcal{S}(\mathcal{T})=\sum_{-\infty}^{\infty} \hat{\mathcal{S}}\left(\omega_{0}\right) e^{2 \pi i \mathcal{T} \omega_{0}} d \omega_{0}
$$

A short-time Fourier transform (STFT) or windowed Fourier transform defined by Gabor (1946) is:

$$
\operatorname{STFT}\left(\mathcal{S}\left\{\mathcal{T}, \omega_{0}\right\}\right)=\sum_{-\infty}^{\infty} \mathcal{S}(\mathcal{T}) \mathcal{G}\left(\mathcal{T}^{\prime}-\mathcal{T}\right) e^{-i \omega_{0} \mathcal{T}^{\prime}}
$$

where

$$
\mathcal{G}\left(\mathcal{T}^{\prime}-\mathcal{T}\right)=\frac{1}{\sigma \sqrt{2 \pi}} e^{-\frac{\left(\mathcal{T}^{\prime}-\mathcal{T}\right)^{2}}{2 \sigma^{2}}}
$$

is a Gaussian window and $\sigma$ is its standard deviation.

Convolution theorem states that the Fourier transform of a convolution of 2 functions is a product of their respective Fourier transforms

$$
F T\left(\mathcal{S} * \Psi^{*}\right)=F T(\mathcal{S}) \cdot F T\left(\Psi^{*}\right)
$$





\section{Bibliography}

Amari, T., Luciani, J. F., Aly, J. J., \& Tagger, M. 1996, ApJ, 466, 39

Amari, T., Luciani, J. F., Mikic, Z., \& Linker, J. 2000, ApJ, 529, 49

Antiochos, S. K., DeVore, C. R., \& Klimchuk, J. A. 1999, ApJ, 510, 485

Anzer, U. \& Heinzel, P. 2005, ApJ, 622, 714

Aschwanden, M. J. 2004, in ESA SP-575: SOHO 15 Coronal Heating, ed. R. W. Walsh, J. Ireland, D. Danesy, \& B. Fleck, 97

Aschwanden, M. J., de Pontieu, B., Schrijver, C. J., \& Title, A. M. 2002, Sol. Phys., 206, 99

Aschwanden, M. J., Fletcher, L., Schrijver, C. J., \& Alexander, D. 1999, ApJ, 520, 880

Aschwanden, M. J., Wuelser, J. P., Nitta, N. V., \& Lemen, J. R. 2009, Sol. Phys., 256, 3

Aulanier, G., DeLuca, E. E., Antiochos, S. K., McMullen, R. A., \& Golub, L. 2000, ApJ, 540, 1126

Beigman, I. L., Vainshtein, L. A., \& Vinogradov, A. 1970, Soviet Astronomy, 13, 775

Bryans, P., Landi, E., \& Savin, D. W. 2009, ApJ, 691, 1540

Carmichael, H. 1964, NASA Special Publication, 50, 451

Chen, J., Howard, R. A., Brueckner, G. E., et al. 1997, ApJ, 490, 191

Cheng, C. C. \& Rosenberg, F. D. 1976, in Bulletin of the American Astronomical Society, Vol. 8, Bulletin of the American Astronomical Society, 556

Choe, G. S. \& Lee, L. C. 1996, ApJ, 472, 372

Cowling, T. G. 1953, Solar Electrodynamics, 532

Cowling, T. G. 1976, Magnetohydrodynamics, ed. Cowling, T. G. 
Curdt, W., Brekke, P., Feldman, U., et al. 2001, A\&A, 375, 591

Curdt, W., Landi, E., \& Feldman, U. 2004, A\&A, 427, 1045

Dennis, B. R., Chupp, E. L., Crannel, C. J., \& co authors, . 1986, NASA STI/Recon Technical Report N, 87, 13379

Dennis, B. R. \& Schwartz, R. A. 1989, Sol. Phys., 121, 75

Dere, K. P., Brueckner, G. E., Howard, R. A., Michels, D. J., \& Delaboudiniere, J. P. 1999, ApJ, 516, 465

Doschek, G. A., Brown, C. M., Korendyke, C. M., et al. 2006, in Bulletin of the American Astronomical Society, Vol. 38, Bulletin of the American Astronomical Society, 260

Doschek, G. A., Dere, K. P., Sandlin, G. D., et al. 1975, ApJ, 196, 83

Dryer, M. 1982, Space Science Reviews, 33, 233

Dungey, J. W. 1953, MNRAS, 113, 679

Edwin, P. M. \& Roberts, B. 1983, Sol. Phys., 88, 179

Erdélyi, R. \& Fedun, V. 2007, Science, 318, 1572

Feldman, U., Mandelbaum, P., Seely, J. F., Doschek, G. A., \& Gursky, H. 1992, ApJ, 81,387

Fontenla, J. M., Avrett, E. H., \& Loeser, R. 1990, ApJ, 355, 700

Forbes, T. G. 1991, Geophysical and Astrophysical Fluid Dynamics, 62, 15

Gabor, D. 1946, J.IEE, 93, 429

Gabriel, A. H. 1976, Royal Society of London Philosophical Transactions Series A, 281,339

Gary, G. A. \& Moore, R. L. 2004, ApJ, 611, 545

Giovanelli, R. G. 1946, Nature, 158, 81

Giovanelli, R. G. 1948, MNRAS, 108, 163

Goff, C. P., van Driel-Gesztelyi, L., Harra, L. K., Matthews, S. A., \& Mandrini, C. H. 2005, A\&A, 434, 761 
Gold, T. 1964, NASA Special Publication, 50, 389

Gosling, J. T., Hildner, E., MacQueen, R. M., et al. 1974, J. Geophys. Res., 79, 4581

Griem, H. R. 1969, ApJ, 156, 103

Gronenschild, E. H. B. M. \& Mewe, R. 1978, A\&A, 32, 283

Hanaoka, Y. 1994, in Proceedings of Kofu Symposium, 181-184

Handy, B. N., Acton, L. W., Kankelborg, C. C., et al. 1999, Sol. Phys., 187, 229

Haynes, M., Arber, T. D., \& Verwichte, E. 2008, A\&A, 479, 235

Hirayama, T. 1974, Sol. Phys., 34, 323

Hoyng, P., Duijveman, A., Machado, M. E., et al. 1981, ApJ, 246, 155

Hudson, H. \& Ryan, J. 1995, ARA\&A, 33, 239

Hudson, H. S. \& Warmuth, A. 2004, ApJ, 614, 85

Innes, D., McIntosh, S., \& Pietarila, A. 2010, ArXiv e-prints

Kahler, S. W. 1992, ARA\&A, 30, 113

Kaiser, M. L., Kucera, T. A., Davila, J. M., et al. 2008, Space Sci. Rev., 136, 5

Karzas, W. J. \& Latter, R. 1961, ApJ, 6, 167

Kliem, B., Dammasch, I. E., Curdt, W., \& Wilhelm, K. 2002, ApJ, 568, 61

Klimchuk, J. A. 2001, Space Weather (Geophysical Monograph 125), ed. P. Song, H. Singer, G. Siscoe (Washington: Am. Geophys. Un.), 143 (2001), 125, 143

Kopp, R. A. \& Pneuman, G. W. 1976, Sol. Phys., 50, 85

Krucker, S., Battaglia, M., Cargill, P. J., et al. 2008, A\&A Rev., 16, 155

Kuperus, M. \& Raadu, M. A. 1974, A\&A, 31, 189

Leroy, J. L., Bommier, V., \& Sahal-Brechot, S. 1983, Sol. Phys., 83, 135

Leroy, J. L., Bommier, V., \& Sahal-Brechot, S. 1984, A\&A, 131, 33

Lin, J. \& Forbes, T. G. 2000, J. Geophys. Res., 105, 2375

Linker, J. A. \& Mikic, Z. 1995, ApJ, 438, 45 
Mackay, D. H., Karpen, J. T., Ballester, J. L., Schmieder, B., \& Aulanier, G. 2010, Space Sci. Rev., 151, 333

Malin, D. F. 1977, in AAS Photo Bulletin, Vol. 16, AAS Photo Bulletin, 10-13

Manoharan, P. K. \& Kundu, M. R. 2003, ApJ, 592, 597

Masuda, S., Kosugi, T., Hara, H., Tsuneta, S., \& Ogawara, Y. 1994, Nature, 371, 495

Mazzotta, P., Mazzitelli, G., Colafrancesco, S., \& Vittorio, N. 1998, A\&A, 133, 403

McKenzie, J. F. 1970, J. Geophys. Res., 75, 5331

Mikic, Z. \& Linker, J. A. 1994, ApJ, 430, 898

Nakariakov, V. M., Ofman, L., Deluca, E. E., Roberts, B., \& Davila, J. M. 1999, Science, 285,862

Nelson, G. J. \& Melrose, D. B. 1985, Type II bursts, 333-359

Ofman, L. \& Wang, T. 2002, ApJ, 580, 85

Orrall, F. Q. \& Schmahl, E. J. 1976, Sol. Phys., 50, 365

Parker, E. N. 1963, ApJ, 8, 177

Parker, E. N. 1973, ApJ, 180, 247

Petschek, H. E. 1964, NASA Special Publication, 50, 425

Poedts, S., Goossens, M., \& Kerner, W. 1989, Sol. Phys., 123, 83

Roberts, B. 1981, Sol. Phys., 69, 27

Roberts, B. 1983, Sol. Phys., 87, 77

Rochus, P., Defise, J. M., Halain, J. P., et al. 2002, AGU Fall Meeting Abstracts

Schrijver, C. J., Aschwanden, M. J., \& Title, A. M. 2002, Sol. Phys., 206, 69

Spitzer, Jr., L. \& Greenstein, J. L. 1951, ApJ, 114, 407

Spruit, H. C. 1982, Sol. Phys., 75, 3

Stenborg, G. \& Cobelli, P. J. 2003, A\&A, 398, 1185

Stenborg, G., Vourlidas, A., \& Howard, R. A. 2008, ApJ, 674, 1201 
Sterling, A. C. \& Moore, R. L. 2001, AGU Spring Meeting Abstracts, 51

Sterling, A. C. \& Moore, R. L. 2004, ApJ, 602, 1024

Sterling, A. C., Moore, R. L., Qiu, J., \& Wang, H. 2001, ApJ, 561, 1116

Sturrock, P. A. 1966, Nature, 211, 695

Sturrock, P. A. 1968, in IAU Symp. 35, Structure and Development of Solar Active Regions, ed. K. O. Kiepenheuer (Dordrecht: Reidel), 471

Subramanian, P., Ananthakrishnan, S., Janardhan, P., et al. 2003, Sol. Phys., 218, 247

Sui, L. \& Holman, G. D. 2003, ApJ, 596, 251

Sui, L., Holman, G. D., \& Dennis, B. R. 2004, ApJ, 612, 546

Sweet, P. A. 1958, in IAU Symposium, Vol. 6, Electromagnetic Phenomena in Cosmical Physics, ed. B. Lehnert, 123

Sweet, P. A. 1969, ARA\&A, 7, 149

Tsiklauri, D., Nakariakov, V. M., Arber, T. D., \& Aschwanden, M. J. 2004, A\&A, 422, 351

Tucker, W. H. \& Gould, R. J. 1966, ApJ, 144, 244

Tucker, W. H. \& Koren, M. 1971, ApJ, 168, 283

Wang, H., Qiu, J., Jing, J., \& Zhang, H. 2003a, ApJ, 593, 564

Wang, T., Solanki, S. K., Curdt, W., Innes, D. E., \& Dammasch, I. E. 2002, ApJ, 574, 101

Wang, T., Sui, L., \& Qiu, J. 2007, ApJ, 661, 207

Wang, T. J. \& Solanki, S. K. 2004, A\&A, 421, 33

Wang, T. J., Solanki, S. K., Curdt, W., et al. 2003b, å, 406, 1105

Wang, T. J., Solanki, S. K., Innes, D. E., \& Curdt, W. 2005, å, 435, 753

Wild, J. P. 1950, Australian Journal of Scientific Research A Physical Sciences, 3, 541

Wilhelm, K., Curdt, W., Marsch, E., et al. 1995, Sol. Phys., 162, 189

Wu, S. T. 1982, Space Science Reviews, 32, 115 
Wuelser, J., Lemen, J. R., Tarbell, T. D., et al. 2004, in Presented at the Society of Photo-Optical Instrumentation Engineers (SPIE) Conference, Vol. 5171, Society of Photo-Optical Instrumentation Engineers (SPIE) Conference Series, ed. S. Fineschi \& M. A. Gummin, 111-122

Young, P. R., Del Zanna, G., Mason, H. E., et al. 2007, PASJ, 59, 727

Zhang, J., Dere, K., \& Howard, R. A. 2001, AGU Fall Meeting Abstracts

Zhugzhda, Y. D. \& Nakariakov, V. M. 1999, Physics Letters A, 252, 222 


\section{Publications}

\section{Publications in refereed scientific journals}

- Dzifcáková, E. and Tóthová, D., "Synthetic Spectra of the Fe vIII - Fe Xvi Emission Lines for Electron Non-Thermal Distributions", Solar Physics, Volume 240, Issue 4, p. $211-226$

- Tóthová, D., Innes D. E. and Stenborg G., "Oscillations in the wake of a flare blast wave", Letter to editor, Astronomy $\mathcal{E}$ Astrphysics, A $\mathcal{E} A$, Volume 528, p.12

\section{Conference proceedings}

- Tóthová, D., Innes D. E., "High Speed Plasma Acceleration and Loop Oscillations at Flare Onset", 12th European Solar Physics Meeting, Freiburg, Germany, held September, 8-12, 2008.

- Tothova, D.; Innes, D. E.; Solanki, S. K., "Wavelet-based method for coronal loop oscillations analysis", Modern solar facilities - advanced solar science, Proceedings of a Workshop held at Göttingen September 27-29

- Tothova, D.; Innes, D. E.; Solanki, S. K., "Microflares and Hot Coronal Loop Oscillations", SOHO-17. 10 Years of SOHO and Beyond, Proceedings of the conference held 7-12 May, 2006 at Giardini Naxos, Sicily, Italy.

- Tóthová, D. and Dzifcáková, E., "The Influence of the Electron Power Distribution on the Excitation Equilibrium of the Ions in the Solar Corona", 2006,SOHO17. 10 Years of SOHO and Beyond, Proceedings of the conference held 7-12 May, 2006 at Giardini Naxos, Sicily, Italy. 



\section{Acknowledgements}

I would like to thank the Max Planck Institute for Solar System Research and the International Max Planck Research School for the opportunity and the financial support.

My special thanks goes to Dr. Davina Innes for her help throughout my stay at the research school, for her patience, encouragements and comments on my work which I very much appreciate.

I thank my supervisors, Prof. Sami Solanki and Prof. Franz Kneer, and the coordinator of the research school Dr. Dieter Schmitt, for the opportunity I was offered, and for all their support and valuable comments.

I would like to thank Angelos Vourlidas for the discussions and Guillermo Stenborg for his good-will when I needed help with the enhancement of the images.

I'm very grateful to my parents for always being there for me and for giving me a chance to study.

Finally, I want to give my thanks to my sister and to all my friends all around the world as well as my colleagues in Lindau for their moral support. 



\title{
Curriculum Vitae
}

\author{
Name Danica Tóthová \\ Geburt 19. Februar 1979 \\ in Bratislava
}

Staatsbürgerschaft: Slowakei

$\begin{array}{lll}\text { Grundschule: } & 09 / 1985-06 / 1986 & \begin{array}{l}\text { Bierutova } 19 \\ \text { Bratislava } \\ \text { Gessayova } 2 \\ \text { Bratislava }\end{array} \\ & 09 / 1986-06 / 1993 & \begin{array}{l}\text { Bilinguales Gymnasium Metodova } \\ \text { Schulbildung: } \quad 09 / 1993-06 / 1998\end{array} \\ & 09 / 1998-06 / 2004 & \begin{array}{l}\text { Physik Diplom Studium, Comenius Universität } \\ \text { Bratislava } \\ \text { Studium: }\end{array} \\ & 02 / 2006-10 / 2010 & \begin{array}{l}\text { Promotion, Max Planck Institut } \\ \text { für Sonnensystemforschung Katlenburg-Lindau }\end{array}\end{array}$ 